\title{
Konzepte für eine sprachbewusste Hochschullehre
}

\author{
Inhaltsverzeichnis
}

2.1 Sprache im Fach erleben - 55

2.1.1 Sprache in Spielsituationen - 56

2.1.2 Sprachbeobachtungen im fachlichen Spiel - 58

2.2 Aufgaben sprachbewusst planen - 63

2.2.1 Aufgabenvariationen mit mehr "Leichtigkeit" - 63

2.2.2 Sprachbewusste Aufgabenplanung in der Hochschullehre am Beispiel Mittelwerte - 66

2.3 Darstellungsvernetzungen adressieren - 73

2.3.1 Musiker für Schulfest gesucht - Bedingte Wahrscheinlichkeiten - 74

2.3.2 Merkmale des stochastischen Kontexts „Bedingte Wahrscheinlichkeiten" - 75

2.3.3 Sprachbewusste Aufgabenvariation durch Darstellungsvernetzung - 76

2.4 Empirische Daten aus dem Unterricht als Analyse- und Lerngegenstand - 83

2.4.1 Schriftliche und mündliche Unterrichtsdaten in universitären Veranstaltungen - 84

2.4.2 Unterrichtsdiskurs zum Abhängigkeitsbegriff als Lerngegenstand - 85

2.4.3 Schülertext zum Thema "Wahrscheinlichkeiten" als Lerngegenstand -88 


\subsection{Sprache und Fach erkunden durch forschendes}

Lernen - 93

2.5.1 Forschendes Lernen im Praxissemester - 93

2.5.2 Verzahnung der Lernorte Schule und Universität - 95

2.5.3 Stiftung von Kohärenz beim forschenden Lernen - 96

2.5.4 Begleitung der studentischen Projekte im Rahmen der Hochschullehre - 98

2.6 Forschungsfragen entwickeln - 107

2.6.1 Vorläufige Forschungsfragen weiterentwickeln - 108

2.6.2 Kriterien für Forschungsfragen im Bereich Sprachbildung im Fach - 109

2.6.3 Entwicklung von Forschungsfragen - 110 
Im vorliegenden Kapitel werden die Leitideen und hochschuldidaktischen DesignPrinzipien aus Kap. 1 aufgegriffen und anhand von Beispielen für eine sprachbewusste und forschungsbezogene Lehre in der Lehramtsausbildung Mathematik konkretisiert.

In Abschn. 2.1 wird zunächst eine Spielsituation vorgestellt, in der die Studierenden den Kontext „Sprache im Fach Mathematik“ bewusst erleben können. Die Spielsituation bietet einen anschaulichen Einstieg und ermöglicht fach- und sprachbezogene Theoriebildungen ebenso wie eine Annäherung an die Arbeit mit empirischen Daten.

Ein für den Mathematikunterricht - und damit für die Lehramtsausbildung Mathematik - zentraler Aspekt ist die Arbeit mit und die Variation von Aufgaben. In $>$ Abschn. 2.2 steht daher die Frage im Mittelpunkt, wie Studierende bei der sprachbewussten Aufgabenplanung bzw. -variation systematisch unterstützt werden können. Abschn. 2.3 vertieft das Thema mit einem Schwerpunkt auf Darstellungsvernetzung. Es wird aufgezeigt, wie sich dieses wirkungsvolle Mittel zur fachlichen und sprachlichen Bildung in der Hochschullehre und insbesondere mit Blick auf studentische Erprobungen in Praxisphasen thematisieren lässt.

Empirische Daten aus dem Unterricht bieten für eine sprachbewusste Lehramtsausbildung einen in mehrfacher Hinsicht interessanten Lerngegenstand: Sie lassen Einblicke in unterschiedliche Formen der fachlichen Kommunikation zu, ermöglichen eine Rekonstruktion von Lernprozessen und können wertvolle fach- und sprachdiagnostische Auskünfte geben. In $\triangleright$ Abschn. 2.4 wird daher der Fokus auf Lernerdaten als Analyseund Lerngegenstand gelegt.

- Abschn. 2.5 widmet sich einem aus hochschuldidaktischer Sicht zentralen Paradigma: dem forschenden Lernen. Am Beispiel des Praxissemesters wird aufgezeigt, wie eine Verzahnung der Aktivitäten an den Lernorten Schule und Universität aussehen kann und wie Studierende bei kleineren empirischen Studien im Bereich Sprachbildung im Fach Mathematik unterstützt werden können. Abschn. 2.6 knüpft daran an und vertieft das für viele Studierende besonders herausfordernde Thema der Entwicklung von Forschungsfragen. An studentischen Beispielen wird gezeigt, wie das Finden, Überprüfen und Präzisieren von Forschungsfragen an der Schnittstelle von Fach und Sprache unterstützt werden kann.

\subsection{Sprache im Fach erleben}

॥ Wir kommen ja durch das DaZ-Modul allgemein mit Sprachbildung in Berührung, aber eben nicht spezifisch in Verbindung mit dem Fach Mathematik. Ich finde, es ist schon wichtig, dass man den ganz klaren Bezug zu seinem Fach gewinnt (Studentin, Praxissemester 2019).

Fachliche Spiele bieten einen geeigneten Anlass, um die vorhandenen Kenntnisse und Erfahrungen der Studierenden in Bezug auf Sprache und sprachliches Handeln allgemein sowie speziell mit Blick auf das jeweilige Fach zu aktivieren. Ausgehend von der Untersuchung ihres eigenen Sprachgebrauchs in der fachlichen Spielsituation diskutieren die Studierenden über sprachliche Anforderungen und Erwartungen im Fachunterricht; dabei kann an relevante Theoriebildungen angeknüpft werden. Darüber hinaus wird ein erster Zugang zur Arbeit mit empirischen Daten ermöglicht.

Im vorliegenden Abschnitt wird eine Spielsituation vorgestellt, die sich als Einstieg in ein Seminar zu Sprachbildung im Mathematikunterricht eignet. Dabei wird Leitidee L1 
zu fachbezogenem Sprachwissen als Voraussetzung für sprachbildenden Fachunterricht ( Abschn. 1.2.1) konkretisiert. Aus hochschuldidaktischer Perspektive wird einerseits eine fachliche und zugleich praxisnahe Einstimmung in das Thema „Daten und Zufall“ ermöglicht. Andererseits werden verschiedene Facetten von Sprache verdeutlicht, die im Fachunterricht und insbesondere im mathematischen Sachgebiet Stochastik eine Rolle spielen. Die Studierenden gewinnen dabei erste Einsichten zur Fachspezifik von Sprache, z. B. hinsichtlich der Merkmale und Funktionen von Alltags- und Fachsprache. Der eigene - durch die Spielsituation weitgehend intuitive - Sprachgebrauch kann als Analysegegenstand genutzt werden; es wird also ,entdeckendes Lernen“ auf Hochschulebene ermöglicht (methodische Dimension). Darüber hinaus kann zur Reflexion der eigenen, ggf. mehrsprachigen Sprachlernerfahrungen und deren Bedeutung beim fachlichen Lernen angeregt werden (L1-2). Hinsichtlich der hochschuldidaktischen Design-Prinzipien wird in diesem Abschnitt die Integration von Sprache und Fach sowohl auf struktureller und planungsbezogener Ebene (DP1) als auch auf sozialer Ebene zur Gestaltung von Arbeitsprozessen (DP3) thematisiert.

\subsubsection{Sprache in Spielsituationen}

Das einleitende Zitat einer Studierenden aus einem Seminar zur Vorbereitung eines Schulpraktikums bringt ein häufig zu beobachtendes Dilemma der Hochschullehre auf den Punkt: Studierenden fällt es schwer, die Verknüpfung zwischen Studieninhalten und beruflich relevanten Situationen herzustellen. Nicht selten entsteht so der Eindruck, dass theoretische Konzepte erlernt werden, die mit der eigenen Professionalisierung nur wenig zu tun haben. Dies gilt mitunter auch für die Umsetzung von Konzepten und Methoden aus dem Bereich Sprachbildung im Fachunterricht. Umso wichtiger ist es, in fachbezogenen Veranstaltungen wie dem hier beschriebenen interdisziplinären Ansatz diese Verknüpfung explizit herauszuarbeiten.

Für einen praxisnahen und motivierenden Einstieg in das Thema „Sprachbildung im Fach" eignen sich fachliche Spiele aus dem Schulunterricht. Denn zum einen erleben die Studierenden den Zusammenhang von fachlichen Inhalten und sprachlichem Handeln selbst als Akteure in einer Spielsituation. Zum anderen reflektieren sie diesen Zusammenhang mit Blick auf reale Unterrichtssituationen. Die Studierenden werden also im Sinne der in Abschn. 1.2 formulierten Leitidee L1 für Sprache im Fach sensibilisiert. Im Folgenden wird ein solches Spielszenario für die Lehramtsausbildung Mathematik vorgestellt.

\section{- Zufallsspiel mit Sprachbeobachtung}

Das Spielszenario sieht vor, dass die Studierenden zunächst in Gruppen das Spiel „Die beste Wahl gewinnt“" aus dem Lehrwerk Mathewerkstatt 8 (Hußmann et al. 2015) spielen. Das Spiel ist für den Mathematikunterricht der Jahrgangsstufe 8 für die Leitidee „Daten und Zufall" konzipiert und stellt eine offene Erkundungssituation dar; die Schüler*innen sollen darin erste Erfahrungen mit theoretischen und empirischen Gewinnchancen sammeln, die Gewinnchancen unterschiedlicher Zufallsgeräte (Würfel, Reißzwecke usw.) vergleichen und Ansätze zur Quantifizierung von Gewinnchancen entwickeln (Hußmann 2015). 
- Abb. 2.1 Fachliche Spiele im Seminar

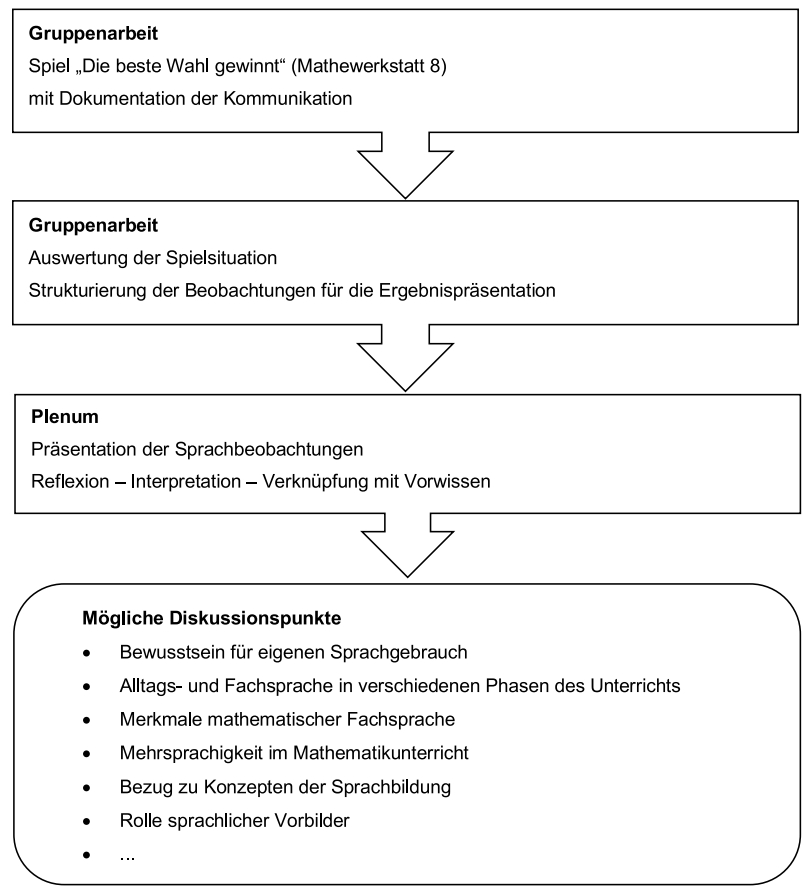

Für die Zwecke des Seminars wird für jede Gruppe ein Sprachbeobachter bzw. eine Sprachbeobachterin bestimmt, der/die den Auftrag erhält, die Kommunikation im Spiel genau zu beobachten und zu dokumentieren. Bei der anschließenden gemeinsamen Reflexion und Auswertung werden diese Beobachtungen von den Mitspieler*innen ergänzt, systematisiert und diskutiert.

- Abb. 2.1 veranschaulicht die Vorgehensweise im Seminar.

Die Studierenden erhalten zunächst den Auftrag, das Spiel in Gruppen à vier bis fünf Personen in mehreren Durchläufen zu spielen. Wie erwähnt, liegt die Besonderheit dieser hochschulischen Variante des Spiels darin, dass jeweils ein Gruppenmitglied das Spielgeschehen aus sprachlicher Sicht beobachtet. Er/sie erhält dafür den folgenden - bewusst offen gehaltenen - Beobachtungsauftrag:

\section{Arbeitsauftrag „Sprachbeobachtung im fachlichen Spiel“}

Sie haben den Auftrag, das Spielgeschehen zu beobachten. Hören Sie genau auf die Kommunikation zwischen den Spieler*innen und machen Sie Notizen. Welche sprachlichen Mittel nutzen die Spieler*innen? Welche Situationen sind sprachlich interessant und/oder herausfordernd?

Nach der Spielphase sprechen die Kleingruppen über ihren Sprachgebrauch während des Spiels, ergänzt um die Eindrücke der beobachtenden Person. Anschließend präsentiert jede Gruppe die Ergebnisse ihrer Sprachbeobachtung zusammenfassend im Plenum. 
Im Folgenden werden die Ergebnisse zweier Seminargruppen vorgestellt und Ansatzpunkte für die Reflexion und Diskussion aufgezeigt. In der Kommentierung der studentischen Produkte werden die folgenden Schwerpunkte gesetzt:

- Verhältnis von Alltags- und Fachsprache

- Fachwörter mit alltagssprachlicher Entsprechung

- Merkmale mathematischer Fachsprache

- Sprachliche Vorbilder

- Mehrsprachige Äußerungen im fachlichen Spiel

\subsubsection{Sprachbeobachtungen im fachlichen Spiel}

- Abb. 2.2 zeigt die Übersicht, die die Studierenden aus Seminargruppe 1 im Anschluss an das Spiel selbst erstellt haben.

Im Folgenden wird aufgezeigt, unter welchen Gesichtspunkten diese von den Studierenden genannten Aspekte im Seminargespräch aufgegriffen und mit Blick auf Unterrichtssituationen vertieft diskutiert werden können.

\subsubsection{Zum Verhältnis von Alltags- und Fachsprache}

Wie die Notizen in - Abb. 2.2 zeigen, haben die Studierenden der Seminargruppe 1 zwei Kategorien erstellt, um ihre Spielkommunikation systematisch darzustellen: „Mathematische Fachsprache“ und „Alltagssprache“. Die Funktion der Alltagssprache liegt dabei in der Interpretation der Studierenden in der „Unterstützung für das Verständnis“ - im Unterschied zur Fachsprache, deren Nutzen sie in der Beschreibung ,konkreter Sachverhalte“ sehen (- Abb. 2.2, Spalte „Beschreibung“). Alltagssprachliche Mittel stellen in der Tat eine hilfreiche und wichtige Grundlage für die Annäherung an und die Verständigung

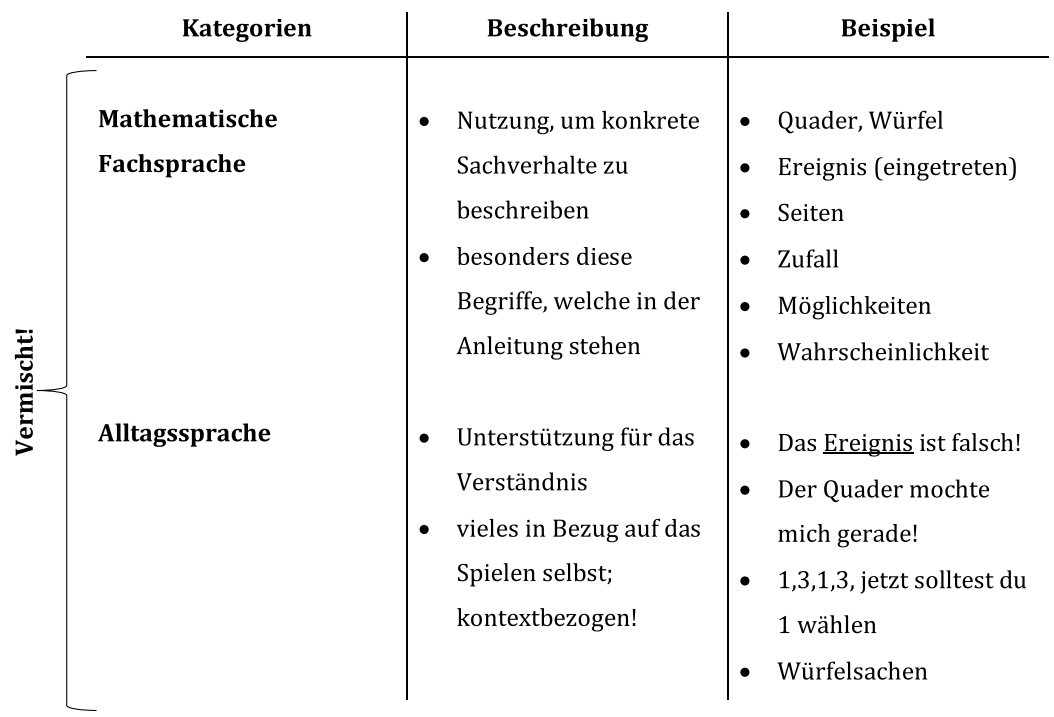

- Abb. 2.2 Sprachbeobachtung im Spiel (Seminargruppe 1) 
über fachliche Inhalte dar. So können sich Lernende in einer Erkundungsphase fachlichen Gegenständen zunächst alltagssprachlich nähern (im studentischen Beispiel selbst findet sich z. B. der Ausdruck „Würfelsachen“ für ,Zufallsgeräte“), bevor sie dann in der Systematisierungsphase schrittweise zu einer fachlichen Ausdrucksweise herangeführt werden (Auf- und Ausbau fachlicher Kommunikationsfähigkeit). Falls die Studierenden das Scaffolding-Modell bereits kennen, kann daran erinnert werden, dass alltagssprachliches, mündliches Kommunizieren auch dort als Ausgangspunkt für fachliches Lernen genannt wird (Gibbons 1998; dt. 2006; Kniffka und Neuer 2008; vgl. $>$ Abschn. 1.1.1).

Die Studierenden heben auch hervor, dass die Kategorien „Alltagssprache“ und „mathematische Fachsprache“ nicht voneinander zu trennen sind, sondern ,vermischt“ auftreten (die geschweifte Klammer links in - Abb. 2.2). Dies ist eine wichtige Beobachtung, die Anlass zur Diskussion geben kann, in welchem Verhältnis Alltags- und Fachsprache im Unterricht zueinander stehen und was entsprechend von den Lernenden erwartet werden kann und soll. Dabei lässt sich unterscheiden nach:

- mündlichen vs. schriftlichen Kommunikationssituationen (z. B. Diskurs während der Gruppenarbeit vs. schriftliche Dokumentation der Gruppenarbeit),

- unterschiedlichen Phasen des Unterrichts (z. B. Erkundungs-, Systematisierungs- oder Vertiefungsphase),

- Rezeption (z. B. beim Lesen eines Fachtexts) vs. Produktion (z. B. beim Schreiben im Lerntagebuch),

- unterschiedlichen Textsorten (z. B. „Brief“1 vs. mathematische Argumentation).

Es geht also um die zentrale Frage, welche Funktionen Alltags- und Fachsprache beim fachlichen Lernen haben, wie sie ineinandergreifen und wie sich die Anteile an Alltagsund Fachsprache im Zuge der fachlichen Ausdifferenzierung eines Lernbereichs verändern.

\subsubsection{Fachwörter mit alltagssprachlicher Entsprechung}

Einen zweiten Anknüpfungspunkt für die Diskussion im Rahmen der Lehrveranstaltung können die Fachausdrücke bilden, die die Studierenden als Beispiele in der Kategorie „Mathematische Fachsprache“ notiert haben: „Quader“, „Ereignis (eingetreten)“, „Seiten“, „Zufall“, „Möglichkeiten“, „Wahrscheinlichkeit“. ${ }^{2}$ Dabei fällt folgendes auf: Mit einer Ausnahme (nämlich „Quader“) haben alle der aufgeführten Fachausdrücke eine Entsprechung in der Alltagssprache. Der Unterschied zwischen der alltäglichen und der fachlichen Bedeutung eines Wortes ist manchmal relativ leicht zu erkennen. So ist ein mathematisches „Ereignis“ (z. B. „Die gewürfelte Zahl ist ungerade“) klar von einem Sport- oder Musikereignis zu unterscheiden. Manchmal ist die Abgrenzung von der alltäglichen Bedeutung weniger offensichtlich, etwa bei den Begriffen „Zufall“ und „Wahrscheinlichkeit"“ 3 Solche Beobachtungen lassen sich auch leicht auf andere Bereiche der Mathematik sowie auf andere Fächer ausweiten. Es bietet sich an, im Seminargespräch an einem Beispiel alltägliche und fachliche Bedeutungsausprägungen herauszuarbeiten. Dann zeigt sich schnell, dass etwa der Begriff ,,wahrscheinlich“ in vielen alltäglichen Zu-

1 Vgl. Abschn. 2.4.3.

2 „Würfel“ ist hingegen im gegebenen Kontext des Spiels (anders als im Kontext Geometrische Körper) gerade nicht als Fachwort zu kategorisieren.

3 Krüger et al. (2015, S. 217 ff.) zum Zufallsbegriff in Alltag, Fachwissenschaft und Unterricht. 
sammenhängen mit „,hoher Wahrscheinlichkeit“ gleichgesetzt wird („Morgen regnet es wahrscheinlich"), während der fachliche Wahrscheinlichkeitsbegriff an sich noch nichts über die Sicherheit des Eintretens eines Ereignisses aussagt (Krüger et al. 2015, S. 69).

In mehrsprachigen Seminargruppen können Vergleiche mit anderen Sprachen gezogen werden, um zu untersuchen, wie das Verhältnis von Alltags- und Fachwort dort jeweils organisiert ist. Erkan Gürsoy hat dies am Beispiel des Begriffs „Wahrscheinlichkeit“ im Türkischen ausgeführt:

\begin{abstract}
„Wahrscheinlichkeit" im Türkischen (erläutert von Erkan Gürsoy)
Im Türkischen existieren zwei synonyme Fachwörter für „Wahrscheinlichkeit“ - eines türkischen Ursprungs („olasılık“) und eines arabischen Ursprungs (,ihtimal“). Beide Ausdrücke sagen nichts über die Sicherheit des Eintretens eines Ereignisses aus; um eine hohe Wahrscheinlichkeit zum Ausdruck zu bringen, benötigt man wie im Deutschen ein Adjektiv (,,büyük“ = groß; ,büyük bir olasılıkla/ihtimalle“ = mit hoher Wahrscheinlichkeit). Beide Verwendungsweisen finden sich jedoch auch in der Alltagssprache, es handelt sich also auch im Türkischen nicht um Ausdrücke, die als fachsprachlich ins Auge springen. Darüber hinaus gibt es in der Alltagssprache die Ausdrücke „herhalde“ und „muhtemelen“ (arabischen Ursprungs), die beide ,,mit hoher Wahrscheinlichkeit" bedeuten.
\end{abstract}

\title{
2.1.2.3 Merkmale mathematischer Fachsprache
}

Die Notizen der Studierenden könnten auch Anlass zu einem Gespräch darüber sein, was eigentlich unter „,mathematischer Fachsprache“ genau zu verstehen ist. Denn es fällt auf, dass nahezu alle Ausdrücke, die die Studierenden in diese Kategorie aufgenommen haben, Fachtermini bzw. genauer: fachsprachliche Substantive sind (,Quader“, „Ereignis“ usw.). Dies erscheint insofern naheliegend, dass Fachtermini und insbesondere fachliche Substantive im Allgemeinen als typische Vertreter der Fachsprache gelten (Ehlich 1994), während andere fachsprachlich relevante Mittel leicht aus dem Blick geraten. ${ }^{4}$ Umso bemerkenswerter ist, dass den Studierenden das Verb „eintreten“ in Verbindung mit dem Fachbegriff „Ereignis“ aufgefallen ist (- Abb. 2.2, rechte Spalte). Die fachspezifische Kollokation ,ein Ereignis tritt ein" kommt, auch wenn die Bestandteile jeweils auch in der Alltagssprache zu finden sind, ausschließlich in der mathematischen Fachsprache im Kontext der Wahrscheinlichkeitstheorie vor. Die einzelnen Bestandteile können nicht variiert werden: Weder lässt sich „eintreten“ durch ein anderes Verb ersetzen (man kann also z. B. nicht sagen: *,ein Ereignis kommt vor" oder *,ein Ereignis tritt auf“) noch kann anstelle von „Ereignis“ ein anderes Substantiv stehen (man kann also z.B. nicht sagen: *,eine Menge tritt ein.“). Beobachtungen wie diese können zum Anlass genommen werden, um mit den Studierenden darüber zu sprechen, welche sprachlichen Mittel - jenseits von Fachtermini - im Unterricht berücksichtigt werden sollten, um Lernenden beim Auf- und Ausbau differenzierter sprachlicher Fähigkeiten im Fach Mathematik zu helfen (vgl. Leitidee L1-1).

4 Guckelsberger und Schacht (2018) für den Lernbereich Bedingte Wahrscheinlichkeiten sowie übergreifend für das Fach Mathematik Maier und Schweiger (1999) bzw. den Mathematikunterricht Prediger (2020). Vgl. auch Leitidee L1-1. 


\subsubsection{Sprachliche Vorbilder}

Einen weiteren Anknüpfungspunkt für die Diskussion im Rahmen der Lehrveranstaltung bietet die interessante Beobachtung (ebenfalls unter der Kategorie „mathematische Fachsprache“), dass die Studierenden im Spiel „,besonders diese [Fach-]Begriffe“ verwendeten, „welche in der [Spiel-]Anleitung stehen“ (- Abb. 2.2). Dies gibt Anlass zu Überlegungen, inwiefern sprachliche Vorbilder - etwa Formulierungen in Lehrwerken, sprachliche Modelle und Hilfen sowie das sprachliche Vorbild der Lehrkraft - die schülerseitige Kommunikation beeinflussen und was das für das eigene sprachliche Handeln als Lehrkraft bedeutet (Leitidee L3).

Dass sprachliche Vorbilder die Kommunikation von Lernenden deutlich beeinflussen können (und zwar auch in unerwarteten sprachlichen Bereichen, wie z. B. beim Gebrauch von Konnektoren), zeigen Befunde aus experimentellen Erhebungen zum sprachlichen Handeln in der Primarstufe (Redder et al. 2013). Die Autorinnen sprechen sich dafür aus, sprachlich reichhaltige Lernumgebungen zu schaffen. Hingegen erweise sich ,[e]ine Vereinfachung der Unterrichtskommunikation zu didaktischen Zwecken, insbesondere zur Unterstützung sprachlich schwächerer oder unsicherer Schüler, [...] als nicht zielführend." (Redder et al. 2013, S. 119)

Nicht zuletzt kann in diesem Zusammenhang auch der Bogen zu sprachbildenden Ansätzen wie der Genredidaktik geschlagen werden, die den Umgang mit Fachtexten über sprachliche Modellierung fördern (Gibbons 2015; Gürsoy 2018; Jahn 2020).

\subsubsection{Mehrsprachige Äußerungen im fachlichen Spiel}

Das zweite Beispiel nimmt die Beobachtungen einer studentischen Seminargruppe in den Blick, die sich durch die Zweisprachigkeit aller Gruppenmitglieder auszeichnet.

Die in - Abb. 2.3 wiedergegebenen Notizen ${ }^{5}$ sind in einer studentischen Spielgruppe entstanden, deren Mitglieder deutsch-türkisch bilingual sind und während des Spiels beide Sprachen nutzten.

In ihren Notizen zitieren sie zwei Äußerungen mit einem Sprachwechsel zwischen Deutsch und Türkisch:

- Ich hab's geschafft bak [schau!]

- Demnächst werde ich das auch probieren ama [aber] warte, başka ne var? [was gibt es noch?]

Die Studierenden selbst vermuten, dass sie im Spiel „,bei Verständnisproblemen und Uneinigkeiten“ zwischen den Sprachen wechselten, sowie insbesondere auch - so erläuterten sie im Seminargespräch - ,wenn es emotional wurde“. Diese Beobachtung ist interessant, weil sie darauf hinweist, dass nicht beliebig zwischen den Sprachen gewechselt wird, Sprachwechsel also funktional sind (Keim 2008, 2012). So könnte man die Äußerung „Ich hab's geschafft bak [, schau!']“ dahingehend interpretieren, dass ins Türkische gewechselt wird, um die Aufmerksamkeit der Mitspieler*innen für den Erfolg im Spiel zu erlangen. Vergleichbare Beobachtungen machen Grießhaber et al. (1996) zur Kommunikation unter Türkisch und Deutsch sprechenden Schüler*innen im Unterricht: „Auch Ausrufe der Überraschung, der Erkenntnis, des Erstaunens, der Verwunderung

5 Es werden hier nur diejenigen Auszüge wiedergegeben, die sich auf die mehrsprachige Kommunikation der Studierenden beziehen. Die Ergebnisse der Sprachbeobachtung betrafen ansonsten ähnliche Aspekte wie die in Beispiel 1 ( Abschn. 2.1.2) genannten. 
- Abb. 2.3 Sprachbeobachtung im Spiel (Seminargruppe 2; Auszug)
- Kommunikation teilweise auf türkisch

- Muttersprache - Zweitsprache

- Bei Verständnisproblemen, Uneinigkeiten => switching

Beispiele:

- „Ich hab's geschafft bak“

- „Demnächst werde ich das auch probieren ama warte, başka ne var?“

usw. (...), mit denen die Schüler die Mitschüler auf die emotionale Verarbeitung bestimmter Probleme lenken - charakteristisch für kooperative Gruppenarbeit ohne Lehrer - sind fast durchgehend türkisch." (Grießhaber et al. 1996, S. 11) Es zeigen sich also interessante Parallelen zwischen dem studentischen und dem schulischen Diskurs.

Mehrsprachige Äußerungen wie die oben genannten führen in der Hochschullehre in der Regel zu einer Diskussion über die Verwendung von zwei oder mehr Sprachen innerhalb einer Äußerung oder Kommunikationssituation. Dabei sollte deutlich werden, dass das Wechseln zwischen Sprachen eine normale Handlungspraktik Mehrsprachiger darstellt und Zeichen für eine hohe Sprachkompetenz ist: „, $[.$. ] codeswitching requires a high level of cognitive control, involving neural networks known as the executive system in the brain, as well as a good knowledge of the grammatical systems of the different languages involved." (Wei 2013, S. 366)

Die Beobachtungen der Studierenden sind aber auch unter einem anderen Gesichtspunkt relevant: Manche Lehrkräfte lassen neben der Unterrichtssprache Deutsch nur ungerne andere Sprachen zu, weil sie befürchten, dass die Lernenden sich über fachfremde Inhalte austauschen (Heinemann und Dirim 2016). Das Beispiel aus der Seminargruppe legt das Gegenteil nahe: Gerade die Nutzung des Türkischen spiegelt die hohe Involviertheit der Studierenden in die Aufgabe.

An dieser Stelle kann folglich in der Hochschullehre der Bogen zu didaktischen Potentialen der Einbeziehung mehrsprachiger Ressourcen im Mathematikunterricht geschlagen werden (Redder et al. 2018; Duarte 2019; Prediger et al. 2019b).

\section{Impulse für die Anschlussdiskussion}

Durch die Spielsituation und den damit verbundenen Wettbewerb kommunizieren die Studierenden spontan und es ist i.d.R. kein Bemühen um eine fachlich erwünschte Ausdrucksweise erkennbar. Die Äußerungen sind dadurch in vielerlei Hinsicht mit denen von Schüler*innen im Unterricht vergleichbar. So lassen sich die Ergebnisse im Weiteren dann auch mit Blick auf die Realsituation Unterricht reflektieren:

- Welchen Sprach(en)gebrauch können wir von Schüler*innen in einer Spiel- bzw. Erkundungsphase erwarten bzw. nicht erwarten?

- Wie lassen sich alltagssprachliche Äußerungen aufgreifen und fachsprachlich (z. B. in einer Systematisierungsphase) weiterführen?

- Welche Vermutungen lassen sich aus der Beobachtung von Schüleräußerungen aufstellen (z. B. hinsichtlich zugrundeliegender Vorstellungen)?

- Wie kann die Mehrsprachigkeit der Schüler*innen im Unterricht als Ressource genutzt werden? 


\subsection{Aufgaben sprachbewusst planen}

॥ Das meine ich mit „Leichtigkeit“ in Bezug auf das Thema Sprachbildung: Dass es gar nicht so schwer ist, dass man keinen Riesenberg vor sich sehen muss als Student oder als Lehrer. Dass man eben schon mit Kleinigkeiten etwas bewirken kann (Studierende, Praxissemester 2019).

In der Lehramtsausbildung Mathematik spielen die Analyse, Konstruktion, Variation und Erprobung von Aufgaben eine wichtige Rolle. In diesem Zusammenhang lohnt eine Betrachtung der Rolle von Sprache sowie der Verknüpfung mathematischer und sprachlicher Lerngelegenheiten, denn der Umgang mit Aufgaben(texten) im Mathematikunterricht stellt viele Schüler*innen vor große Herausforderungen. Dieser Abschnitt gibt Anregungen, wie die Arbeit mit und an schulischen Aufgabenstellungen, die das Zusammenspiel von Sprache und Fach gezielt adressieren, in der Hochschullehre thematisiert werden kann. Insofern wird die Leitidee L2 zum sprachbewussten Umgang mit Aufgaben konkretisiert. Adressiert wird dabei etwa die Rolle von typischen Aufgabenformaten vor dem Hintergrund unterschiedlicher Kernprozesse sowie deren fachliche und sprachliche Anforderungen an Lernende, sodass die Studierenden das Potential von Aufgaben unter Berücksichtigung mathematikdidaktischer und sprachbezogener Konzepte reflektieren (L2-1). Auf dieser Grundlage werden dann Variationsmöglichkeiten mit Blick auf sprachbildende Zugänge diskutiert (L2-2), die Studierende im Kontext entsprechender Lehrveranstaltungen vornehmen können (L2-3).

Hinsichtlich der hochschuldidaktischen Design-Prinzipien wird in diesem Abschnitt die Integration von Sprache und Fach vor allem auf inhaltlicher Ebene zur Arbeit mit authentischen Lerngegenständen thematisiert (DP2).

\subsubsection{Aufgabenvariationen mit mehr "Leichtigkeit"}

Das einleitende Zitat einer Studierenden aus einem Begleitseminar zum Praxissemester beschreibt eine häufig zu beobachtende Wahrnehmung des Themas Sprachbildung aus Sicht der Schulpraxis: Es wird als „Riesenberg“ gesehen, als ein Thema also, das so fundamentale Konsequenzen für das eigene unterrichtliche Handeln hat, dass viele Studierende und Lehrkräfte einfach nicht wissen, wo sie genau anfangen sollen. Die Studierende berichtet hingegen von Erfahrungen aus dem Praxissemester, die dem Thema mehr „Leichtigkeit“ verleihen, mit der Erkenntnis: Auch kleine Veränderungen im Unterricht können schon viel bewirken. Das vorliegende Kapitel gibt einige Anregungen, wie Aufgabenvariationen in interdisziplinären Lehrveranstaltungen erprobt werden können.

Aufgaben spielen eine zentrale Rolle in der Schule - dies gilt insbesondere auch für den Mathematikunterricht. Entsprechend ist die Auseinandersetzung mit Aufgaben und ihren Potentialen, Merkmalen und Funktionen ein wichtiger Bestandteil des Lehramtsstudiums Mathematik - und zwar für jede Schulform und -stufe. Dass auch Studierende das Thema als relevant für ihre Professionalisierung einschätzen, zeigt die Rückmeldung einer Mathematikstudentin im Praxissemester 2019: 
॥ Ich fand das Thema Aufgabengestaltung und Aufgabenvariation super spannend. Mir sind in Mathebüchern schon oft Aufgaben aufgefallen, die nicht gut formuliert und für die Schüler unmotivierend waren. Jetzt habe ich einen kleinen Methodenpool, wie ich Aufgaben mit einfachen Mitteln selbst umgestalten oder umstrukturieren kann (Studierende, Praxissemester 2019).

Mathematikaufgaben unterscheiden sich je nachdem, in welchen Prozessen - etwa beim Problemlösen, Modellieren, Argumentieren oder Begriffsbilden (Büchter und Leuders 2016) - und in welchen Funktionen - z. B. zur Diagnose, zum Lernen oder im Rahmen von Leistungssituationen - sie verwendet werden. In Verbindung mit dem Anspruch an einen sprachbewussten Mathematikunterricht bekommt die Auseinandersetzung mit Aufgaben noch einmal besonderes Gewicht. Eine große Herausforderung stellen in diesem Zusammenhang Textaufgaben dar: Vielfältige Studien weisen auf die Hürden hin, die sich für Schülerinnen und Schüler ergeben, und zwar sowohl in sprachrezeptiven als auch in sprachproduktiven Situationen (z. B. Wilhelm 2016; Prediger et al. 2015; Reusser 1997). Textaufgaben werden nicht nur schlechter gelöst als vergleichbare Aufgaben z. B. in numerischer Darstellungsform (Reusser 1997). Vielmehr erweist sich die Sprachkompetenz als zentrale Variable für die Erklärung von herkunftsbedingten Leistungsdisparitäten, insofern sie einen deutlich stärkeren Zusammenhang zur Mathematikleistung aufweist als etwa andere soziale Faktoren wie Migrationshintergrund oder Zeitpunkt des Deutscherwerbs (Prediger et al. 2015).

Nicht selten ist eine Reaktion auf solche Befunde, dass man gerade in der Mathematik als einem vermeintlich ,spracharmen“ Fach doch weitgehend auf Sprache verzichten könne. Der Ansatz von Aufgabenkaskaden spiegelt ein solches Verständnis (• Abb. 2.4).

Ein solcher Ansatz, der Sprache bewusst zu umgehen versucht, ist aber aus mehreren Gründen kontraproduktiv. Denn eine Reduktion auf kalkül- und eher verfahrensbezogene Zugänge setzt häufig weniger auf das Verständnis der mathematischen Zusammenhänge als vielmehr darauf, bestimmte Verfahren zu trainieren; das kann allerdings z. B. fehlerhafte Routinen sogar noch verstärken. Umgekehrt wird der Gegenstandsbereich mitnichten vereinfacht, wenn Sprache reduziert wird - im Gegenteil: Für verstehens- und vorstellungsorientierte Zugänge zur Mathematik braucht es geeignete Sprachmittel, um die Inhalte zu verstehen. Insofern ergibt sich gerade für das Fach Mathematik die Forderung nach der Vermittlung von Sprachkompetenz (z. B. Prediger 2009). Vor diesem Hintergrund ist es sehr zu begrüßen, dass Sprachbildung auch aus curricularer Perspektive als „Querschnittsaufgabe aller an schulischer Bildung Beteiligten und durchgängiges Unterrichtsprinzip in allen Fächern, Lernbereichen und Lernfeldern“ (KMK 2019, S. 4) anerkannt und ausgewiesen ist. Für eine Darstellung der spezifischen Sprachmittel, die

\section{Berechne}

\begin{tabular}{|c|c|c|c|c|c|c|}
\hline $\begin{array}{r}10 \cdot 10 \\
7 \cdot 10 \\
5 \cdot 10\end{array}$ & $\begin{array}{l}9 \cdot 8 \\
5 \cdot 8 \\
3 \cdot 8\end{array}$ & $\begin{array}{l}10 \cdot 7 \\
6 \cdot 7 \\
5 \cdot 7\end{array}$ & $\begin{array}{l}9 \cdot 6 \\
8 \cdot 6 \\
4 \cdot 6\end{array}$ & $\begin{array}{l}10 \cdot 5 \\
9 \cdot 5 \\
8 \cdot 5\end{array}$ & $\begin{array}{l}10 \cdot 4 \\
6 \cdot 4 \\
3 \cdot 4\end{array}$ & $\begin{array}{l}\cdot 3 \\
5 \cdot 3 \\
3 \cdot 3\end{array}$ \\
\hline $7 \cdot{ }_{-}=49$ & $8 \cdot{ }^{\prime}=72$ & $3 \cdot-=15$ & $2 \cdot{ }_{-}=20$ & $10 \cdot{ }_{-}=40$ & $6 \cdot{ }_{-}=48$ & $8 \cdot{ }_{-}=64$ \\
\hline $5 \cdot{ }_{-}=45$ & $6 \cdot-=36$ & $7 \cdot=42$ & $4 \cdot-=24$ & $3 \cdot-=21$ & $5 \cdot{ }_{-}=35$ & $2 \cdot{ }_{-}=24$ \\
\hline $3 \cdot{ }_{-}=27$ & $5 \cdot{ }_{-}=25$ & $7 \cdot{ }_{-}=56$ & $9 \cdot{ }_{-}=81$ & $8 \cdot{ }_{-}=32$ & $7 \cdot{ }_{-}=35$ & $3 \cdot{ }_{-}=12$ \\
\hline
\end{tabular}

- Abb. 2.4 Aufgabenkaskaden zur Multiplikation im Hunderterraum 
man im Mathematikunterricht jeweils hinsichtlich der unterschiedlichen mathematischen Inhaltsbereiche benötigt, sei etwa auf Prediger (2020) verwiesen.

Im Folgenden werden - pointiert - drei Strategien gegenübergestellt, die in der unterrichtlichen Praxis oder im Rahmen von Fortbildungen sehr häufig anzutreffen sind, wenn über die Rolle von Sprache im Fach diskutiert wird.

i. Sprache-vermeintlich-vermeiden: Unser Schulbuch eignet sich nicht für einen sprachbewussten Mathematikunterricht, die Aufgaben sind zu textlastig. Für Schülerinnen und Schüler mit sprachlichen Schwierigkeiten lasse ich das Schulbuch weg und gebe ihnen nur die Rechenaufgaben.

ii. Augen-zu-und-durch: Wir haben in der Fachgruppe die klare Verabredung, dass wir das Buch nutzen. Wenn die Aufgaben zu textlastig sind, müssen die Lernenden in entsprechenden Förderstunden die Lesekompetenzen verbessern. Neue Aufgaben stellen: Wie soll ich das leisten?

iii. Aufgabenvariation: Ich kenne Möglichkeiten zur Veränderung von Aufgaben. Mit einfachen Mitteln kann man manchmal schon sehr viel erreichen.

Wissenschaftliche Forschungsbefunde belegen klar, dass die Szenarien i und ii nicht zielführend sind. Die vermeintliche Vermeidung von Sprache (Szenario i) berücksichtigt nicht, dass es Sprache braucht, um sich die mathematischen Konzepte zu erarbeiten eine reine Reduktion auf (Rechen-)Verfahren wird der Komplexität der mathematischen Gegenstände nicht gerecht und enthält den Schülerinnen und Schülern letztlich auch fundierte Verstehensprozesse vor (Maier und Schweiger 1999; Zindel 2019). Szenario ii ist ebenso wenig zielführend. Sprache ist nämlich nicht nur Lernmedium und Lernvoraussetzung, sondern insbesondere auch ein Lerngegenstand im Mathematikunterricht (etwa Meyer und Prediger 2012; Pimm 1987; Ellerton und Clarkson 1996; Maier und Schweiger 1999). Dass fachliche Kommunikationsfähigkeit eine Kompetenz ist, die Lernende im jeweiligen Fachunterricht erlangen sollen, lässt sich den entsprechenden Curricula entnehmen. Eine ausschließliche Auslagerung etwa in den Deutschunterricht oder in eine allgemeine Sprachförderung ist schon allein deshalb nicht hilfreich, weil sprachliche Fähigkeiten nicht ohne Weiteres aus einem Fach in ein anderes übertragen werden können. Das zeigen beispielsweise Untersuchungen aus dem Projekt SchriFT (Schreiben im Fachunterricht unter Einbeziehung des Türkischen) zum Schreiben von Versuchsprotokollen im Physikunterricht der Jgst. 8:

॥ Sprachliche Fähigkeiten aus dem Deutschunterricht helfen Schülerinnen und Schülern [... ] nur im geringen Maße beim kohärenten Schreiben eines Versuchsprotokolls. Sprachliche Strukturen sollten demnach funktional in Verbindung mit dem Fachwissen und dem prozeduralen naturwissenschaftlichen Wissen im Physikunterricht vermittelt werden (Boubakri et al. 2018, S. 260).

Der folgende Abschnitt gibt Anregungen, wie eine sprachbewusste Aufgabenplanung in der Hochschullehre adressiert werden kann. Als handlungsleitende theoretische Konzepte werden dabei die Möglichkeiten der Darstellungsvernetzung genutzt sowie eine eher phasengebundene Betrachtung unterrichtlicher Prozesse entlang der Kernprozesse des Erkundens, des Systematisierens und des Übens.

Zur Produktion und zur Variation von Aufgaben gibt es vielfältige Anregungen (für ausführliche Darstellungen etwa Schupp 2002; Büchter und Leuders 2016). Für eine vertiefte Auseinandersetzung mit der Rolle von Sprache in der unterrichtlichen Praxis sind vielfältige Anregungen auch bei Prediger (2020) und Abshagen (2015) zu finden. 


\subsubsection{Sprachbewusste Aufgabenplanung in der Hochschullehre am Beispiel Mittelwerte}

Im Folgenden wird am Beispiel Mittelwerte exemplarisch gezeigt, wie sprachbewusste Aufgabenplanung und -variation in der Hochschullehre thematisiert werden kann - mit dem Ziel der Ausarbeitung von Konzepten für einen sprachbildenden Mathematikunterricht.

Die stoffdidaktische und sprachliche Einordnung des Themas wird zunächst anhand der Aufgabe „Mittelwert ist nicht gleich Mittelwert“ aus dem Lehrwerk Lambacher Schweizer vorgenommen ( Abb. 2.5; Abschn. 2.2.2.1). Anschließend werden von Studierenden entwickelte Aufgaben zum Thema Mittelwerte analysiert und diskutiert ( Abschn. 2.2.2.2).

Für einen thematischen Einstieg im Seminar können den Studierenden folgende Arbeitsaufträge gegeben werden:

\section{Arbeitsauftrag für die Hochschullehre zur Analyse von Aufgaben}

- Lösen Sie die Aufgabe. Welche fachlichen Anforderungen sind mit der Aufgabe verbunden?

- Übertragen Sie zwei Sätze aus der Aufgabe in eine Fremd- oder Herkunftssprache. Reflektieren Sie sprachliche Schwierigkeitsbereiche und Lösungsansätze.

- Welchen Stellenwert hat Sprache bei der Bearbeitung der Aufgabe (z. B. als Lernvoraussetzung, Lernmedium und Lerngegenstand)?

\subsubsection{Merkmale des stochastischen Kontexts „Mittelwerte“}

Bevor nun genauer die Frage diskutiert wird, wie die Arbeit an Aufgaben in Lehrveranstaltungen produktiv gestaltet werden kann, sei in Kürze zunächst auf den mathematischen Gehalt der Aufgabe „Mittelwert ist nicht gleich Mittelwert“ (• Abb. 2.5) eingegangen. Inhaltlich wird hier der Begriff des arithmetischen Mittels thematisiert. Der Begriff bezeichnet eine der wichtigsten statistischen Kenngrößen im Umgang mit Daten in der Schule. Von besonderer Bedeutung ist in diesem Zusammenhang vor allem die Erfahrung, die von der Frage ausgeht, wo sich in einem gegebenen Datensatz die Mitte befindet und wie diese bestimmt werden kann - hier unterscheidet man in der Mathematik unterschiedliche Mittelwerte, etwa den Median/Zentralwert, das harmonische Mittel, das geometrische Mittel oder eben das arithmetische Mittel. Letzteres wird gebildet als Quotient der Summe aller Werte geteilt durch die Anzahl der Werte. Für eine sehr lesenswerte Übersicht zu den inhaltlichen Vorstellungen zum arithmetischen Mittel sei auf Sill (2016) verwiesen. Der Aufbau tragfähiger Vorstellungen ist in diesem Zusammenhang besonders wichtig. Wird nämlich allein die Rechenvorschrift auswendig gelernt, so fehlt eine Vorstellung davon, warum dieser Begriff besonders nützlich ist, wozu er verwendet wird und was er genau - z. B. auch in Abgrenzung zu anderen Mittelwerten - bedeutet. Vor allem aber kann eine zu starke Betonung des Kalküls problematische Übergeneralisierungen fördern, d.h. die Lernenden wenden die auswendig gelernten Verfahren in falscher oder nicht zulässiger Weise an. Genau dies soll im Rahmen der obigen Aufgabe reflektiert werden. Die drei im Aufgabentext genannten Schülerinnen Johanna, Claudia und Lina ha- 
- Abb. 2.5 Aufgabe zum Thema Mittelwerte (Abschrift aus: Lambacher Schweizer 2017, S. 180)
Unterrichtsebene / Schulbuchaufgabe: „Mittelwert ist nicht gleich Mittelwert“

Drei Schülerinnen teilen sich die Arbeit bei der Meinungsumfrage: Johanna hat bei 10 Befragten 6 regelmäßige Leser $(60 \%)$, Claudia unter 30 Befragten 15 regelmäßige Leser $(50 \%)$ und Lina unter 20 Befragten 2 regelmäßige Leser gefunden (10\%). Johanna meint:

„Also lesen im Mittel $\frac{(60 \%+50 \%+10 \%)}{3}=40 \%$ regelmäßig die PopNews“. Larissa meint, das Ergebnis sei falsch. Sie kommt auf einen Mittelwert von $38,3 \%$. Nimm Stellung.

ben jeweils unterschiedlich viele Personen nach ihrem Leseverhalten befragt und jeweils für die betrachtete Teilmenge das arithmetische Mittel gebildet. Nun sollen die Ergebnisse zusammengeführt werden. Der Ansatz, dass die arithmetischen Mittelwerte addiert und dann durch die Anzahl (also 3) dividiert werden, ist nicht zulässig. Grund dafür ist, dass die Stichproben der drei Schülerinnen unterschiedlich groß sind. Für die Berechnung des arithmetischen Mittels wäre es notwendig, die Summe aller Lesenden durch die Anzahl aller befragten Personen zu dividieren, d. h. $\bar{x}=\frac{6+15+2}{10+30+20}=\frac{23}{60}=0,383=38,3 \%$. Insofern ist Larissas Behauptung aus der Aufgabe, dass der zunächst gewählte Ansatz falsch sei, richtig.

Betrachtet man die sprachliche Gestaltung der Aufgabe, so fällt als eine zentrale Herausforderung die Versprachlichung des Anteilbegriffs auf. So finden sich in dem Aufgabentext die folgenden Formulierungen:

- bei 10 Befragten 6 regelmäßige Leser

- unter 30 Befragten 15 regelmäßige Leser

Üblich sind darüber hinaus weitere Formulierungen, etwa „,bei 6 von 10 Befragten“, „,bei 6 der 10 Befragten“, „die Hälfte der Befragten“. Die Beispiele zeigen: Inhaltlich geht es in allen sprachlichen Varianten um den Anteil der Leserinnen und Leser an den befragten Personen. Allerdings zeigen sich unterschiedliche Akzentuierungen, die an Lernende nicht selten hohe Anforderungen stellen. So kann etwa die Leserichtung variieren (,6 von 10“, „,bei 10 Befragten 6 Leser“) oder der Gebrauch und die Rolle von Präpositionen („,bei x von y“; „,bei y Befragten x Leser“; „unter y Befragten x Leser"). Prediger (2020) spricht von bedeutungsbezogenen Begriffen (in diesem Fall die entsprechenden Präpositionen), die für das Verständnis der spezifischen mathematischen Begriffe (in diesem Fall des Anteil- bzw. Bruchzahlbegriffs) zentral sind.

Für Schülerinnen und Schüler ist es besonders wichtig, solche sprachlich verdichteten Ausdrücke wie „bei x von y“ oder ,unter y Befragten x Leser“ weiter aufzufalten (dazu etwa Zindel 2019; Prediger und Zindel 2017), z. B. durch die Visualisierung von Abhängigkeiten, durch die Generierung von Beispielen, mit Hilfe expliziter Argumentationsstrukturen oder durch Nachfragen, die gezielt die thematisierten Größen adressieren.

\subsubsection{Studentische Aufgabenvariationen}

Das o. g. Aufgabe „Mittelwert ist nicht gleich Mittelwert“ (• Abb. 2.5) kann in der Hochschullehre nicht nur als Analyse- und Reflexionsanlass genutzt werden, sondern in einem weiteren Schritt auch, um Aufgabenvariationen bzw. eigene Aufgabenplanungen zum Thema Mittelwerte zu initiieren. Der Arbeitsauftrag an die Studierenden lautet: 
Entwickeln Sie zum Thema „Mittelwerte“ - ggf. auf Grundlage der Aufgabe „Mittelwert ist nicht gleich Mittelwert" - jeweils eine Aufgabe für die Erarbeitungsphase, für die Konsolidierungsphase und für eine vertiefende Übephase. Beziehen Sie das Prinzip des Darstellungswechsels ein. Nutzen Sie dafür auch die Checkliste zur Sprachbildung.

Die Checkliste Sprachbildung (• Abb. 2.6) gibt den Studierenden praktische Anregungen für eine Überprüfung von bestehenden oder selbst entwickelten Aufgaben hinsichtlich sprachbezogener Aspekte; darüber hinaus kann sie natürlich auch als Anregung bei der Planung neuer Aufgaben(teile) dienen.

Das erste studentische Beispiel (- Abb. 2.7) stellt ein Gerüst für die Planung unterschiedlicher unterrichtlicher Handlungen zum Thema Mittelwerte dar. Der Student möchte im Rahmen einer Unterrichtssequenz die Mittelwerte ,arithmetisches Mittel“, „Median“ und „Modalwert“ thematisieren. Die Erkundungsphase soll im Kontext von Körpergrößen erfolgen. Dabei sollen die Schülerinnen und Schüler in Kleingruppen im Rahmen eines

- Abb. 2.6 Checkliste Sprachbildung
Checkliste Sprachbildung für die Konstruktion und Variation von Mathematikaufgaben

Liebe Studierende,

Sie können die folgenden Leitfragen nutzen, um bestehende oder von Ihnen neu entwickelte Aufgaben zu den unterrichtlichen Kernprozessen des Erkundens (Winter, 1989a; Gallin \& Ruf, 1998), Sicherns bzw. Systematisierens (z.B. Prediger et al., 2011a) und Übens (z.B. Leuders, 2011) auf Aspekte der Sprachbildung hin zu überprüfen und ggf. zu überarbeiten.

Die Fragen sollen Ihnen helfen, die Mathematikaufgaben aus sprachlicher Sicht einzuschätzen - Sie müssen die Fragen aber nicht „abarbeiten“. Natürlich dürfen Sie gerne weitere Aspekte mit einbringen.

Welche sprachlichen Besonderheiten hat die Aufgabe?

- Welche Begriffe sind zentral für das Verständnis?

- Kommen fachspezifische Substantiv-Verb-Verbindungen vor, die der besonderen Klärung bedürfen?

- Sind ungewohnte syntaktische Konstruktionen enthalten, die die Lernenden entschlüsseln müssen?

Welche Möglichkeiten aktiven sprachlichen Handelns bietet die Aufgabe?

- Bietet die Aufgabe Anlässe zur gemeinsamen Problemlösung?

- Gibt es authentische Sprechanlässe?

- Schreiben die Lernenden etwas (evtl. auch gemeinsam/kooperativ)? 
- Abb. 2.6 (Fortsetzung)
Inwiefern verlangt oder fördert die Aufgabe situationsentbundenes Sprechen oder Schreiben?

- Sind die Lernenden aufgefordert, im Plenum zusammenfassend über Ergebnisse einer Gruppenarbeit zu berichten?

- Wird zum Wechsel zwischen mündlichem und schriftlichem Sprachgebrauch aufgefordert?

Was ist die sprachliche Erwartung, die Sie mit der Aufgabe verbinden?

- Sollen die Lernenden bestimmte Fachbegriffe verwenden?

- Steht die Alltagssprache im Vordergrund?

- Wird eine bestimmte Textsorte gefordert?

Lassen sich mit der Aufgabe Schreibanlässe verbinden?

- Sollen die Lernenden etwas beschriften, eine Aufgabe selbst formulieren, aus einer Kalkülaufgabe eine Textaufgabe oder einen Zeitungsbericht entwickeln oder eine Definition erarbeiten?

Was dient der Sprachentlastung?

- Wird an Vorwissen der Lernenden angeknüpft?

- Wurde eine bestimmte methodische Aufbereitung / Einbettung zur Sprachentlastung gewählt?

- Werden sprachliche Beispiele / Vorbilder gegeben?

Wie werden die Lernenden aktiv in den Lernprozess eingebunden?

- Finden sie selbst Fragestellungen?

- Stellen sie Vermutungen auf?

- Sind sie an der Generierung / Sammlung von Daten beteiligt?

- Spielen digitale Werkzeuge eine Rolle?

Bietet die Aufgabe Gelegenheit zur Darstellungsvernetzung?

- Werden unterschiedliche Darstellungen genutzt?

- Werden Darstellungen miteinander vernetzt?

- Haben Lernende Gelegenheit, Darstellungen hinsichtlich ihrer Potentiale und Grenzen zu beurteilen?

Welche Möglichkeiten zur Kooperation und Reflexion bietet die Aufgabe?

- Vergleichen die Lernenden unterschiedliche Lösungsansätze?

- Überarbeiten / kommentieren die Lernenden die Vorgehensweisen / Ergebnisse von anderen?

- Vergleichen die Lernenden die Schwierigkeit von Aufgaben? 


\section{Mittelwerte}

Entdecken, Erfinden, Erkunden:

Was ist die mittlere Körpergröße innerhalb der Gruppen?

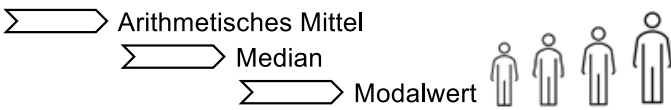

Sichern und Systematisieren:

Wie unterscheiden sich die Mittelwerte?

$\sum$ Durchschnitt

$\sum$ Wert in der Mitte

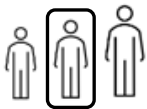

$\sum$ Häufigster Wert

Üben:

Wie ändern sich die Mittelwerte, wenn eine Playmobilfigur als Schüler hinzukommt? geringe Änderung / starke Änderung / keine Änderung

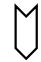

Verhalten bei Ausreißern

- Abb. 2.7 Studentisches Unterrichtsgerüst „Mittelwerte“

entdeckenden Lernprozesses der Leitfrage nachgehen: „Was ist die mittlere Körpergröße innerhalb der Gruppen?" Im Rahmen der anschließenden Sicherungsphase soll dann herausgearbeitet werden, wie sich die jeweiligen Mittelwerte unterscheiden. Dafür ist es entscheidend, auf die inhaltliche Unterscheidung von Durchschnitt, Wert in der Mitte und häufigstem Wert bewusst zu achten. Für die anschließende Übephase ist eine Aktivität geplant, bei der die Lernenden eine Playmobilfigur als fiktiven Schüler hinzunehmen und ermitteln, wie sich die Werte ändern. Hier wird also darauf eingegangen, dass die Mittelwerte jeweils unterschiedlich stark auf Ausreißer - also auf extreme Werte - reagieren. So ändert sich das arithmetische Mittel bei Ausreißern stark, während der Median in seiner Eigenschaft als Zentralwert z. B. auch gleich bleiben kann (aber nicht muss).

Bei der studentischen Skizze in - Abb. 2.7 handelt es sich zunächst um ein Gerüst, das noch keine konkreten Aufgabenstellungen bereithält. Es wurde im Rahmen der Lehrveranstaltung genutzt, um Aufgaben auszuarbeiten, die auch die unterschiedlichen Kernprozesse berücksichtigen. So entwickelte eine Studentin eine Aufgabenvariation für die Sicherungsphase und erprobte sie im Praxissemester in einer 7. Klasse. Dabei wurden die Schüler*innen im Anschluss an eine Lerneinheit zum Thema Mittelwerte gebeten, die wichtigsten Begriffe als SMS schriftlich festzuhalten - also als sehr kurze Nachricht, die die wichtigsten Informationen enthält. Die Aufgabenstellung der Studentin lautete:

\section{Mittelwerte}

Dein Freund/deine Freundin hat in den letzten Mathestunden zum Thema „Mittelwerte“ gefehlt. Schreibe ihm/ihr eine SMS und erkläre ihm/ihr anhand der Beispiele, die ihr im Unterricht behandelt habt, die Begriffe „Median“ und „Arithmetisches Mittel“. Pro Erklärung kannst du bis zu 5 Sätze schreiben.

Die Aufgabenstellung erscheint für eine Sicherungsphase geeignet, weil die Schülerinnen und Schüler beim Schreiben das zuvor im Unterricht behandelte Thema Mittelwerte eigenständig zusammenfassen müssen. Dabei ist zu erwarten, dass für die Schüler*innen 
selbst sowie für die Lehrkraft bzw. Praktikantin sichtbar wird, inwieweit der Lernstoff fachlich und sprachlich schon angeeignet ist (vgl. Leitidee L4). Zugleich können sich, wie die Schülerbeispiele in - Abb. 2.8 zeigen, auch Einblicke in individuelle Einschätzungen in Bezug auf den Lernbereich ergeben („Der Median ist im Prinzip eigentlich ganz leicht.“; „Mein Lieblingsmittelwert ist der arithmetische Mittelwert.“). Um den Schreibprozess zu fokussieren, sind in der Aufgabenstellung einige Anhaltspunkte gegeben etwa der Bezug auf die im Unterricht gegebenen Beispiele sowie zwei für den Themenbereich zentrale Begriffe, die erklärt werden sollen.

- Abb. 2.8 zeigt zwei Schülertexte, die mithilfe der SMS-Methode entstanden sind.

Wie in - Abb. 2.8 exemplarisch gezeigt, führte der vergleichsweise einfache Schreibauftrag der Studentin zu reichhaltigen Lernendenprodukten, die häufig auch umfangreicher waren als die geforderte SMS-Länge von fünf Sätzen pro Erklärung.

Um die Studierenden bei der Auswertung der erhobenen Daten zu unterstützen, wurde im Begleitseminar zum Praxissemester zunächst die Vorstellungsorientierung diskutiert, die in den Lernendenprodukten zum Ausdruck kommt. So verdeutlichten die Schüler die thematisierten mathematischen Begriffe anhand sinnstiftender Beispiele, etwa die Vorstellung des Durchschnitts mit der Schwerpunkteigenschaft (Sill 2016). Dem zugrunde liegt die alltägliche Erfahrung einer Wippe (z. B. auf Spielplätzen), die in ein Gleichgewicht gebracht wird (in Schülerbeispiel 2 als „Waage“ bezeichnet). Die Mitte im Sinne eines Gleichgewichtspunktes ist eine tragfähige Vorstellung des arithmetischen Mittels. Auch das Beispiel zum Median (Schülerbeispiel 1) ist gut gewählt, da sich anhand der Körpergröße wichtige Aspekte des Medianbegriffs verdeutlichen lassen. Dabei gibt der Schüler im Sinne einer Anleitung an, was bei der Ermittlung des Medians zu beachten ist (nämlich die numerische Anordnung der Werte: „Man muss die sortieren“; ,,von Klein nach groß“).

- Abb. 2.8 Lernendenprodukte „Mittelwerte“

\section{Schülerbeispiel 1}

Hi Jonas.

Da du ja gefehlt hast, und dadurch auch den Unterichtsstoff verpasst hast. Erkläre ich dir dann jetzt was, und wie wir das Gelernt haben. Also es gibt einmal den Median der heißt auch Zentralwert, weil er der Wert in der Mitte ist. Der Median ist im Prinzip eigentlich ganz leicht. Den Median bestimmt man so: Erst müssen alle Daten der Größe nach angeordnet. Dann bestimmt man den Wert in der Mitte.

1) Man hat die Daten 3-8-5-9-2-4-11

2) Man muss die sortieren 2-3-4-5-8-9-11

3) Dann bestimmt man den Wert in der Mitte 2-3-4-5 - $8-9-$ 11

4) Also, der Median ist 5

Ich gebe dir ein Beispiel. Alle in der Klasse stellen sich von Klein nach groß auf. Die Körpergröße vom Schüler in der Mitte ist der Median.

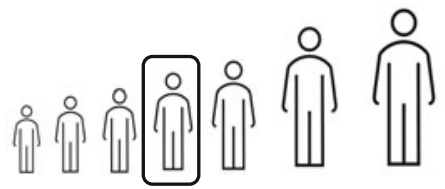


- Abb. 2.8 (Fortsetzung) Begleitveranstaltung thematisiert (dazu $>$ Abschn. 2.3): Die Schüler*innen nutzen nicht nur unterschiedliche (symbolische, ikonische und tabellarische) Darstellungen, sie vernetzen diese auch explizit in ihren Texten (vgl. Schülerbeispiel 2: „Also ist die Anzahl der Werte 5, das sieht man links [in der Tabelle].“; „Auf der Waage sind Gewichte, so wie die Zahlen in der Tabelle.").

Anhand der studentischen Aufgabe konnten im Seminar auch noch einmal explizite Bezüge zu den Kernprozessen hergestellt werden. Zum Beispiel wurde diskutiert, welche weiteren Varianten für eine Sicherungsphase möglich wären oder wie eine Aufgabe verändert werden müsste, um sie in einer Einführungs- oder Vertiefungsphase gewinnbringend einzusetzen. Die vorliegende Aufgabe eignet sich z. B. nicht für eine Einführungsphase, da auf bereits vermittelten Unterrichtsstoff Bezug genommen wird und Fachbegriffe explizit erfragt werden. ${ }^{6}$ Für eine Vertiefungsphase könnte die Aufgabe so abgewandelt werden, dass der Ausbau der mathematischen Kommunikationskompetenz stärker in den Mittelpunkt rückt.

6 Ein Beispiel für einen Schreibauftrag in der Einführungsphase findet sich in $>$ Abschn. 2.6.3.2 (mit Lernendenprodukten in $\mathbf{- A b b}$. 2.19). 


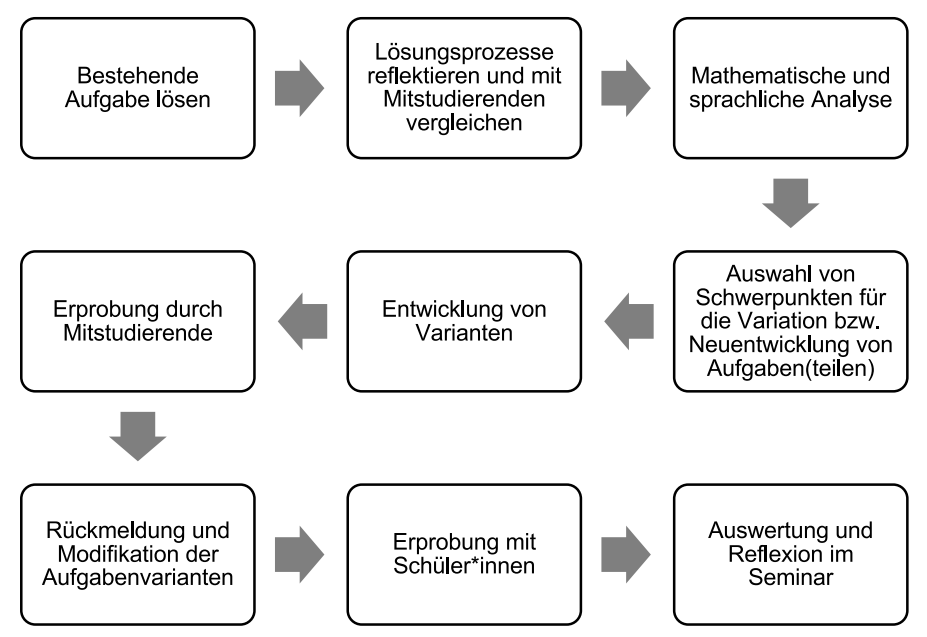

- Abb. 2.9 Prozess der Aufgabenanalyse und -variation

- Abb. 2.9 gibt einen Überblick, wie die Arbeit an Aufgaben unter Berücksichtigung von Aspekten der Sprachbildung in Lehrveranstaltungen gestaltet werden kann. Den Ausgangspunkt bilden bestehende (Schulbuch-)Aufgaben, die die Studierenden zunächst selbst lösen; der Lösungsprozess wird dabei bereits zum Gegenstand der Reflexion gemacht. Vor dem Hintergrund geeigneter theoretischer Ansätze werden dann (z. B. mit Hilfe der Checkliste zur Sprachbildung, - Abb. 2.6) Aufgabenvariationen vorgenommen oder neue Aufgaben(teile) entwickelt, die die Rolle von Sprache im Mathematikunterricht bewusst adressieren. Es bietet sich an, diese Aufgaben innerhalb der Seminargruppe zu erproben und ggf. Modifikationen vorzunehmen, bevor sie beispielsweise im Rahmen eines studentischen Forschungsprojekts Lernenden zur Bearbeitung gegeben werden. Den Abschluss bildet eine Reflexion in der Seminargruppe, in der zentrale Schritte und Hilfen bei der Arbeit an Aufgaben zusammengefasst werden.

\subsection{Darstellungsvernetzungen adressieren}

I) Mir ist aufgefallen, dass die Schüler große Schwierigkeiten hatten, funktionale Zusammenhänge zu erkennen und zu versprachlichen. Ich habe dann mit einem digitalen Werkzeug Aufgaben entwickelt, bei denen sie zwischen ikonischer, tabellarischer und graphischer Darstellung wechseln konnten. Die Schüler haben immer neue schriftliche Vermutungen zu funktionalen Zusammenhängen aufgestellt und dann überprüft - das war schon fast wie ein Wettbewerb! (Studierende, Masterkolloquium 2019). ${ }^{7}$

Darstellungen (und ihre Vernetzung) sind für das Fach Mathematik von großer Bedeutung, weil sie das inhaltliche Denken unterstützen und Kommunikationsanlässe für eine produktive Auseinandersetzung im Unterricht bieten können. Der vorliegende Abschnitt zeigt anhand konkreter Beispiele, wie das Thema Darstellungsvernetzung im Rahmen einer sprachbewussten Hochschullehre thematisiert werden kann. Dabei wird insbesondere

7 Ergebnisse aus der Masterarbeit werden vorgestellt in Schacht et al. (i. V.). 
diskutiert, wie sich Darstellungen für die Variation von Aufgaben ( Abschn. 2.2) nutzen lassen.

Damit werden in diesem Abschnitt sowohl Leitideen zu fachbezogenem Sprachwissen (L1) als auch zur Rolle von Aufgaben bzw. den darin verwendeten Darstellungen (L2) konkretisiert. Besonders eingegangen wird auf die Beurteilung der Rolle von Darstellungen für das fachliche und sprachliche Lernen sowie auf die Nutzung von Darstellungsvernetzungen als Element der Sprachbildung. Hinsichtlich der hochschuldidaktischen Design-Prinzipien wird in diesem Abschnitt die Integration von Sprache und Fach sowohl auf struktureller und planungsbezogener Ebene (DP1) als auch auf inhaltlicher Ebene zur Arbeit mit authentischen Lerngegenständen (DP2) thematisiert.

\subsubsection{Musiker für Schulfest gesucht - Bedingte Wahrscheinlichkeiten}

Für den Aufbau inhaltlichen Denkens im Fach Mathematik ist es zentral, dass Lernende unterschiedliche Darstellungen kennen und diese miteinander vernetzen können. Im sprachbildenden Mathematikunterricht ist damit die Herausforderung verknüpft, die mit den jeweiligen Darstellungen verbundenen Sprachmittel gezielt zu adressieren. Dies kann in der universitären Lehre an unterrichtsrelevanten Beispielen theoretisch fundiert und reflektiert werden. Im Folgenden dient als Ausgangspunkt ein Aufgabenkontext zum Thema „Bedingte Wahrscheinlichkeiten“ (dazu auch Guckelsberger und Schacht 2018, S. 30):

\section{Kontext zu bedingten Wahrscheinlichkeiten}

Für das Schulfest werden noch Musiker gesucht. Die Klasse 9a hat unter den 25 Schülerinnen und Schülern eine Umfrage gemacht. Diese ergab, dass jedes dritte Mädchen ein Instrument spielt, während es bei den Jungen 2 von 10 sind.

Es bietet sich an, die im Kontext „Schulfest“ enthaltenen Informationen zunächst in unterschiedlichen Darstellungsformen zu strukturieren und zu visualisieren (- Abb. 2.10; entnommen aus Guckelsberger und Schacht 2018). ${ }^{8}$ So lässt sich mit Hilfe eines Baumdiagramms die sequenzielle Struktur mehrstufiger Vorgänge abbilden. Hingegen eignet sich das Einheitsquadrat, um die Verhältnisse der zugrunde liegenden Daten zu veranschaulichen. Die Vierfeldertafel wiederum bietet die Möglichkeit, bivariate metrische Daten darzustellen und zu interpretieren: „Mit der Auswertung und Interpretation solcher Vierfeldertafeln im Rahmen von Gruppenvergleichen lassen sich wichtige Grundbegriffe der Stochastik vorbereiten, nämlich die statistische Abhängigkeit und die bedingte Wahrscheinlichkeit" (Krüger et al. 2015, S. 111). Die symbolische Darstellung wird schließlich für die Berechnung von Wahrscheinlichkeiten genutzt. Die Darstellungen machen also jeweils unterschiedliche Spezifika der beschriebenen Situation sichtbar.

- Abb. 2.10 verdeutlicht, dass mit jeder Darstellungsform nicht nur spezifische Begriffsfacetten verknüpft sind, sondern auch jeweils besondere sprachliche Mittel (Wessel 2015; Duval 2006). So lassen sich etwa in der Vierfeldertafel die bedingten Wahrschein-

8 Vgl. Eichler und Vogel (2010) zu Vor- und Nachteilen unterschiedlicher Visualisierungen in der Wahrscheinlichkeitsrechnung. 


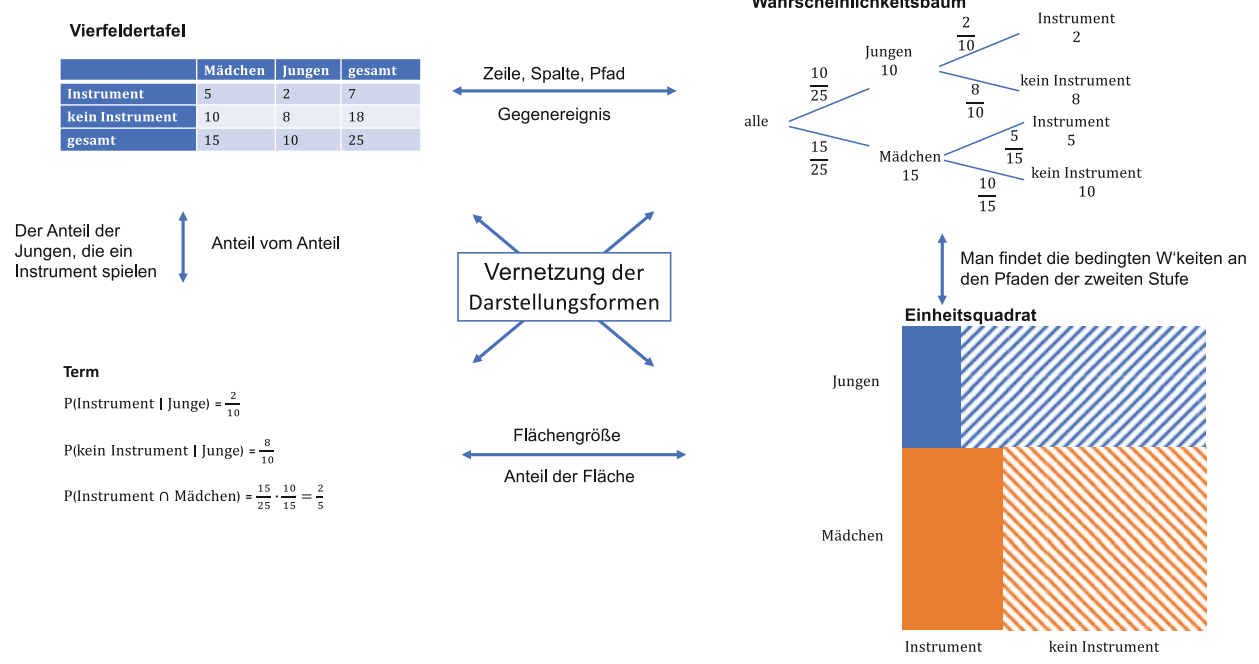

- Abb. 2.10 Darstellungsformen für bedingte Wahrscheinlichkeiten

lichkeiten nicht direkt ablesen. Es ist daher eine zentrale inhaltliche und sprachliche Herausforderung, diese entlang der Werte in den Zeilen und Spalten korrekt zu ermitteln. Inhaltlich werden dafür Anteile von Anteilen bestimmt (dazu auch Schacht et al. 2020). Umgekehrt liefert die sequenzielle Darstellung des Wahrscheinlichkeitsbaums eine gute Übersicht über die Mehrstufigkeit des Zufallsversuchs, wobei eine zentrale semantische Herausforderung darin liegt, die Wahrscheinlichkeiten als Multiplikation der relativen Häufigkeiten entlang der einzelnen Pfade zu interpretieren. Welche besonderen sprachlichen Anforderungen sich daraus jeweils ergeben, wird im nächsten Abschnitt systematisch beschrieben (dazu etwa Prediger 2020; Wessel 2015; Duval 2006; Guckelsberger und Schacht 2018)

\subsubsection{Merkmale des stochastischen Kontexts "Bedingte Wahrscheinlichkeiten"}

Aus mathematischer Perspektive bieten Problemstellungen wie die obige eine reichhaltige Substanz. Die Lernenden können z. B. Anteile bzw. Wahrscheinlichkeiten bestimmen. Weil hierbei mehr als ein Merkmal eine Rolle spielt (Musiker bzw. Nicht-Musiker und Junge bzw. Mädchen), müssen bedingte Wahrscheinlichkeiten bestimmt werden. Von einer bedingten Wahrscheinlichkeit wird z. B. in dem Fall gesprochen, dass $\frac{1}{5}$ der Jungen (im Unterschied zu: $\frac{1}{5}$ aller Befragten) ein Instrument spielt. Der Anteil bezieht sich hier ausschließlich auf die Merkmalsausprägung „Junge“. Folglich beträgt die bedingte Wahrscheinlichkeit dafür, dass ein zufällig gewählter Junge der Klasse ein Instrumentalist ist, $\frac{1}{5}$.

Eine zentrale begriffliche Herausforderung darin liegt, die unterschiedlichen Darstellungen nicht nur einzeln zu betrachten, sondern sie miteinander in Beziehung zu setzen und zu vernetzen (vgl. dazu z. B. auch Duval 2006; Leisen 2005, 2010; sowie Eichler 
und Vogel 2010 als Beispiel zur Wahrscheinlichkeitsrechnung). Welche Potentiale für die Sprachbildung im Fach Mathematik daraus erwachsen, zeigen sowohl Studien mit Lernenden, die lebensweltlich mehrsprachig aufwachsen (u. a. Prediger und Wessel 2011, Wessel 2015), als auch Untersuchungen mit einsprachig aufwachsenden Lernenden. So zeigen Schacht et al. (i. V.) am Beispiel funktionaler Zusammenhänge auf, wie sich durch die Auseinandersetzung mit unterschiedlichen Darstellungsformen und mit Hilfe von digitalen Werkzeugen fachbezogene sprachliche Handlungen gezielt initiieren und fördern lassen. Dies ist umso wichtiger angesichts von Forschungsbefunden, die belegen, dass Lernschwierigkeiten im Fach Mathematik mit Problemen bei der Darstellungsvernetzung einhergehen (Radatz 1991; Moser Opitz 2007). Die Zielperspektive ist daher ein breites inhaltliches Verstehen inklusive der jeweiligen Sprachmittel, die für die einzelnen Darstellungsebenen genutzt werden müssen.

\subsubsection{Sprachbewusste Aufgabenvariation durch Darstellungsvernetzung}

Der o.g. Aufgabenkontext „Schulfest“ kann in der Hochschullehre genutzt werden, um Aufgabenvariationen mit einem Schwerpunkt auf der Vernetzung von Darstellungsformen zu thematisieren. Den Ausgangspunkt bilden folgende Arbeitsaufträge an die Studierenden:

\section{Sprachbildung durch Darstellungsvernetzung}

Nutzen Sie den folgenden Kontext aus dem Lernbereich Bedingte Wahrscheinlichkeiten, um Aufgaben mit einem Schwerpunkt auf Darstellungsvernetzung zu entwickeln:

॥ Für das Schulfest werden noch Musiker gesucht. Die Klasse 9a hat unter den 25 Schülerinnen und Schülern eine Umfrage gemacht. Diese ergab, dass jedes dritte Mädchen ein Instrument spielt, während es bei den Jungen 2 von 10 sind.

Setzen Sie dabei die folgenden Schwerpunkte:

- Übersetzungsprozesse initiieren: Hier sollte der Darstellungswechsel von der einen Darstellung in die andere Darstellung im Vordergrund stehen.

- Vernetzungsprozesse initiieren (vergleichen und vernetzen): Bei dieser Aufgabenvariante sollte die gezielte Vernetzung (also nicht nur der Wechsel) im Fokus stehen.

- Lernen am sprachlichen Vorbild: Erarbeiten Sie eine Variante, bei der sprachliche Vorbilder gezielt genutzt werden.

- Darstellungen und Darstellungswechsel „erleben“: Hier können Sie eine Aufgabenvariante konstruieren, bei der bedingte Wahrscheinlichkeiten praktisch-handelnd erfahren werden können.

Arbeiten Sie für jede der Aufgabenvarianten Potentiale und Herausforderungen für die Umsetzung im Mathematikunterricht heraus. 


\subsubsection{Aufgabenvariante 1: Übersetzungsprozesse initiieren}

Eine erste Möglichkeit, einen Darstellungswechsel gezielt zu initiieren, besteht darin, den Aufgabentext in weitere Darstellungen zu übersetzen. Hierfür sollten Arbeitsaufträge für die Schüler*innen formuliert werden, die die entsprechenden Darstellungsformen konkret benennen:

Für das Schulfest werden noch Musiker gesucht. Die Klasse 9a hat unter den 25 Schülerinnen und Schülern eine Umfrage gemacht. Diese ergab, dass jedes dritte Mädchen ein Instrument spielt, während es bei den Jungen 2 von 10 sind.

- Bilde die Situation in einem Einheitsquadrat ab.

- Erstelle ein Baumdiagramm.

- Stelle die Daten in einer Vierfeldertafel dar.

In diesem Fall besteht die inhaltliche Herausforderung darin, die im Text gegebene Sachsituation in die Darstellungsformen Einheitsquadrat, Baumdiagramm und Vierfeldertafel zu übersetzen. Diese halten jeweils spezifische fachliche und sprachliche Hürden bereit, die im Mathematikunterricht gezielt zu adressieren sind. Sollen die im obigen Text genannten Informationen in ein Einheitsquadrat überführt werden, so müssen zunächst die entsprechenden Merkmale bestimmt werden, in diesem Fall also Junge/Mädchen bzw. Instrument/kein Instrument. Auf der Ebene der Textrezeption ist es daher notwendig, diese Kategorien zu identifizieren bzw. - auf der Ebene der Textproduktion - begrifflich zunächst zu bilden. Dies ist also ein entscheidender Aspekt des zugrundeliegenden fachlichen Begriffsbildungsprozesses der Lernenden, der bei der Planung entsprechender Aufgaben durch die Studierenden berücksichtigt werden sollte. Sind die Kategorien einmal gefunden, ist es nun entscheidend, die jeweiligen Verhältnisse zu bilden. Dies erfordert auf der Ebene der Textrezeption eine genaue Rekonstruktion der zugrunde liegenden Anteile. Typische Schwierigkeiten bestehen in dem obigen Fall etwa darin, dass sowohl Zahlzeichen (,2 [von] 10“) als auch Zahlwörter („,[jedes] dritte [Mädchen]“) verwendet werden, dass absolute Häufigkeiten (,10 Jungen“) und relative Häufigkeiten (,,jedes dritte Mädchen“) genannt werden und dass explizite Angaben (,10 Jungen“) und nicht-explizite Angaben (Anzahl der Mädchen in der Klasse: $25-10=15$ ) enthalten sind. Bei der Rekonstruktion spielt hier insbesondere die Bildung von Anteilen eine zentrale Rolle, dies unter besonderem sprachlichen Fokus der Präposition ,,von“.9 Sind die absoluten Häufigkeiten einmal ermittelt, so müssen die relativen Häufigkeiten bzw. die Anteile gebildet werden. Diese sind notwendig, um die entsprechenden Flächenanteile abzubilden. Schließlich müssen die Flächenstücke korrekt interpretiert werden.

Auch für weitere Übersetzungsprozesse ergeben sich spezifische sprachliche und fachliche Anforderungen (vgl. - Tab. 2.1). So müssen etwa für die Vierfeldertafel zunächst nicht die relativen Häufigkeiten bestimmt werden und damit auch nicht die entsprechenden Flächenanteile, sondern die absoluten Anzahlen. Fachliche und sprachliche Herausforderungen bei der Erstellung der Vierfeldertafel ergeben sich etwa im Zusammenhang mit den Zeilen- und Spaltensummen, die vor dem Hintergrund des Kontextes interpretiert werden müssen.

9 Hier kann mit den Studierenden - bzw. mit den Schülerinnen und Schülern - sprachkontrastiv erarbeitet werden, wie Anteile in anderen Sprachen konzeptualisiert und verbalisiert werden (s. Prediger et al. 2019a). 
- Tab. 2.1 Sprachliche und fachliche Übersetzungsschritte zwischen unterschiedlichen Darstellungen

\begin{tabular}{|c|c|c|c|c|c|c|c|}
\hline $\begin{array}{l}\text { Übersetzungs- } \\
\text { schritt }\end{array}$ & Text & $\rightarrow$ & $\begin{array}{l}\text { absolute } \\
\text { Häufigkeiten }\end{array}$ & $\rightarrow$ & $\begin{array}{l}\text { relative } \\
\text { Häufigkeiten }\end{array}$ & $\rightarrow$ & Einheitsquadrat \\
\hline $\begin{array}{l}\text { sprachliche } \\
\text { und fachliche } \\
\text { Anforderungen }\end{array}$ & \multicolumn{2}{|l|}{$\begin{array}{l}\text { - explizite und nicht } \\
\text { explizite Angaben } \\
\text { - absolute und relati- } \\
\text { ve Häufigkeiten } \\
\text { - Zahlwörter und } \\
\text { Zahlzeichen }\end{array}$} & \multicolumn{2}{|c|}{$\begin{array}{l}\text { - Präpositionen bei } \\
\text { absoluten Häufig- } \\
\text { keiten: } 3 \text { von } 10 \\
\text { - Ganze bestimmen }\end{array}$} & \multicolumn{2}{|c|}{$\begin{array}{l}\text { - Präpositionen bei } \\
\text { Anteilen } \\
\text { - Prozente als Anteile } \\
\text { - Ganze bestimmen }\end{array}$} & $\begin{array}{l}\text { - Flächenanteile } \\
\text { benennen }\end{array}$ \\
\hline
\end{tabular}

Um Darstellungswechsel im Rahmen einer sprachbewussten Lehramtsausbildung zu nutzen, kann es produktiv sein, diese zunächst selbst für die eigenen universitären Lernprozesse zu reflektieren und zu erfahren. Hier bietet es sich an, Beispiele aus universitären Lehrveranstaltungen (z. B. aus fachbezogenen Vorlesungen) auf die Nutzung unterschiedlicher Repräsentationsformen hin zu untersuchen. Für Studierende ist es i. d. R. im Rückblick sehr erhellend, die eigene Begriffsbildung - etwa im Rahmen einer ZahlentheorieVorlesung - zu reflektieren und die Rolle von Darstellungsformen dabei gezielt in den Blick zu nehmen. Neben dem Rückblick ist es im Rahmen der Lehrveranstaltung auch durchaus produktiv, den Studierenden eine fachbezogene Aufgabe zu stellen und diese mit unterschiedlichen Darstellungsformen zu bearbeiten. Das folgende Beispiel zur Gültigkeit der Gaußschen Summenformel etwa ist Gegenstand fast jeder ArithmetikVorlesung. Der Zusatz, die jeweils spezifischen sprachlichen und inhaltlichen Anforderungen der einzelnen Begründungsvarianten zu analysieren, regt dazu an, eigene Lernprozesse (hier: im Rahmen des Studiums) hinsichtlich der Rolle der Sprache gezielt zu reflektieren.

\section{Sprachliche Anforderungen bei Begründungen}

Begründen Sie die Gültigkeit der Gaußschen Summenformel $\sum_{i=1}^{n} i=\frac{n \cdot(n+1)}{2}$ auf unterschiedliche Weise:

a) Begründung mit Hilfe eines generischen Beispiels

b) Begründung mit Hilfe eines generischen Bildes

c) Begründung mit Hilfe eines allgemeinen Bildes

d) Begründung mittels Algebraisierung

e) Begründung mittels vollständiger Induktion

Vergleichen Sie die sprachlichen und inhaltlichen Anforderungen der unterschiedlichen Begründungsvarianten.

\subsubsection{Aufgabenvariante 2: Vernetzungsprozesse initiieren}

Eine zweite Variante besteht darin, nicht allein die Übersetzungsprozesse zum Thema zu machen, sondern Darstellungen gezielt miteinander zu vernetzen und diese Vernetzung selbst zum Reflexionsgegenstand zu machen. Dies kann etwa durch die folgenden Aufgabenstellungen erreicht werden (- Abb. 2.11). 
In der Klasse 9b wurde ebenfalls eine Erhebung durchgeführt.

Das Ergebnis ist in den drei abgebildeten Darstellungsformen zu sehen.

\section{Einheitsquadrat}

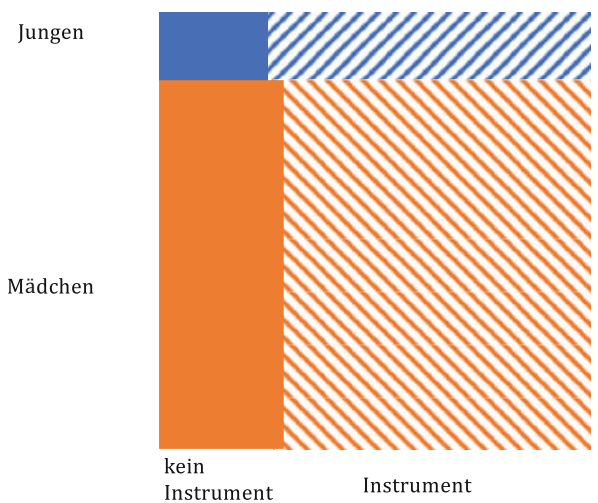

\section{Wahrscheinlichkeitsbaum}

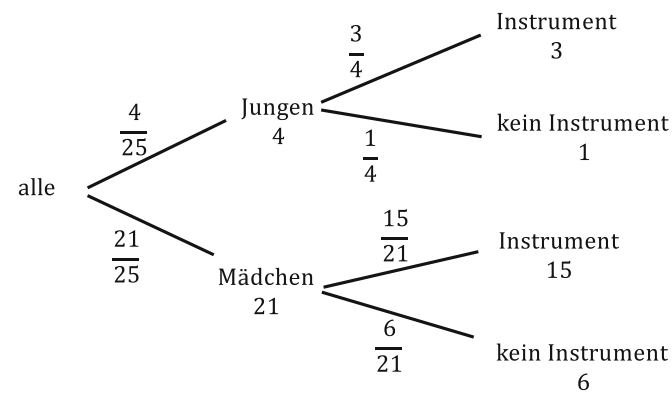

\section{Vierfeldertafel}

\begin{tabular}{|l|l|l|l|}
\hline & Mädchen & Jungen & gesamt \\
\hline Instrument & 15 & 3 & 18 \\
\hline kein Instrument & 6 & 1 & 7 \\
\hline gesamt & 21 & 4 & 25 \\
\hline
\end{tabular}

Erkläre, wie man die folgenden Aussagen jeweils mit den drei Darstellungsformen verdeutlichen kann:

- 3 von 4 Jungen spielen ein Instrument

- fast alle Instrumentalisten sind weiblich

- fast alle Jungen spielen ein Instrument

- $(\ldots)$

Vergleiche die Darstellungen.

Benenne je 2 Aspekte, die in allen Darstellungen gleichermaßen dargestellt sind.

Findest du für jede Darstellung Aspekte, die nur dort deutlich werden?

- Abb. 2.11 Aufgabe zur Vernetzung von Darstellungsformen 
Im Rahmen der Aufträge in - Abb. 2.11 steht die Sichtbarmachung einzelner Aussagen in den jeweiligen Darstellungen im Vordergrund. Die Kraft einer solchen Vernetzung besteht darin, dass die gleiche Aussage in unterschiedlichen Darstellungen - und damit auch mit unterschiedlichen mathematischen und sprachlichen Mitteln - verdeutlicht wird. So lässt sich am Einheitsquadrat etwa sehr übersichtlich ablesen, dass fast alle Instrumentalisten weiblich sind, wenn man die beiden rechten Zellen (der Instrumentalisten) betrachtet. Dies lässt sich mit Zahlen sowohl absolut über die Vierfeldertafel (15 Mädchen und 3 Jungen sind Instrumentalisten) als auch über das Baumdiagramm mit Hilfe relativer Häufigkeiten ( $\frac{3}{4}$ der Jungen spielen ein Instrument) begründen.

\subsubsection{Aufgabenvariante 3: Lernen am sprachlichen Vorbild}

Die Rolle von sprachlichen Vorbildern ist zentral für gelingende Sprachbildung im Fachunterricht. Mit den folgenden Aufgabenstellungen werden sprachliche Mittel bereitgestellt, die die Schülerinnen und Schüler den entsprechenden Darstellungen zuordnen sollen.

Ordne die Sätze einer der Darstellungen zu. Begründe deine Entscheidung.

- „Mehrstufige Zufallsversuche lassen sich in dieser Darstellung besonders gut darstellen.“

- „Auf den Pfaden werden Wahrscheinlichkeiten abgetragen.“

- „Diese Darstellung eignet sich deshalb besonders gut, weil man direkt kontrollieren kann, ob die Zahlen stimmen.“

Anders als bei den Aufgabenvarianten 1 und 2 werden bei dieser Variante Sprachbeispiele genutzt, um die Eigenschaften der jeweiligen Darstellungsformen gezielt gegenüberzustellen. Dabei wird ein qualitativer Vergleich der Darstellungen selbst vorgenommen. Dies ist aus fachlicher Sicht zentral, denn für einen tragfähigen Vorstellungsaufbau ist es wichtig, sich auch mit den Grenzen und Potentialen der jeweiligen Darstellungsform auseinanderzusetzen. Eine sprachliche Anforderung besteht in den obigen Beispielsätzen darin, diese hinsichtlich der genutzten Fachsprache zu dechiffrieren (z. B. ,Wahrscheinlichkeiten abtragen"). Die Aussagen dienen umgekehrt als sprachliches und fachliches Modell, um ähnliche Aussagen selbst aufzustellen und diese mit Hilfe der Darstellungen zu begründen.

\subsubsection{Aufgabenvariante 4: Darstellungen beurteilen}

Eine besondere Kompetenz besteht darin, verschiedene Darstellungsformen selbstständig zu beurteilen. In - Abb. 2.12 ist ein möglicher Schreibauftrag abgedruckt: Die Schüler*innen sollen begründen, warum ihnen die Arbeit mit bestimmten Darstellungen leichter fällt als mit anderen. Die zugehörigen Schülerbearbeitungen zeigen, dass ein solcher Schreibauftrag zur fachlichen Reflexion anregt, weil die Schüler*innen die Darstellungen dabei auch noch einmal miteinander in Beziehung setzen.

Die Schülerantworten machen deutlich, dass Lernende durchaus in der Lage sind, die jeweiligen Vorteile der einzelnen Darstellungen zu benennen. So argumentiert etwa ein Schüler, dass sich im doppelten Baumdiagramm die relativen Häufigkeiten und die „Stufen“ (gemeint ist hier die sequenzielle Struktur) gut ablesen lassen, während in einem anderen Lernertext hervorgehoben wird, dass bei Termen „konkret dargestellt ist, was gesucht/gefordert ist" ( Abb. 2.12). 
Aufgabe: Finde für jede Aussage mindestens zwei Argumente.

Begründe, mit welcher Darstellungsform du am besten arbeiten kannst.

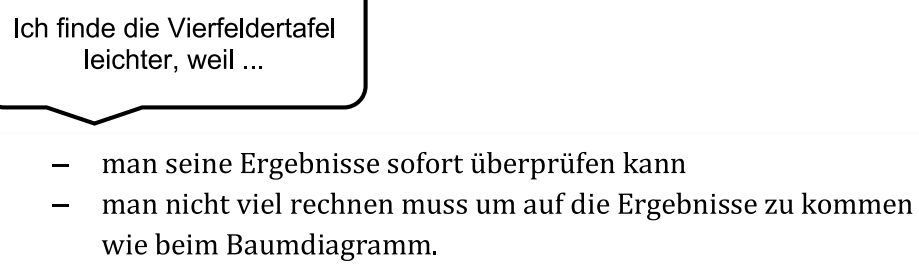

- man seine Ergebnisse sofort überprüfen kann

- man nicht viel rechnen muss um auf die Ergebnisse zu kommen wie beim Baumdiagramm.

Ich finde das doppelte

Baumdiagramm leichter, weil ...

- relative Häufigkeiten ablesbar sind

- Stufen sind gut ablesbar

z.B. 3 von 4 Jungen spielen ein Instrument

$=>$ Pfad $\frac{4}{25}$ Jungen anschauen, dann den Pfad zum Instrument hin $=>\frac{3}{4}$

Ich kann mit Termen

besser arbeiten, weil ...

- es in einem Term konkret dargestellt ist, was gesucht/gefordert ist

- Abb. 2.12 Schreibauftrag und Schülerdokumente zur Beurteilung von Darstellungsformen

\subsubsection{Aufgabenvariante 5: Darstellungen und Darstellungswechsel "erleben"}

Lebendig wird eine Darstellung mathematischer Zusammenhänge, wenn man diese experimentell nachstellt bzw. die Merkmale und Eigenschaften nutzt, um sie entlang der Gruppenmitglieder (an der Schule oder Hochschule) real abzubilden. So kann eine Situation wie die im Kontext „Schulfest“ skizzierte ( Abschn. 2.3.1) genutzt werden, um Schüler*innen oder auch Studierende um eine möglichst übersichtliche Aufstellung im Raum zu bitten. Hierbei sollte zunächst ein möglichst offener Arbeitsauftrag gestellt werden, ohne vorab die Struktur - etwa die der Vierfeldertafel - vorzugeben. Dies erscheint für den Arbeitsprozess deutlich produktiver, weil die Teilnehmenden unterschiedliche Strukturierungsvarianten selbst auswählen können und sich argumentativ der übersichtlichsten Variante nähern.

Gerade das körperliche Erleben von Darstellungsformen kann in der universitären Ausbildung geeignet sein, um den gewohnten Blick auf Darstellungen und deren Rol- 
- Abb. 2.13 Aufstellung und von den Studierenden erstelltes Tafelbild

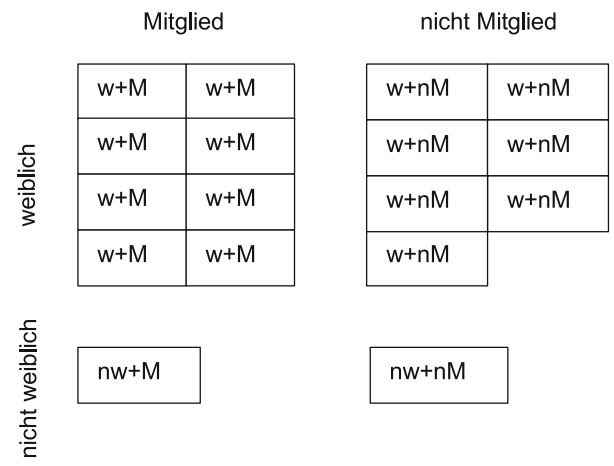

\begin{tabular}{c|c|c|c} 
& $\mathrm{M}$ & $\overline{\mathrm{M}}$ & \\
\hline $\mathrm{W}$ & $\frac{8}{15}=\frac{16}{30}$ & $\frac{7}{15}=\frac{14}{30}$ & 1 \\
\hline$\overline{\mathrm{W}}$ & $\frac{1}{2}=\frac{15}{30}$ & $\frac{1}{2}=\frac{15}{30}$ & 1 \\
\hline & & & $\begin{array}{c}2 \\
(200 \%)\end{array}$
\end{tabular}

le im Mathematikunterricht noch einmal zu verändern. So stellte in einem Seminar eine Studierende die Hypothese auf, dass der Anteil der Männer, die Mitglied im Sportverein sind, größer ist als der Anteil der Frauen, die Mitglied im Sportverein sind. Die Studierenden wurden gebeten, sich dieser Hypothese so anzunähern, dass sie sie durch eine möglichst übersichtliche Anordnung im Raum verifizieren. Alle Studierenden waren zu dem Zeitpunkt bereits gut vertraut mit den entsprechenden mathematischen Darstellungsformen. Die Aufstellung der Studierenden im Seminarraum war schnell gefunden; sie ist in - Abb. 2.13 (oben) wiedergegeben.

Es waren also von den 17 anwesenden Studierenden 15 weiblich und zwei männlich; insgesamt waren 9 Personen Mitglied und 8 Personen kein Mitglied in einem Sportverein. Einen Diskussionspunkt bei der Übertragung dieser Aufstellung in eine geeignete Darstellung (- Abb. 2.13, unten) bildete zunächst die Frage der Benennung der Merkmale. Dabei spielt es aus fachlicher Perspektive natürlich überhaupt keine Rolle, ob etwa „w“ für ,,weiblich“ und „,m“ für „männlich“ gewählt wird. Erst aus der Rückschau erscheint es sinnvoll, Bezeichner zu wählen, die einen Zusammenhang zwischen den jeweiligen Merkmalsausprägungen erkennen lassen, wie in der Tafelanschrift oben „ $W$ “ für „,weiblich“ und , $\bar{W}$ “ für ,nicht weiblich“. Gerade im Umgang mit Termen (etwa bei der Bestimmung von Wahrscheinlichkeiten für die Gegenereignisse) ist es übersichtlich(er), die Bezeichnungen entsprechend zu wählen. Dass dies für Schüler*innen im Mathematikunterricht keine selbstverständliche Erfahrung ist, sondern eine, die erst erlernt und dann aus der Rückschau reflektiert und eingeordnet werden muss, ist für Studierende eine wichtige Einsicht.

Wie das Tafelbild zeigt, führte der Versuch der Studierenden, ihre Aufstellung im Seminarraum in eine Vieferfeldertafel zu überführen, zu einem interessanten Fehler: Es wurden anstelle der absoluten Häufigkeiten fälschlicherweise die jeweiligen bedingten Wahrscheinlichkeiten zugrunde gelegt (- Abb. 2.13, unten). Die Studierenden haben also eine „Vierfeldertafel“ entwickelt, die die jeweiligen Anteile einer Merkmalsausprägung 
aufführte. In der Aufstellung (- Abb. 2.13, oben) hatte sich gezeigt, dass 8 von 15 weiblichen Studierenden Mitglied eines Sportvereins sind, 7 von 15 hingegen nicht. Genau diese Verhältnisse wurden vor dem Hintergrund der Aufstellung in der Vierfeldertafel abgetragen. Gleiches passierte bei den männlichen Studierenden: Jeweils die Hälfte der anwesenden Studenten war Mitglied bzw. Nicht-Mitglied eines Sportvereins. Obwohl die Studierenden thematisch bereits eingearbeitet waren, sorgte auch die „Kontrolle“ (Addition der jeweiligen Anteile zu 200\% statt 100\%) zunächst nicht für einen kognitiven Konflikt. Erst als eine Studentin die Darstellung hinterfragte, entwickelte sich eine produktive Diskussion, in der die Vierfeldertafel überarbeitet und korrigiert wurde.

Das Beispiel führt anschaulich vor Augen, wie erfahrungsbezogenes Lernen auch auf Hochschulebene zu einer intensiven inhaltlichen Auseinandersetzung - in diesem Fall über den Aufbau und die Struktur einer Darstellungsform - führen und dadurch vermeintliche mathematische Selbstverständlichkeiten und gewohnte Schemata aufbrechen kann.

\subsection{Empirische Daten aus dem Unterricht als Analyse- und Lerngegenstand}

॥ Ich habe einem Schüler und einer Schülerin der 8. Klasse Blütenaufgaben zu linearen Gleichungen vorgelegt, die immer schwieriger wurden. Die beiden haben sich bei der gemeinsamen Bearbeitung selbst mit dem Smartphone aufgenommen und ich habe das im Anschluss transkribiert. Dann habe ich analysiert, wie sie bei der Lösung der Aufgabe vorgegangen sind und welche sprachlichen und mathematischen Probleme sie hatten. (...) Mir ist bewusst geworden, wie sprachintensiv Mathematik eigentlich ist (Studentin, Praxissemester 2018).

Die universitäre Vorbereitung auf die spätere Schulpraxis sollte die Lernendenperspektive konsequent mitberücksichtigen. Interessante Analyse- und Lerngegenstände bilden in diesem Zusammenhang empirische Daten aus dem Fachunterricht: Anhand von schriftlichen Schülerdokumenten oder (transkribierten) Aufzeichnungen mündlicher Kommunikation lassen sich beispielsweise individuelle Vorgehensweisen, Denkwege und Begriffsvorstellungen von Schülerinnen und Schülern rekonstruieren oder Verständnisschwierigkeiten diagnostizieren. Im Folgenden wird aufgezeigt, wie solche Analyseprozesse in der Hochschullehre initiiert und für die Reflexion von fachlichem und sprachlichem Lernen produktiv genutzt werden können.

Im Vordergrund steht somit die Leitidee L4 zum fachlich-sprachlichen Diagnostizieren mit dem Ziel, das mathematische Verständnis von Lernenden einzuschätzen und entsprechende Konsequenzen für das unterrichtliche Handeln im Sinne eines sprachbewussten Fachunterrichts abzuleiten. Hinsichtlich der hochschuldidaktischen DesignPrinzipien wird in diesem Abschnitt die Integration von Sprache und Fach insbesondere auf inhaltlicher Ebene zur Arbeit mit authentischen Lerngegenständen (DP2) thematisiert: Die Studierenden sollen empirischen Beispielen gegenüber eine forschende Haltung einnehmen; insbesondere sollen sie theoretische Konstrukte zur Analyse sprachlicher und mathematischer Prozesse kennen und diese anwenden, um z. B. die Begriffsbildung, den Umgang mit Darstellungen oder die Lernstände der Schülerinnen und Schüler genauer zu verstehen. 


\subsubsection{Schriftliche und mündliche Unterrichtsdaten in universitären Veranstaltungen}

Für die studentischen Lernprozesse im Rahmen der universitären Lehramtsausbildung ist die Arbeit mit authentischen Produkten von Schülerinnen und Schülern sehr wichtig - nicht nur, um auf die spätere Praxis vorzubereiten, sondern auch, um einen Raum $\mathrm{zu}$ schaffen, der eine theoriegeleitete und bewusst sehr detaillierte Auseinandersetzung mit Lernendenprodukten und -prozessen ermöglicht, bevor fachdidaktisch und pädagogisch begründete Entscheidungen in der späteren beruflichen Praxis häufig unter Zeitdruck und ad hoc gefällt werden (müssen).

Grundsätzlich eignen sich fast alle unterrichtsbezogenen Produkte, um sie einer fachlichen und sprachlichen Analyse zu unterziehen (Caspari 2016). Häufig lohnt sich ein Start mit bereits vorliegenden Schülerdokumenten, die in bisherigen Lehrveranstaltungen eher unter fachlichen Gesichtspunkten untersucht wurden. Wenn solche Daten nicht zur Verfügung stehen, können (z. B. im Rahmen von Seminaren, die an eine Praxisphase gekoppelt sind) vor allem schriftliche Materialien unkompliziert eingeholt werden (natürlich unter Einhaltung der Datenschutzregeln). So könnten die Schüler*innen zum Beispiel gebeten werden, einen fachlichen Zusammenhang in einem Brief an einen Mitschüler (Maier und Schweiger 1999; Abschn. 2.4.3) oder auf einem „Spickzettel“ (Prediger 2003) zu erläutern und dabei Abbildungen, Tabellen oder sonstige darstellerische Mittel zu nutzen.

Bei der Einbindung empirischer Daten aus dem Unterricht in die Hochschullehre ist Folgendes zu berücksichtigen:

- Genaue sprachliche Auseinandersetzung: Um sich den empirischen Daten zu nähern, ist zunächst die sehr genaue Lektüre der schriftlichen oder transkribierten mündlichen Äußerungen notwendig. Dabei sollten die Studierenden zu einer Auseinandersetzung angeleitet werden, die fachlich relevante sprachliche Aspekte fokussiert. Dies ist wichtig, weil bei einer ersten Durchsicht schriftlicher Schülerdaten oft vor allem Abweichungen von der grammatischen und orthographischen Norm auffallen; beim Lesen von Transkripten wiederum springen ,unvollständige“ Äußerungen, Reformulierungen und andere Merkmale mündlicher Kommunikation ins Auge. Die Studierenden sollten also lernen, mündliche und schriftliche Äußerungen gezielt auf ihre fachliche und fachsprachliche Qualität hin zu lesen und zu prüfen, um daraus dann Erkenntnisse für entsprechende Unterstützungsmaßnahmen zu ziehen.

- Fachliche Durchdringung: Eine weitere Voraussetzung für eine angemessene Einordnung mündlicher oder schriftlicher Lernendenprodukte ist die fachliche Auseinandersetzung mit den mathematischen Gegenständen. Die Studierenden sollten also beispielsweise zugrunde liegende Aufgaben zunächst selbst lösen.

- Theoriebezug bei der empirischen Analyse: Der konsequente Theoriebezug ist für die Betrachtung der empirischen Daten aus wissenschaftlicher Sicht nicht nur selbstverständlich, er ermöglicht es den Studierenden auch, zu vergleichbaren - wenngleich im Einzelfall durchaus sehr unterschiedlichen - Ergebnissen zu gelangen, die dann im Rahmen der Lehrveranstaltung diskutiert werden können. Im Rahmen des vorliegenden Kapitels werden Anregungen gegeben, inwiefern z. B. die Theorie des Conceptual Change (Duit 1996) eine theoretische Fundierung bietet, um die Entwicklung und Nutzung von Alltags- und Fachbegriffen bei Schülerinnen und Schülern zu untersuchen. 


\subsubsection{Unterrichtsdiskurs zum Abhängigkeitsbegriff als Lerngegenstand}

Anhand des ersten Beispiels wird veranschaulicht, wie Studierende in Lehrveranstaltungen über die analytische Auseinandersetzung mit empirischen Daten - hier einem Transkriptausschnitt aus dem Unterricht einer Jahrgangsstufe 10 zum Thema Stochastik - zum Nachdenken über schülerseitige Begriffsbildungsprozesse angeregt werden können (vgl. Kap. 1; Maier und Schweiger 1999).

Für das Erlernen vieler naturwissenschaftlicher und mathematischer Begriffe bedarf es eines Wechsels von der alläglichen hin zu einer fachlichen Sichtweise (etwa auf den Begriff des Vierecks). Häufig müssen alltägliche Vorstellungen entsprechender Konzepte erweitert, neu geordnet bzw. angepasst oder geändert werden. Duit (1996) u. a. sprechen in Bezug auf physikalisch-mathematische Zusammenhänge von einem Conceptual Change, bei dem es darum geht, neben dem alltäglichen Begriffsverständnis auch das oft abweichende - fachbezogene Verständnis entsprechender Konzepte zu thematisieren. Lernen bedeutet in diesem Sinne

॥ in aller Regel „Umlernen“ (...), da vorunterrichtliche Vorstellungen und naturwissenschaftliche Vorstellungen zumindest in wesentlichen Aspekten konträr gegenüberstehen. Sie sind in unterschiedliche Rahmenvorstellungen eingebettet, Lernen naturwissenschaftlicher Begriffe und Prinzipien erfordert auch den Wechsel dieser Rahmenvorstellungen (Duit 1996, S. 158).

Auch für den Mathematikunterricht folgt daraus, dass es geeigneter Kontexte bedarf, die einen solchen Konzeptwechsel begünstigen bzw. ermöglichen können (vgl. Schnell 2014; Prediger 2008).

Ganz ähnlich wird dies aus sprachbezogener Perspektive auch für andere fachliche Domänen beschrieben. So werden beispielsweise im Fach Geschichte

॥ viele Phänomene mit Begriffen der Alltagssprache erfasst, deren fachsprachliche Verwendung nicht nur eine semantische Umdeutung, sondern vielfach auch ein kontextbezogenes Umdenken der Begriffe erfordert (Roll et al. 2019b, S. 26f.).

Für den unterrichtlichen Alltag bedeutet dies allerdings gerade nicht, das alltägliche Begriffsverständnis durch ein fachliches zu ersetzen. Vielmehr geht es um ein Nebeneinander der Begriffe sowie die bewusste Verwendung je nach Situation:

I) Conceptual change does not imply that initial conceptions are „extinguished“. Initial conceptions, especially those that hold explanatory power in nonscientific contexts, may be held concurrently with new conceptions. Successful students learn to utilize different conceptions in appropriate contexts. That is, the status of one particular conception may change in differing contexts (Tyson et al. 1997, S. 402).

Ein solcher Conceptual Change zwischen alltags- und fachbezogenen Begriffsverwendungen ist für mathematische Konzepte ein typisches Phänomen.

Um die Studierenden zu einer fachlichen und sprachlichen Auseinandersetzung anzuleiten, kann mit einem Transkript wie dem folgenden gearbeitet werden. Das Transkript dokumentiert einen kurzen Auszug aus einem Unterrichtsdiskurs in einer 10. Klasse im 
Fach Mathematik. ${ }^{10}$ Dem Ausschnitt ist die Bearbeitung einer Aufgabe vorangegangen, in der es darum ging, den Anteil von Rauchern und Nicht-Rauchern in einem Sportverein genauer zu untersuchen und entsprechende Wahrscheinlichkeiten zu berechnen. In diesem Zusammenhang wurden auch bedingte Wahrscheinlichkeiten untersucht.

Im Unterrichtsdiskurs wird der Begriff der Abhängigkeit thematisiert. Der Lehrer (L) fragt die Schülerinnen und Schüler, was der Begriff ,,abhängig“ für sie bedeutet.

(s01) S1: Ich würde zum Beispiel

(s02) Also ich bin jetzt zum Beispiel von der Schule abhängig.

(s03) Nein, die Zukunft hängt von der Schule ab.

(s04) L: Aha, und jetzt bezogen auf Sportverein und Rauchen, was würde das bedeuten, ,Abhängigkeit“?

(s05) S1: Ob ich in einem Sportverein spielen darf und ob ich rauche oder ob ich nicht rauche.

(s06) L: Nein.

(s07) S1: Also das hängt davon ab, ob ich in einem Sportverein rauche.

(s08) L: Nein, das steht da erstmal nicht.

(s09) S2: Also ob das von den anderen abhängig ist,

(s10) ob alle, die jetzt Mitglied im Sportverein sind, auch gleichzeitig Raucher sind.

(s11) L: Aha, genau

(s12) also ob da ein Zusammenhang besteht.

(s13) Ich bin im Sportverein.

(s14) Ist es dann z. B. wahrscheinlicher, dass ich rauche oder ist es weniger wahrscheinlich? Was würde man vermuten?

Im Seminar kann das Transkript mithilfe der folgenden Arbeitsaufträge untersucht werden, die insbesondere die Spezifität von alltäglichem und fachbezogenem inhaltlichen Denken adressieren:

\section{Auftrag für Studierende zur Analyse des obigen Transkripts}

Analysieren Sie das Transkript „Abhängigkeit“ aus dem Unterricht einer 10. Klasse. Thematisch geht es in dem Unterrichtsgespräch - hier zwischen dem Lehrer und den beiden Schülern S1 und S2 - um die Frage, was genau unter dem Begriff der Abhängigkeit zu verstehen sei.

- Arbeiten Sie die thematisierten Kontexte für jede Zeile heraus.

- Was lässt sich vor dem Hintergrund des Transkripts über alltags- und fachbezogene Aspekte des Abhängigkeitsbegriffs sagen?

- Welche Impulse würden Sie als Lehrkraft setzen, wenn Sie den Fachbegriff thematisieren möchten und dabei an die Vorerfahrungen der Lernenden anknüpfen?

- Transfer Conceptual Change: Finden Sie weitere Beispiele aus dem Mathematikunterricht, bei denen ein Conceptual Change notwendig ist.

Anhand des Transkripts kann analytisch nachvollzogen werden, welche Alltagskonzepte Schülerinnen und Schüler assoziieren (können), wenn sie über Begriffe sprechen, die,

10 Erhoben von Kathrin Schulze Osthoff (2017) im Rahmen ihrer Masterarbeit. 
wie der Begriff der „Abhängigkeit“, in der Alltagssprache und in der Fachsprache vorkommen. So erfolgt der erste Zugriff in (s02)-(s03) zunächst über für den Schüler S1 relevante Alltagssituationen. Die weiteren Formulierungen in (s07)-(s10) beziehen sich zwar auf den Kontext der Aufgabe, jedoch wird dabei umso deutlicher, wie schwer den Schülern die Annäherung an den Begriff der Abhängigkeit im Inhaltsbereich „Bedingte Wahrscheinlichkeiten" fällt:

- „Ich bin von der Schule abhängig“ (s02)

- „Die Zukunft hängt von der Schule ab“ (s03)

- „Das hängt davon ab, ob ich in einem Sportverein rauche“ (s07)

- „Ob das von den anderen abhängig ist“" (s09)

- „ob alle, die Mitglied im Sportverein sind, auch gleichzeitig Raucher sind“ (s10)

Es zeigt sich, dass allen Schüleräußerungen ein alltägliches Verständnis des Abhängigkeitsbegriffs zugrunde liegt, das durch die Struktur „A hängt von B ab“ gewissermaßen monodirektional, in eine Richtung gehend, geprägt ist. „A“ steht in dieser Struktur für die Person oder die Sache, die abhängig ist (,ich“, „die Zukunft“), „B“ für die Person oder Sache, gegenüber der die Abhängigkeit besteht (,Schule“, „die anderen“). Lediglich die letzte Äußerung ,,[das hängt davon ab,] ob alle, die jetzt Mitglied im Sportverein sind, auch gleichzeitig Raucher sind“ (s10) führt, wenn auch in einer der Mündlichkeit geschuldeten etwas undurchsichtigen Konstruktion, ansatzweise in Richtung eines mathematischen Abhängigkeitsbegriffs, indem Schüler S2 einen Zusammenhang zwischen der Mitgliedschaft im Sportverein und dem Rauchen andeutet; dies greift der Lehrer dann in (s11)-(s12) auch direkt auf.

Bei der stochastischen (Un-)Abhängigkeit handelt es sich gegenüber dem alltäglichen Begriffsverständnis um ein grundverschiedenes Konzept, bei dem Wahrscheinlichkeitsmaße und Ereignisse abstrakt charakterisiert werden:

I) Zwei Ereignisse heißen stochastisch unabhängig, wenn gilt $P(A) \cdot P(B)=P(A \cap B)$. Sonst heißen $A$ und $B$ stochastisch abhängig (Büchter und Henn 2007, S. 205, Hervorhebung im Original).

Auf Unterrichtsebene macht das Beispiel jedenfalls deutlich, wie divergent fachliche und alltägliche Begriffsverwendungen sein können, und es konkretisiert die o.g. Notwendigkeiten für einen Conceptual Change (z. B. Duit 1996; Tyson et al. 1997) bzw. für ein „kontextbezogenes Umdenken der Begriffe“ (Roll et al. 2019b). Die Lernenden sollen also ein inhaltliches Verständnis für den Begriff aus fachinhaltlicher Perspektive entwickeln, ohne dabei jedoch den Alltagsbegriff durch den Fachbegriff zu ersetzen. Im Gegenteil: Wichtig ist gerade, dass Lernende ein Gespür dafür entwickeln, wann ein fachbezogenes Begriffsverständnis (i. S. stochastischer (Un-)Abhängigkeit) und wann ein alltagsbezogenes Begriffsverständnis aktiviert werden muss.

Das Eingangsbeispiel bildet einen guten Anlass, um in der Hochschullehre mit den Studierenden über den stochastischen (Un-)Abhängigkeitsbegriff im Speziellen bzw. mathematische Begriffe im Allgemeinen hinsichtlich ihrer Alltags- bzw. Fachverwendung zu sprechen. So ist es eine durchaus produktive Übung, Situationen zu finden und fachlich und sprachlich zu analysieren, in denen etwa der Begriff der Abhängigkeit oder der Erwartung (,Erwartungswert“") jeweils fachsprachlich bzw. alltagssprachlich genutzt wird; in einem zweiten Schritt können dann entsprechende Impulse oder Aufgaben für den Unterricht entwickelt werden, die die jeweiligen Bedeutungen adressieren. 


\subsubsection{Schülertext zum Thema „Wahrscheinlichkeiten" als Lerngegenstand}

Auch das zweite Beispiel - ein schriftliches Dokument eines neu zugewanderten Schülers aus der oben erwähnten 10. Klasse zum Thema „Wahrscheinlichkeit“ - bietet zahlreiche Anknüpfungspunkte, um im Seminar Zusammenhänge zwischen fachlichem und sprachlichem Lernen zu erschließen.

\section{Beispiel}

Das Schülerdokument in $\mathbf{0}$ Abb. 2.14 ist im Rahmen einer Unterrichtsreihe zum Thema „Bedingte Wahrscheinlichkeiten“ in der Jahrgangsstufe 10 entstanden. Der Arbeitsauftrag für die Schüler*innen lautete: „Ein Mitschüler hat krankheitsbedingt leider das aktuelle Mathethema zur Stochastik verpasst. Schreibt ihm einen Brief, in welchem ihr ihm das Thema erklärt.“

Das Dokument stammt von einem Schüler, der zum Zeitpunkt der Aufgabenbearbeitung seit zwei Jahren in Deutschland (und davor in Syrien) lebte und seitdem die deutsche Sprache erlernt.

Lesen Sie das Schülerdokument in Abb. 2.14 genau und analysieren Sie es hinsichtlich des Zusammenhangs von sprachlichen und fachlichen Aspekten.

Orientieren Sie sich an folgenden Leitfragen:

a) Was erfragt der Schüler mit den von ihm selbst formulierten Aufgaben a) und b) (Z. 06-08)?

b) An welchen Stellen lässt das Schriftprodukt auf einen sicheren bzw. noch nicht sicheren Umgang mit dem Themengebiet schließen?

c) Was lässt sich hinsichtlich des Umgangs mit Darstellungen sagen?

d) Zur weiterführenden Diskussion: Wie könnten die aus der Analyse des Dokuments gewonnenen Erkenntnisse in den Unterricht oder in eine persönliche Rückmeldung einfließen?

Betrachtet man das Beispiel in - Abb. 2.14, so fällt zunächst die gute sprachliche Leistung auf, mit der der Schüler, der zu diesem Zeitpunkt erst seit kurzer Zeit die deutsche Sprache erlernt, das Dokument verfasst hat. Auf Unterrichtsebene stellen Schreibanlässe wie diese eine produktive Möglichkeit dar, um die Schülerinnen und Schüler die Lerngegenstände reflektieren und für sich ordnen zu lassen (vgl. Leitidee 2-3). Dabei soll, so Maier und Schweiger (1999),

॥ die sprachliche Formulierung nicht durch das Befolgen vorgegebener oder erlernter Darstellungsnormen geprägt sein, sondern die Schüler müssen sich eigenständig der ihnen aktiv verfügbaren Sprachmittel bedienen. Schließlich sollte der produzierte Text nicht so sehr an einen Experten (den Lehrer) adressiert sein (...). Stattdessen müssten sich die Schüler als Adressaten einen „Unwissenden“ vorstellen, für den die Problemlösung oder der geschilderte Sachverhalt neu ist und der daher explizit, ausführlich und in verständlicher Sprache informiert werden muss (Maier und Schweiger 1999, S. 148).

Solche Formen der schriftlichen Reflexion im Mathematikunterricht eignen sich vor allem dann, wenn die Lernenden bereits erste Erfahrungen mit den unterrichtlichen Gegenständen haben, also z. B. in einer Sicherungs- oder Vertiefungsphase (vgl. - Abschn. 1.1.1). Im hier diskutierten Schreibkontext steht also die schülerseitige Reflexion im Vordergrund, durch die sich zugleich kompetenzdiagnostische Auskünfte gewinnen lassen. Hingegen geht es nicht um die Vermittlung mathematischer Textsortenkompetenz. Dafür wären andere Schreibaufträge und Herangehensweisen sinnvoll, etwa 
01 Lieber Ömer

02 Ich habe dir das Brief geschrieben um dir zuerklären wie geht mit

03 dem Wahrscheinlichkeit.

04 Wahrscheinlichkeit ist z.B. Wir haben autos die Rot sind und die

05 nicht rot sind und wir haben schüler die volljährig und die

06 nicht volljärig sind, a) wie Wahrscheinlich dass der volljähriger

07 Schüler ein rotes auto besitzt und b) die nicht volljähriger

08 Schüler die Kein rotes auto besitzen.

09 Volljährige $75 \%$ besetzen wir für volljährig $V$

10 nicht Volljährige $25 \%$ “ “ " nicht “ $\bar{V}$

11 rotes Autos $35 \%$ " " " rotes Auto $R$

12 nicht rotes Autos: $65 \%$ " " " nicht " " $\bar{R}$

13 wir haben zwei möglichkeiten: einmal der Doppelte baumdiagramm

14 und zweite der vier feldertafel

15 Ich würde die beiden möglichkeiten vorzeichnen, dass du die lernst.

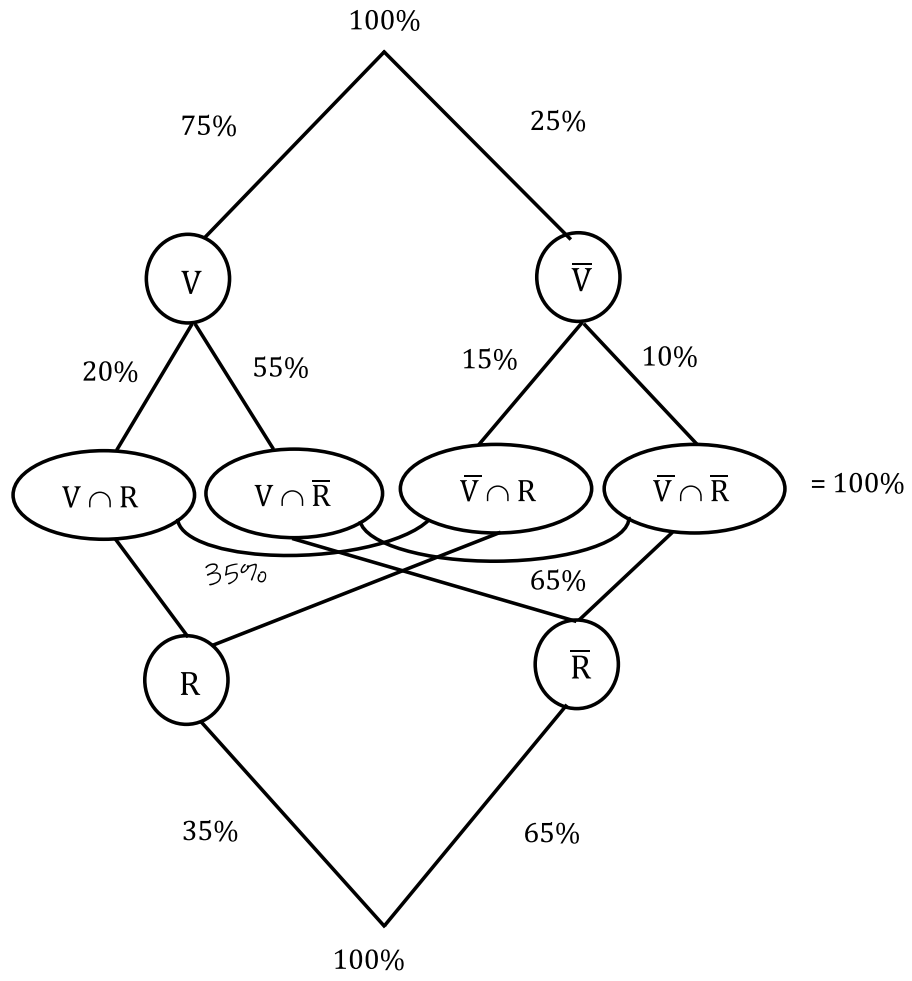

\begin{tabular}{|c|c|c|c|}
\hline & $\mathrm{V}$ & $\overline{\mathrm{V}}$ & $\mathrm{S}$ \\
\hline $\mathrm{R}$ & $20 \%$ & $15 \%$ & $35 \%$ \\
\hline$\overline{\mathrm{R}}$ & $55 \%$ & $10 \%$ & $65 \%$ \\
\hline $\mathrm{S}$ & $75 \%$ & $25 \%$ & $100 \%$ \\
\hline
\end{tabular}

- Abb. 2.14 Schülerdokument zum Thema ,Wahrscheinlichkeit“ 
Ansätze zur Genredidaktik, bei der die Strukturen, Funktionen und sprachlichen Mittel einer Fachtextsorte systematisch erarbeitet werden. ${ }^{11}$

Im vorliegenden Beispiel richtet der Schüler seinen Brief an den fiktiven Mitschüler Ömer, um ihm das aktuelle stochastische Thema zu erklären. Da im Unterricht zu diesem Zeitpunkt die bedingten Wahrscheinlichkeiten behandelt wurden, wäre es naheliegend gewesen, speziell darauf einzugehen, jedoch formuliert der Schüler offener: „Ich habe dir das Brief geschrieben um dir zuerklären wie geht mit dem Wahrscheinlichkeit" (02-03). Er wählt sein Beispiel so, dass er als Merkmal 1 die Autofarbe (rot bzw. nicht rot) und als Merkmal 2 das Alter von Schülern (volljährig bzw. nicht volljährig) nennt: „Wir haben autos die Rot sind und die nicht rot sind und wir haben schüler die volljährig und die nicht volljärig sind.“ (Z. 04-06). ${ }^{12}$ Dazu formuliert er (für seinen Adressaten Ömer) beispielhaft zwei Aufgabenstellungen (Z. 06-08):

a) wie Wahrscheinlich dass der volljähriger Schüler ein rotes auto besitzt und

b) die nicht volljähriger Schüler die Kein rotes auto besitzen.

Damit die Aufgaben gelöst werden können, stellt er außerdem die relativen Häufigkeiten für die volljährigen bzw. nicht volljährigen Schüler sowie für die roten und nicht-roten Autos zur Verfügung (Z. 09-12). Er verwendet dabei geeignete mathematische Symbole für „Ereignis“ und „Gegenereignis“ (V und $\bar{V}$; R und $\bar{R}$ ), ohne allerdings diese beiden zentralen Fachbegriffe auch zu benennen. Zudem wählt er zwei geeignete graphische Darstellungen, nämlich das doppelte Baumdiagramm und die Vierfeldertafel (siehe Z. 13-14 sowie die entsprechenden Darstellungen), in die er die Zahlen aus seinem Beispiel überführt. Dies gelingt ihm - allerdings mit einer mathematisch relevanten Ausnahme: Im Baumdiagramm trägt er auf die Pfade 2. Grades fälschlicherweise die Schnittwahrscheinlichkeiten $(20 \%, 55 \%, 15 \%$ und $10 \%)$ anstelle der bedingten Wahrscheinlichkeiten ab (richtig wäre also: $\frac{20}{75}, \frac{55}{75}, \frac{15}{25}, \frac{10}{25}$ ). Auch die vom Schüler selbst formulierten Aufgaben lassen darauf schließen, dass er den Unterschied zwischen Schnittwahrscheinlichkeit und bedingter Wahrscheinlichkeit noch nicht sicher beherrscht. Aufgabe a) scheint auf die bedingte Wahrscheinlichkeit abzuzielen - also $\mathrm{P}_{\mathrm{V}}(\mathrm{R})$ : Die Wahrscheinlichkeit, dass das Auto rot ist, wenn man weiß, dass der Besitzer volljährig ist. Im Unterschied dazu legt seine Aufgabe b) nahe, dass nach der Schnittwahrscheinlichkeit gefragt wird also $\mathrm{P}(\overline{\mathrm{V}} \cap \overline{\mathrm{R}})$ : Die Wahrscheinlichkeit, dass die Person zugleich nicht volljährig ist und ein nicht-rotes Auto besitzt. In den vom Schüler am Ende des Dokuments zur Verfügung gestellten Lösungen hingegen sind für beide Aufgaben die Schnittwahrscheinlichkeiten angegeben $(, a)=20 \% ; b)=10 \%$ "). Insgesamt ist also davon auszugehen, dass das Thema noch nicht ausreichend gefestigt ist.

Die Analyse des Zusammenhangs von sprachlichen und fachlichen Aspekten verdeutlicht eine Vielzahl von Phänomenen, von denen hier nur einige wenige diskutiert werden. Aus struktureller Perspektive ist zunächst festzuhalten, dass der Schüler sein Dokument inhaltlich plausibel strukturiert. So wird zunächst der allgemeine Rahmen und das Ziel benannt (Einführungssatz), dann eine Einführung in den Kontext gegeben (Explizierung der Merkmale), um im weiteren Verlauf eine Mathematisierung vorzunehmen, indem der Schüler die relativen Häufigkeiten benennt und die Merkmale sprachlich durch geeignete Symbole kennzeichnet. Gerade in diesem Schritt findet nicht nur ein wichtiger mathematischer Strukturierungsprozess statt, sondern auch ein sprachlicher. So ist an die-

11 Vgl. Frank und Gürsoy (2014), Jahn (2020).

12 Aus realitätsbezogener Sicht lässt sich an diesem Beispiel natürlich u. a. diskutieren, wie plausibel es ist, dass nicht-volljährige Personen überhaupt ein Auto besitzen. 
ser Stelle etwa der Unterschied zwischen relativen Häufigkeiten (die eine entsprechende Verteilung angeben) und den (gesuchten) Wahrscheinlichkeiten (in dem Fall z. B. dafür, dass ein volljähriger Schüler ein rotes Auto besitzt) von Bedeutung. Für diese inhaltlich wichtige Unterscheidung zwischen realen Verteilungen und prognostischen Aussagen braucht es natürlich auch entsprechende sprachliche Mittel - fachliche Vorstellungen und sprachliche Mittel sind hier also unmittelbar miteinander verknüpft. So gibt der Schüler etwa „Volljährige 75\%“ (Z. 09) als relative Häufigkeit für die Verteilung an und bestimmt am Ende des Dokuments die entsprechenden Wahrscheinlichkeiten. Hinsichtlich der unterrichtspraktischen Umsetzung wäre in diesem Zusammenhang zu diskutieren, inwiefern die Berechnung der Wahrscheinlichkeiten noch genauer expliziert werden sollte. Ein geeigneter Auftrag für Studierende besteht in diesem Zusammenhang darin, entsprechende Sprachmittel für die Thematisierung von relativen Häufigkeiten, Schnittwahrscheinlichkeiten und bedingten Wahrscheinlichkeiten zu finden und diese hinsichtlich der fachlichen Passung kritisch zu reflektieren. Weiterhin lässt sich anhand des vorliegenden Beispiels der Aspekt der Arbeit mit Darstellungen bzw. der Darstellungsvernetzung thematisieren. Gerade mathematische Objekte - aus mathematikphilosophischer Perspektive verstanden als genuin theoretische Objekte - sind ausschließlich über Darstellungen (z. B. in symbolischer Form mit Hilfe von Termen oder über entsprechende graphische Repräsentationen) erfahrbar. So betont Duval:

॥ Mathematical objects, in contrast to phenomena of astronomy, physics, chemistry, biology, etc., are never accessible by perception or by instruments (microscopes, telescopes, measurement apparatus). The only way to have access to them and deal with them is using signs and semiotic representations (Duval 2006, S. 107).

Für das inhaltliche Denken im Fach Mathematik ist die Vernetzung unterschiedlicher Darstellungsformen zentral (Duval 2006; Prediger und Wessel 2013 u. v. m.). So betont etwa Duval (2006) weiter:

I) Changing representation register is the threshold of mathematical comprehension for learners at each stage of the curriculum. It depends on coordination of several representation registers and it is only in mathematics that such a register coordination is strongly needed (Duval 2006, S. 122).

Im vorliegenden Kontext sind die vielfältigen Forschungsbefunde zur Rolle der Darstellungsvernetzung für die Sprachbildung im Fach Mathematik daher von besonderer Bedeutung (vgl. z. B. Zindel 2019; Wessel 2015; Moschkovich et al. 1993; Romberg et al. 1993; Moschkovich 1998).

Für die Diskussion der Zusammenhänge zwischen fachlichem und sprachlichem Lernen lohnt es sich, im Seminar die sprachlichen Mittel, die man für die Arbeit mit den unterschiedlichen Darstellungsformen benötigt, zu spezifizieren. Diese Analyse kann in Praxisphasen des Studiums natürlich auch mit den Schülerinnen und Schülern vorgenommen werden und sie erbringt nicht nur sprachliche, sondern auch elementare fachliche Einsichten. So sind etwa die Pfadwahrscheinlichkeiten ein wesentliches Merkmal des (doppelten) Baumdiagramms. Im Kontext bedingter Wahrscheinlichkeiten spielen sie insofern eine zentrale Rolle, als bei zweistufigen Zufallsversuchen die Pfadwahrscheinlichkeiten der zweiten Stufe den bedingten Wahrscheinlichkeiten entsprechen. In diesem Zusammenhang sollte die Beziehung zur symbolischen Darstellung thematisiert werden, die im Satz von Bayes zum Ausdruck kommt. Mit Hilfe des Baumdiagramms und der 
damit verbundenen Sprachmittel bekommen Lernende mithin fachliche und sprachliche Gelegenheiten der sinnstiftenden Erarbeitung der entsprechenden Inhalte.

Für Studierende macht das obige Schülerbeispiel aber auch deutlich, in welcher Weise Schülerinnen und Schüler mathematische Gegenstände in eigenen Worten schriftlich festhalten (können). Viele Studierende sind häufig zunächst überrascht, dass Schülerinnen und Schüler (insbesondere solche, die die deutsche Sprache erst seit kurzem erlernen) solche beeindruckenden Texte formulieren können (und, wie Erfahrungen aus der Zusammenarbeit mit Schulen zeigen, dies oft auch durchaus bereitwillig tun).

Insgesamt ist die Arbeit mit empirischen Daten aus dem Unterricht für viele Studierende herausfordernd, weil sie sowohl sprachanalytische Kenntnisse als auch ein sehr genaues Verständnis der mathematischen Gegenstände erfordert. Denn nur vor dem Hintergrund der differenzierten fachlichen Betrachtung lässt sich einschätzen, welche Sprachmittel etwa für die Unterscheidung von (realen) Verteilungen und den entsprechenden relativen Häufigkeiten sowie prognostischen Aussagen mit den entsprechenden Wahrscheinlichkeiten geeignet bzw. weniger geeignet erscheinen. Der Kontext der Wahrscheinlichkeitsrechnung ist in diesem Zusammenhang durchaus bewusst gewählt: Gerade weil die mathematischen Aktivitäten in der Regel in alltagsnahen Kontexten verortet sind und zudem viele Fachbegriffe mit der Alltagssprache interferieren, ist die genaue sprachliche (und damit auch fachliche!) Reflexion hier besonders ergiebig.

Nicht selten entsteht auf der Grundlage der Arbeit mit solchen Schülerdokumenten der Wunsch, in Praxisphasen ähnliche Beispiele zu sammeln und wissenschaftlich genauer zu untersuchen. Dies kann in der Regel recht niedrigschwellig geschehen und bietet für entsprechende Reflexionsphasen (z. B. in Begleitveranstaltungen zum Praxissemester) vielfältige Anlässe für analytische Betrachtungen.

\section{- Impulse und Hilfestellungen für Studierende bei der Arbeit mit Schülerprodukten}

Für die Arbeit mit Schülerprodukten in der Hochschullehre gelten ganz ähnliche Prinzipien wie für den (Mathematik-)Unterricht: Gerade bei Fragen von Studierenden in Arbeits- und Analysephasen ist es wichtig, Rückmeldungen bewusst vorzunehmen. Zech (1998) nimmt in diesem Zusammenhang eine Kategorisierung von Rückmeldungen vor und unterscheidet zwischen eher prozess- und eher inhaltsbezogenen Rückmeldungen.

Im Rahmen einer Analyse von Schülerprodukten durch die Studierenden versteht man z. B. unter einer eher prozessorientierten Rückmeldung bzw. Hilfestellung folgende Impulse:

- Formulieren Sie die theoretischen Konstrukte für die Analyse der Schülerbeispiele noch einmal in eigenen Worten und wenden Sie diese dann auf die empirischen Daten an.

- Wählen Sie zwei (drei, vier) theoretische Konstrukte bzw. Kategorien aus und erfinden Sie (ggf. etwas überzeichnete) Schülerbeispiele für den vorliegenden Gegenstandsbereich. Nehmen Sie nun eine Zuordnung der realen Daten vor.

Eher inhaltsorientierte Rückmeldungen sind hingegen etwa:

- Die gefundenen Kategorien sind richtig/falsch zugeordnet.

- Die bisherigen Arbeitsergebnisse entsprechen eher einem anderen theoretischen $\mathrm{Zu}$ gang.

Im Rahmen der Hochschullehre ist es durchaus angebracht, Hilfen und Rückmeldungen zunächst auf Prozessebene zu geben, bevor man eine inhaltliche Einschätzung vornimmt (dazu auch Zech 1998), um den Studierenden die Gelegenheit zu geben, sich die Inhalte selbsttätig anzueignen. 


\subsection{Sprache und Fach erkunden durch forschendes Lernen}

॥ Ich empfinde den universitären Teil des Praxissemesters nicht als zu wissenschaftlich. Ich denke, es ist eine Chance für die Studierenden, so zu arbeiten. Das schließt nicht aus, dass ich im Berufsalltag noch weiter forsche, ich habe hier ja die Methoden kennengelernt (Student, Praxissemester 2018).

Ein zentrales Ziel des Lehramtsstudiums ist es, dass die Studierenden sinnvolle und nützliche Verbindungen zwischen universitärer Ausbildung und schulischer Praxis herstellen können, die zu ihrer Professionalisierung beitragen. Dabei sollen die Studierenden erfahren, dass ihnen theoretische Konzepte aus den verschiedenen Disziplinen helfen, Sichtweisen oder Probleme von Lernenden nachzuvollziehen und daraus Handlungsmöglichkeiten für ihren Unterricht abzuleiten. Umgekehrt kann durch in der Schulpraxis aufkommende eigene Fragen neues Interesse an wissenschaftlichen Erklärungen entstehen (Weyland 2019, S. 43). Das forschende Lernen, wie es z. B. in den Studienprojekten im Praxissemester vorgesehen ist, bietet dafür einen guten Rahmen: Die Studierenden entwickeln eine theoretisch begründete, praxisrelevante und für sie interessante Fragestellung - im vorliegenden Fall für das Fach Mathematik und mit einem Schwerpunkt auf Sprachbildung - und führen dazu ein kleineres empirisches Projekt an der Schule durch.

Für viele Studierende stellt das forschende Lernen erfahrungsgemäß eine große Herausforderung dar. Wir halten den Ansatz für sehr sinnvoll, wenn es gelingt, bei den Studierenden eine phänomenbezogene, prozessorientierte und offene Haltung anzubahnen. Idealerweise unterstützt das forschende Lernen die Studierenden in Bezug auf alle in den Leitideen ( Abschn. 1.2) formulierten Fähigkeiten: Durch die intensive forschende Auseinandersetzung mit Sprachbildung im Fach Mathematik, die i. d. R. die Konzeption von Aufgaben sowie die kriteriengeleitete Auswertung der erhobenen Lernendendaten umfasst, werden alle zentralen Aspekte der Professionalisierung - Sensibilisierung für Sprache im Fach Mathematik (L1), Aufgabengestaltung (L2), unterstützende Maßnahmen (L3) und fachlich-sprachliches Diagnostizieren (L4) - angesprochen.

Hinsichtlich der hochschuldidaktischen Design-Prinzipien wird in diesem Abschnitt die Integration von Sprache und Fach insbesondere auf der Ebene des Forschenden Lernens (DP4) thematisiert. Neben Hinweisen zur Planung und Durchführung von Studienprojekten werden auch hochschuldidaktische und methodische Aspekte zur Präzisierung von Forschungsdesigns diskutiert. Viele Aspekte lassen sich auch auf andere (interdisziplinäre) Veranstaltungen im Kontext von Praxisphasen übertragen.

\subsubsection{Forschendes Lernen im Praxissemester}

Mit der Reform der Lehramtsausbildung in Nordrhein-Westfalen (2009) wurde ein fünfmonatiges Praxissemester im 2. Semester des Masterstudiums verankert. Die Studierenden hospitieren und unterrichten in dieser Zeit an einer Schule. Sie führen zugleich theoriegeleitete Erkundungen durch, d. h. sie untersuchen einen kleinen Ausschnitt des Handlungsfelds Schule und gewinnen daraus Erkenntnisse für ihre zukünftige Tätigkeit als Lehrerinnen und Lehrer. Ziel des Studienprojekts ist es, dass die Studierenden beobachtete Phänomene unabhängig von subjektiven Eindrücken untersuchen, indem sie Theorie und Praxis aufeinander beziehen. Das Studienprojekt soll ,im Sinne eines forschenden Habitus“ (MSW NRW 2016, S. 5) erfolgen und die explorierende, 
kritisch-reflexive Grundhaltung der Studierenden fördern. Die fachliche Betreuung der Studienprojekte erfolgt in den fachdidaktischen bzw. bildungswissenschaftlichen Begleitseminaren an der Universität.

Die erste Evaluation in Nordrhein-Westfalen (2016) hat ergeben, dass das Praxissemester grundsätzlich insgesamt sehr positiv bewertet wird, weil es einen ,intensiven Einblick in den Arbeitsplatz Schule“ (MSW NRW 2016, S. 7) ermöglicht. Allerdings halten $78 \%$ der befragten Studierenden die Anforderungen der Studienprojekte für zu hoch und immerhin ein Drittel der Studierenden schätzt die universitäre Unterstützung im Praxissemester u. a. wegen unzureichender Praxisrelevanz als kritisch ein (ebd.). Es stellt sich also die Frage, wie die Begleitseminare zum Praxissemester so gestaltet werden können, dass sie den Studierenden in dieser zentralen Studienphase bestmögliche Unterstützung bieten und zugleich die Bedeutung einer wissenschaftlichen Herangehensweise für die eigene Professionalisierung verdeutlichen; dies gilt umso mehr für interdisziplinäre Seminare, die aufgrund der notwendigen Berücksichtigung zweier Disziplinen noch einmal besondere Anforderungen stellen. Für die Gestaltung von Lehrveranstaltungen zum forschenden Lernen in Praxisphasen sind u. a. folgende Punkte von Bedeutung:

- vorbereitende Übungen zum forschenden Lernen; dazu gehört insbesondere die Arbeit mit empirischen Daten (z. B. Audio-/Videoaufnahmen, Transkripte, schriftliche Schülerdokumente);

- vielfältige Beratungs- und Diskussionsanlässe zur kontinuierlichen Weiterentwicklung des Studienprojekts;

- theoretische Grundlagen, die helfen, schulische Praxis zu reflektieren, zu erklären, zu systematisieren und zu verbessern;

- Anlässe zur Reflexion, bei denen die Studierenden ihre Kompetenzentwicklung und Einflüsse, die dabei eine Rolle gespielt haben, klar erkennen können; idealerweise sollten die Studierenden in der Lage sein, die gewonnenen Erkenntnisse in ihrer weiteren Laufbahn weiter zu nutzen.

Dass die Anforderungen der Studienprojekte als zu hoch wahrgenommen werden, ist nicht überraschend; die meisten Studierenden haben nur wenig Erfahrung mit der eigenständigen Planung und Durchführung einer empirischen Studie. Sie benötigen daher gezielte Unterstützung in allen Phasen des forschenden Lernens:

- bei der Entwicklung einer geeigneten Forschungsfrage,

- beim Finden passender Forschungsmethoden,

- bei der Gestaltung der Datenerhebung,

- bei der Analyse und Diskussion der erhobenen Daten.

Die Studienprojekte sollten so angelegt sein, dass die Studierenden daraus möglichst viele Erkenntnisse für sich ziehen. Das geht i.d. R. über die reine Beobachtung hinaus und erfordert häufig die Einbeziehung mündlicher und/oder schriftlicher Lernendendaten. Gerade für solche deskriptiven empirischen Projekte eignet sich der Ansatz des forschenden Lernens. Die Daten müssen in diesem Zusammenhang nicht unbedingt selbst erhoben werden: Es können beispielsweise auch Schülerprodukte wie Lerntagebücher oder Klausurbearbeitungen untersucht werden, die ohnehin im Unterrichtskontext entstehen (Caspari 2016). Die Identifikation mit dem Projekt und das Lernpotential für die Studierenden ist aber unter Umständen höher, wenn sie ,ihre“ Daten selbst erheben, zumal dies im Fach Mathematik oftmals die eigenständige Entwicklung und Erprobung von Aufgabenstellungen bedeutet. 


\subsubsection{Verzahnung der Lernorte Schule und Universität}

- Abb. 2.15 skizziert den zeitlichen Ablauf forschenden Lernens am Beispiel des Studienprojekts im Praxissemester; dabei wird die enge Verzahnung der Lernorte Schule und Universität deutlich. Das Begleitseminar zum Praxissemester findet in drei Blockveranstaltungen statt. Die in der Abbildung genannten Aktivitäten Forschungsfragen generieren, Speed-Dating und Diskussion in Arbeitsgruppen werden in Abschn. 2.5.4 am Beispiel des Schwerpunkts „Sprachbildung im Fach Mathematik“ beschrieben.

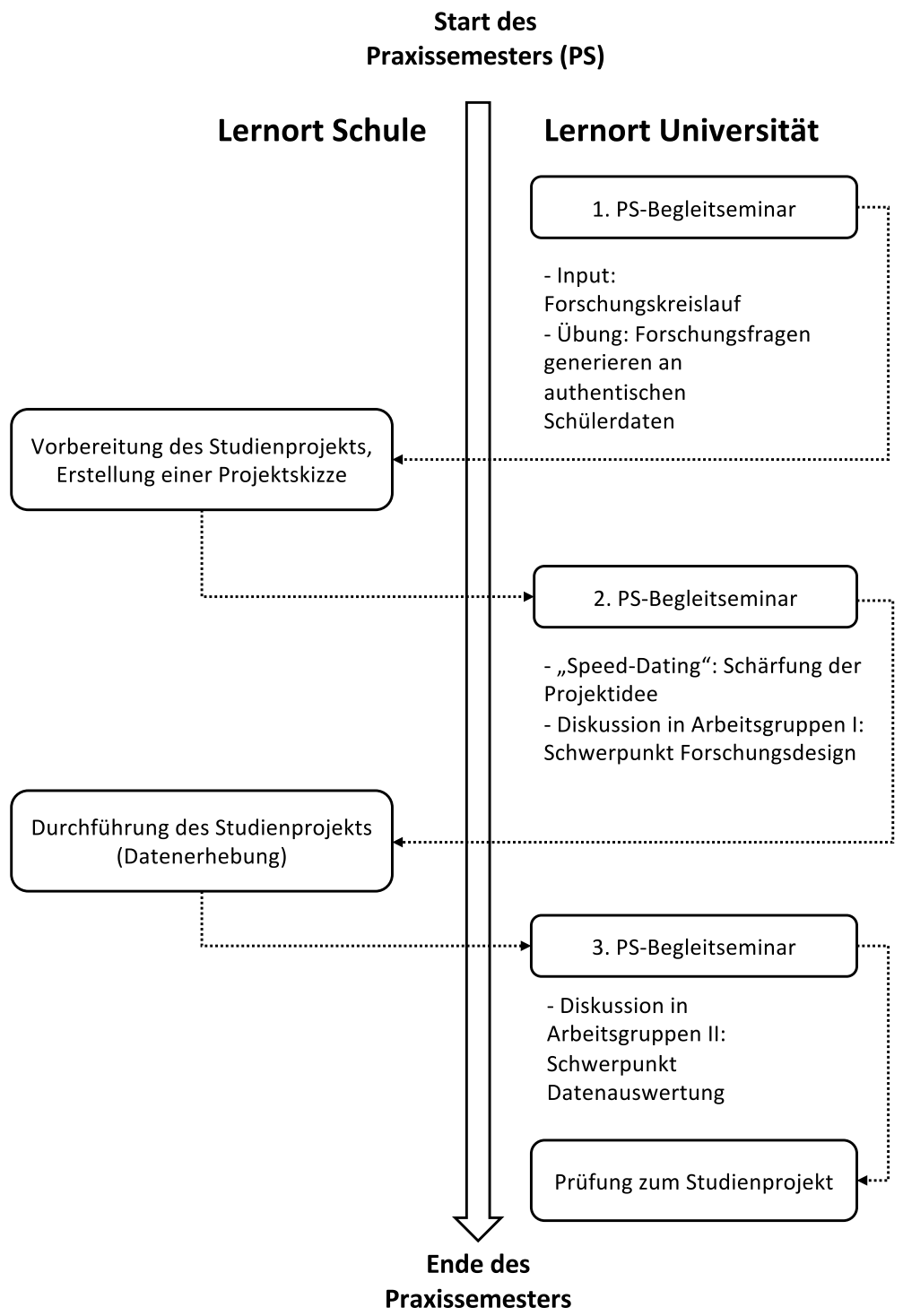

- Abb. 2.15 Studienprojekt: Verzahnung der Lernorte Schule und Universität 
Das erste Begleitseminar findet statt, kurz nachdem die Studierenden ihr Praxissemester in den Schulen gestartet haben. Zu diesem Zeitpunkt ist meistens noch nicht klar, in welchen Klassen oder mit welchen Schüler*innen ein Studienprojekt sinnvollerweise durchgeführt werden kann. Daher werden im ersten Begleitseminar zunächst allgemeine Fragen zu Elementen und Ablauf eines empirischen Projekts besprochen. Einen besonderen Schwerpunkt bildet das Entwickeln von Forschungsfragen (dazu im Einzelnen auch Abschn. 2.6). Die Studierenden haben dann etwa sechs Wochen Zeit, um ihr Studienprojekt an der Schule vorzubereiten und eine vorläufige Projektskizze zu erstellen. Im zweiten Begleitseminar stellen die Studierenden ihre Projektskizzen vor und entwickeln sie gemeinsam weiter. Alle Studierenden sollten spätestens am Ende des zweiten Begleitseminars eine klare Vorstellung bezüglich ihrer Fragestellung und ihres Forschungsdesigns haben. Die Datenerhebung findet zwischen dem zweiten und dritten Begleitseminar an den Schulen statt. Der Fokus des dritten Seminars liegt entsprechend auf der Auswertung der erhobenen Daten. Den Abschluss des Praxissemesters am Lernort Universität bildet die mündliche Prüfung, in der das Studienprojekt vorgestellt und diskutiert wird.

Die Aktivitäten an der Schule und an der Universität sind, wie - Abb. 2.15 verdeutlicht, zeitlich und inhaltlich aufeinander abgestimmt. Auf diese Weise kann eine enge Verknüpfung von Praxiserfahrung und forschendem Lernen hergestellt werden.

\subsubsection{Stiftung von Kohärenz beim forschenden Lernen}

Die meisten Studierenden verfügen, auch wenn sie vielleicht noch nicht eigenständig geforscht haben, über Grundkenntnisse darüber, wie eine empirische Studie durchgeführt wird. So wissen sie, dass eine geeignete Forschungsfrage wichtig ist, sie kennen unterschiedliche Forschungsmethoden, und sie wissen, dass erhobene Daten unter Rückbezug auf theoretische Konzepte interpretiert werden müssen. Auch die an Schulen geltenden Datenschutzrichtlinien sind i. d. R. bereits bekannt.

Anhand eines Forschungskreislaufs (- Abb. 2.16) kann das Wissen der Studierenden gesammelt, ergänzt und systematisiert werden.

Die einzelnen Elemente des Forschungskreislaufs werden im Folgenden genauer beschrieben und in Beziehung zueinander gesetzt.

- Abb. 2.16 Forschungskreislauf

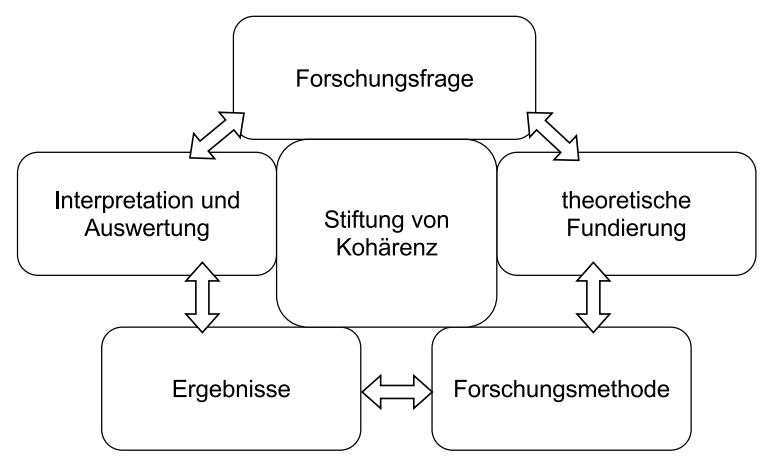


Den Ausgangspunkt bildet die Ausbildung eines Forschungsinteresses und damit verbunden die Formulierung einer vorläufigen Forschungsfrage. Sie ist notwendig, damit wichtige Entscheidungen z. B. hinsichtlich Probandenauswahl und Forschungsmethoden getroffen werden können. So betont etwa Riemer (2014), es sei

॥ ein [...] Trugschluss, dieser grundlegenden forschungsmethodologischen Entscheidung nicht die nötige Bedeutung zuzuweisen - auch bzw. gerade in qualitativer Forschung ist die Forschungsfrage auszudifferenzieren, will man einen langen Aufenthalt im WO-BINICH-NEBEL vermeiden, der zu einem viel späteren Zeitpunkt im Forschungsprozess droht, wenn die Datenerhebungen und -analysen unter zu vage formulierten Fragestellungen vorgenommen wurden (Riemer 2014, S. 23).

Das schließt aber natürlich nicht aus, dass sich die vorläufige Forschungsfrage im Verlauf des Studienprojekts aufgrund der Datenlage oder mit steigendem Wissensstand weiterentwickelt (also z. B. der Fokus auf einen ausgewählten Teilaspekt der ursprünglichen Fragestellung gerichtet wird). Die Studierenden sind im Rahmen der hier beschriebenen Lehrveranstaltungen in der Wahl ihrer Forschungsfrage weitgehend frei, sie müssen aber den Aspekt der Sprachbildung mitberücksichtigen.

Jedes Studienprojekt muss theoretisch fundiert sein. Die theoretischen Grundlagen aus den Fachwissenschaften und -didaktiken helfen bei der Ausschärfung der Forschungsfrage, bei der Entwicklung des Forschungsdesigns und bei der Interpretation der Daten. So werden aus dem Studium bereits bekannte ebenso wie neue theoretische Ansätze durch das forschende Lernen in direkten Zusammenhang mit dem Handlungsfeld Schule gebracht. In unserem Kontext sind zum Beispiel das didaktische Prinzip der Darstellungsvernetzung, Unterschiede zwischen alltäglichem und fachlichem Begriffsverständnis, mathematische Grund- und Fehlvorstellungen sowie die sprachbewusste Aufgabenvariation relevante Themen. Das Studienprojekt eröffnet die Möglichkeit, diese auf theoretischer Ebene bekannten Zugänge zu Unterricht und Lernen in einem konkreten Handlungskontext (wenn auch oft exemplarisch) umzusetzen, zu erproben und kritisch zu überprüfen. Im besten Fall wird damit das „Eintauchen“ in Wissenschaft gefördert; damit könne

॥ der Nutzen wissenschaftlichen Wissens als Theorie- und Begründungswissen für professionelles pädagogisches Handeln verdeutlicht und der im Forschungszusammenhang zur Lehrerbildung durchaus anzutreffenden Aussage einer Theoriefeindlichkeit zielführend begegnet werden (...) (Weyland 2019, S. 43, Kursivierung im Original).

Mit der hier ausgearbeiteten Konzeption für eine sprachbewusste Hochschullehre werden Erfahrungen geschildert, die sicher sehr stark mit den aktuellen Forschungspraxen des Autorenteams verknüpft sind. Vor diesem Hintergrund wurden im Rahmen von Studienprojekten gute Erfahrungen u. a. mit folgenden Forschungsmethoden gemacht:

- strukturierte Beobachtung von Unterricht,

- systematische Erhebung und Analyse von schriftlichen Schülerdokumenten (Aufgaben- und Testbearbeitungen, Lerntagebücher, Poster usw.),

- systematische Erhebung und Analyse von (transkribierten) Audio-/Videoaufnahmen,

- mündliche und schriftliche Befragungen (z. B. diagnostisches Interview). 
Dabei werden mit den Studierenden die jeweiligen Vor- und Nachteile, Anwendungsszenarien sowie mögliche Kombinationen von Forschungsmethoden diskutiert. Beobachtungen und Befragungen unterstützen wir, sofern sie im Zusammenhang mit der Analyse von mündlichen oder schriftlichen Schülerprodukten stehen (z. B. Befragung einzelner Schüler*innen zu Aufgabenlösungsprozessen).

Die Datenerhebung erbringt Ergebnisse, die mit Blick auf die Fragestellung, die theoretischen Grundlagen und die Methode interpretiert und ausgewertet werden müssen. Dabei können z. B. folgende Fragen auftreten: Kann ich mit meinen Ergebnissen die Forschungsfrage sinnvoll beantworten? Welche theoretischen Grundlagen helfen mir bei der Interpretation? Oder, bei der Anwendung von mehreren Methoden: Welche Methode gibt Aufschluss worüber?

Bei der Ergebnissichtung und Datenauswertung sind zwei Tendenzen festzustellen:

1) Die Studierenden haben zu viele Daten erhoben. Da die Auswertung aller Daten den Rahmen des Studienprojekts sprengen würde, müssen sie eine sinnvolle Auswahl treffen.

2) Die Studierenden sind entmutigt, weil die Daten auf den ersten Blick wenig ergiebig erscheinen; sie brauchen bei der Auswertung besondere Unterstützung (z. B. durch die gemeinsame Diskussion eines auswählten Datenbeispiels aus verschiedenen Perspektiven).

Ein wichtiger Hinweis zu Beginn des Studienprojekts und vor der Datenerhebung ist daher, dass es nicht um die Erhebung großer Datenmengen oder um besonders ,gelungene" Daten geht, sondern dass vielmehr die detaillierte, theoretisch fundierte Interpretation weniger Daten mit dem Ziel eines persönlichen Erkenntnisgewinns im Vordergrund steht.

\subsubsection{Begleitung der studentischen Projekte im Rahmen der Hochschullehre}

Im Folgenden werden ausgewählte Formate für die Gestaltung von Lehrveranstaltungen vorgestellt, die Studierenden bei der Durchführung eines kleinen empirischen Projekts, also z. B. eines Studienprojekts im Praxissemester, helfen können. Die Übungen zeigen, dass forschendes Lernen durch die Unterstützung von Mitstudierenden und Lehrenden durchaus zu bewältigen ist. Durch den intensiven Austausch im Seminar erlangen die Studierenden innerhalb kurzer Zeit Einblicke in eine Vielfalt an mathematischen Lernbereichen, Möglichkeiten der Sprachbildung im Mathematikunterricht und Methoden der Datenerhebung und -auswertung. Fach- und sprachdidaktische Ansätze werden aufgegriffen, miteinander vernetzt und auf ihren Nutzen im jeweiligen schulpraktischen Projektkontext hin überprüft, so dass eine Vermittlung zwischen den manchmal als getrennt wahrgenommenen universitären und schulpraktischen Ausbildungsanteilen hergestellt werden kann.

\subsubsection{Finden von Forschungsfragen}

Die folgende Übung eignet sich, um das Finden und Formulieren von theoretisch fundierten Forschungsfragen zu üben. Die Grundlage bilden empirische Daten, wie sie auch im Rahmen eines eigenen (Studien-)Projekts erhoben werden könnten. Transkribierte 
- Abb. 2.17 Schülerdokument zur Aufgabe „Schulfest“ (Auszug) d) Wir haben die Werte dem Text entnommen und sie dann in das Baumdiagramm übertragen.

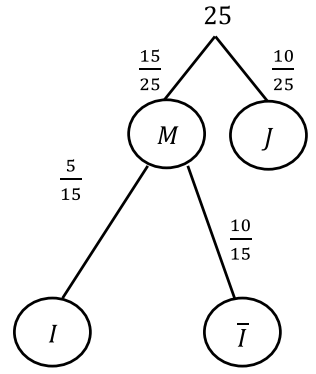

Audio- oder Videoaufnahmen sind dafür gut geeignet, weil sie einen direkten Einblick in Lernprozesse ermöglichen. Es können aber natürlich auch andere Dokumente (z. B. Lerntagebücher, Wissensspeicher, Konstruktionsbeschreibungen, schriftliche Aufgabenlösungen, Lehrmaterial usw.) genutzt werden.

Wir verwenden als Material die Audioaufnahme zweier Zehntklässlerinnen, die sie bei der gemeinsamen Bearbeitung einer Stochastikaufgabe mit der Diktierfunktion ihres Handys selbst erstellt haben. Die Studierenden erhalten:

- die Textaufgabe zum Thema Schulfest (vgl. Abschn. 2.3), die die Schülerinnen bearbeitet haben,

- einen Ausschnitt der schriftlichen Notizen, die die beiden Schülerinnen im Verlauf der Aufgabenbearbeitung erstellen (- Abb. 2.17),

- einen Ausschnitt aus der Audioaufnahme als Transkript.

Im abgedruckten Transkriptausschnitt sprechen die Schülerinnen über den Aufgabenteil d).

\section{Aufgabe "Schulfest"}

Aufgabe 1) Für das Schulfest werden noch Musiker gesucht. Die Klasse 9a hat unter den 25 Schülerinnen und Schülern eine Umfrage gemacht. Diese ergab, dass jedes dritte Mädchen ein Instrument spielt, während es bei den Jungen 2 von 10 sind.

a) Erstelle ein Baumdiagramm.

b) Stelle die Daten in einer Vierfeldertafel dar.

c) Bilde die Situation in einem Einheitsquadrat ab.

d) In der Klasse werde zufällig eine Person ausgewählt. Begründe für jede Darstellungsform, wie sich die Wahrscheinlichkeit für das Ereignis Mädchen $\cap$ Instrument bestimmen lässt.

(Guckelsberger und Schacht 2018) 
(s01) S1: Also d) ist dann ((liest vor)): „In deiner Klasse wer/ wer/ In der Klasse werde zufällig eine Person ausgewählt. Begründe für jede Darstellungsform, wie sich die Wahrscheinlichkeit für das Ereignis Mädchen und Instrument bestimmen lässt.“

(s02) Okay.

(s03) Also müssen wir jetzt jedes Ereignis machen oder wie?

(s04) Aber eigentlich suchen die doch nur Mädchen und Instrument. ((2s))

(s05) ((liest vor)) „In der Klasse werde zufällig eine Person ausgewählt.“

(s06) Kann man das nicht ,,in der Klasse wird einfach eine Person ausgewählt“ schreiben?

(s07) ((liest vor ) ),Begründe für jede Darstellungsform, wie sich die Wahrscheinlichkeit für das Ereignis“/

(s08) S2: Vielleicht sollen wir ein Baumdiagramm und 'ne Vierfeldertafel machen?

(s09) S1: Haben wir ja!

(s10) S2: Nein, zu der Aufgabe • nochmal, ((1s))

(s11) S1: Ach so, du meinst $\bullet$ dieses halbe Baumdiagramm.

(s12) Mit (unverständlich). Wo wir nur den Pfad machen, der uns interessiert.

(s13) Okay. $\bullet$ Okay.

(s14) Dann machen wir ein doppeltes oder ein ein/ einfaches?

(s15) S2: Einfaches.

(s16) S1: Einfaches, okay. Ähm, dann haben wir einmal Mädchen und Jungen, ne?

(s17) S2: Ja.

(s18) S1: Also „M“",J“.

(s19) Wieder/ wir gehen wieder von fünfundzwanzig aus..

(s20) Das sind dann/ Mädchen hatten wir gesagt/ wie viele?

(s21) Fünfzehn. Fünfundzwan/ äh, fünfzehn Fünfundzwanzigstel

(s22) S2: Zehn Fünfundzwanzigstel Jungs.

(s23) S1: Mhm, und dann lassen wir das mit den Jungs sein und dann gucken wir uns nur noch die Mädchen an.

(s24) Also Mädchen Instrument und kein Instrument dann direkt/

(s25) also das, was wir eigentlich auch schon hatten, ne?

(s26) Instrument, kein Instrument.

(s27) Und jetzt interesse/ interessiert uns ja nur das mit dem Instrument.

(s28) Das sind dann

(s29) S2: Fünf Fünfzehntel.

(s30) S1: Genau, mit dem Instrument sind fünf Fünfzehntel und das andere sind dann zehn Fünfzehntel.

(s31) S2: Ja. Hm. (unverständlich)

(s32) S1: ((liest vor)) „Begründe für jede Darstellungsform“/

(s33) ach so, nein!

(s34) Wir sollen begründen.

(s35) Für diese Darstellungsformen die/

(s36) Hm, warte.

(s37) Ach so! Wir haben das falsch gemacht.

(s38) Ach so, wir hätten das einfach nur begründen sollen. 
(s39) S2: Nur begründen sollen.

(s40) S1: Ja. Oh, okay. Ähm, okay. ((1 s))

(s41) Wie ist die Wahrschein/ • W/ Wie sie sich bestimmen lässt? Ja, oder?

(s42) S2: Das kann man eigentlich ablesen.

(s43) S1: Ja.

(s44) Also schreiben wir $\bullet$ Mhm, „man kann das ablesen“.

(s45) Aber das ist ein bisschen• doof für ne Begründung, oder?

(s46) S2: Ähm, wir müssen jetzt die Zahl hier/ die Wahrscheinlichkeit • war/ ((1 s))

(s47) S1: Was ist/ was ist denn das für 'ne Begründung?

(s48) Ich meine, das ist doch klar, wenn wir das machen, dann können wir einen Pfad langgehen.

(s49) S2: Ja.

(s50) S1: Und den/ ich schreib einfach: „man“/ Ja, ,man sieht das doch“. ((3 s))

(s51) Ich weiß nicht, wie ich das sonst anders formulieren kann, hast du/

(s52) S2: Also wir müssen hier den ersten Pfad langgehen, also Mädchen und Instrument. Also das/

(s53) S1: Ja, also einfach den Pfad langgehen, ne?

(s54) S2: Ja, wir müssen die Zahl aufschreiben in der Antwort natürlich.

(s55) S1: Ach so

(s56) ähm also soll ich jetzt nicht schreiben „man sieht das“?

(s57) S2: Wir müssen/ Instrument.

(s58) Ich glaub', wir müssen • das malrechnen.

(s59) S1: Das wär' die Pfadmultiplikationsregel.

(s60) S2: Ja.

(s61) S1: Hier steht nur „begründe“ und nicht rechne. ((1 s))

(s62) Also ich meine, als Begründung kannst du auch ne Rechnung angeben.

(s63) ((liest vor)) „Begründe für jede Darstellungsform“/

(s64) S2: Ist das nicht Mädchen/ Heißt nicht das/ heißt das nicht „,kein Instrument“.

(s65) S1: Bestimmen/ aber hier ((liest vor)) ,,bestimmen lässt““.

(s66) S2: Oder ist das/

(s67) S1: Nein, oben „n“ [Anm.: S1 bezieht sich auf das mathematische Symbol $\cap$ in der Aufgabenstellung] heißt „und“.

(s68) Aber hier „,bestimmen lässt“, das heißt doch einfach, wie wir darauf gekommen sind.

(s69) S2: Ja. Dann musst du diese beiden Zahlen (unverständlich)

(s70) S1: Ich sag einfach: • „Man sieht das halt“".

(s71) S2: Ja, „das war erkennbar“. ((liest vor)) „Begründe“.

(s72) S1: Okay, warte.

(s73) Dann schreiben wir das ein bisschen schöner

(s74) Ähm, wenn man den Pfad entlang geht, erkennt man, wie viel/

(s75) Ach, ich schreib mal los.

(s76) S2: Schreib einfach die Zahlen. ((1 s))

(s77) S1: Ja, das ist auch keine gute Begründung.

(s78) S2: Fünfzehn/ ((1 s))

(s79) S1: Ja? 
(s80) S2: Die Wahrscheinlichkeit, dass ein Mäd/ das ist doch/ dass ein Mädchen ein Instrument spielt, ist doch die Frage, ne?

(s81) S1: Ja.

(s82) S2: Sind fünf Fünfzehntel.

(s83) Jetzt müssen wir sagen, wie wir darauf gekommen sind. ((2 s))

(s84) S1: Ähm, wir haben die Werte aus dem Text abgelesen.

(s85) Ah, das war ein guter Text.

(s86) ((schreibt), ,Wir $\bullet$ haben $\bullet$ die $\bullet$ Werte" dem Text/ aus dem Text entnommen.

(s87) Dem Text entnommen?

(s88) S2: Ja.

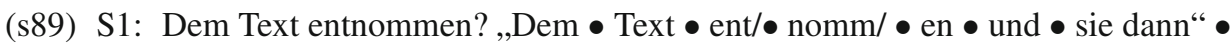

(s90) ich mach (unverständlich), oder?

(s91) S2: Also wir haben die fünfzehn durch drei geteilt, deswegen kam da ja fünf Mädchen raus und • die Gesamtzahl/ das ähm.

(s92) Nee, es gibt ja fünfzehn Mädchen, deswegen sind es fünf Fünfzehntel.

(s93) S1: Wir müssen ja nicht dieselbe Begründung haben.

(s94) Schreib einfach, was du geschrieben hast.

(s95) Ich hab' jetzt geschrieben: „Wir haben die Werte dem Text entnommen und sie dann in das Baumdiagramm übertragen."

(s96) Ich mein', was anderes haben wir ja nicht gemacht.

Dieses Material wird dann im Seminar aus möglichst unterschiedlichen Perspektiven „,befragt". Es ist zu beachten, dass bei dieser Übung also von bereits vorliegenden Daten ausgegangen wird, zu denen Fragen entwickelt werden, während bei der Planung des Studienprojekts der Prozess umgekehrt - von der Forschungsfrage zur Datenerhebung verläuft.

Wir halten die Übung für sehr nützlich, weil sie den Studierenden verdeutlicht, dass auch kleine Datenmengen reichhaltig sein können - die zugehörige Audioaufnahme hat eine Dauer von nur 4 min $53 \mathrm{~s}$ - und eine Bandbreite unterschiedlicher Fragerichtungen zulassen.

Der Arbeitsauftrag für die Studierenden lautet:

\section{Arbeitsauftrag an die Studierenden zur „Befragung" des empirischen Materials}

Lesen Sie den Transkriptausschnitt „Mädchen und Instrument“.

1) Setzen Sie sich zunächst inhaltlich mit dem Transkript auseinander. Inwiefern zeigt sich der Zusammenhang von fachlichem und sprachlichem Lernen?

2) Formulieren Sie anhand des Transkriptausschnitts und der schriftlichen Notizen der Schülerinnen fünf theoretisch fundierte Forschungsfragen.

\section{- Anmerkungen zum Arbeitsauftrag 1: Zusammenhang von sprachlichem und fachlichem Lernen}

Das Transkript bietet einen sehr guten (und in dieser Detailliertheit normalerweise nicht zugänglichen) Einblick, wie die beiden Schülerinnen bei der Aufgabenbearbeitung vor- 
gehen, welche Fragen auftreten und welche Annahmen aufgestellt und wieder verworfen werden. Die Studierenden haben so die Möglichkeit, kognitive und sprachliche Handlungen der Schülerinnen nachzuvollziehen und in ihrer Relevanz für das fachliche Lernen zu beurteilen. Dies ist sowohl für den unterrichtspraktischen als auch für den forschungsbezogenen Teil von Praxisphasen von Bedeutung. Zwei zentrale Punkte seien hier kurz benannt:

Im Transkriptausschnitt offenbart sich das Problem, dass den Schülerinnen der Unterschied zwischen bedingter Wahrscheinlichkeit und (der hier gesuchten) Schnittwahrscheinlichkeit nicht klar ist. Zwar interpretieren sie das mathematische Symbol für die Schnittmenge $(\cap)$ zunächst richtig (s01) (an späterer Stelle bezeichnen sie das Symbol hingegen fachlich nicht angemessen als „,“" (s67)). Unklarheit besteht jedoch hinsichtlich der jeweiligen Bezugsgrößen (alle Schüler vs. nur die Mädchen). Schnittwahrscheinlichkeiten lassen sich in der Vierfeldertafel direkt ablesen, während sie sich am Baumdiagramm nur über die Pfadmultiplikation ermitteln lassen. Einen richtigen Ansatz, der jedoch wieder verworfen wird, zeigen die Schülerinnen in (s58)-(s59): „Ich glaub', wir müssen das malrechnen“. Im Seminardiskurs kann hier auf das Prinzip der Darstellungsvernetzung hingewiesen werden: Möglicherweise hätten die Schülerinnen das ihrer Begründung zugrundeliegende Ergebnis in Frage gestellt, wenn sie (wie in der Aufgabe gefordert) weitere Darstellungen herangezogen hätten.

Ein zweiter auffälliger Punkt ist der starke Einfluss, den die Operatoren aus dem Aufgabentext auf die Aufgabenbearbeitung haben. Die Schülerinnen weichen vom eigentlich richtigen Weg zur Bestimmung der Schnittwahrscheinlichkeit durch Pfadmultiplikation $\mathrm{ab}$, weil sie sich uneins darüber sind, ob die involvierte Rechenoperation kompatibel mit dem Operator „begründe“ sei ((s58)-(s62)). Auch die Formulierungsversuche für den Antwortsatz weisen auf Schwierigkeiten im Zusammenhang mit den Operatoren hin. So kommen die Schülerinnen mehrmals auf die Frage zurück, ob es für eine Begründung ausreichend ist, wenn man auf das Ablesen von Werten aus einer Darstellung verweist (z. B. (s45); (s50)-(s51); (s76)-(s77)). Ähnliches zeigt sich in Bezug auf den Operator „bestimme“ in (s65 ff.).

Beide Aspekte können im Seminar zunächst zu einer vertieften Diskussion über den Zusammenhang von fachlichem und sprachlichem Lernen sowie Möglichkeiten der Sprachbildung im Unterricht führen. Zugleich bietet dieser Einstieg eine gute Grundlage für einen stärker forschungsbezogenen Zugang zum Transkript, wie in Arbeitsauftrag 2 vorgesehen.

\section{- Anmerkungen zum Arbeitsauftrag 2: Generieren von Forschungsfragen}

Im Seminarkontext lassen sich dann in der Auseinandersetzung mit dem empirischen Material Fragestellungen entwickeln. Hier einige Beispiele:

- Wie verwenden die Lernenden den Zufallsbegriff in ihren Argumentationen? (argumentationsbezogene Perspektive)

- Welches Verständnis haben die Lernenden von den genutzten Fachbegriffen? (individuelle Perspektive)

- Inwiefern nutzen die Lernenden spezifische Begriffe für die einzelnen Darstellungsformen sowie für den Wechsel zwischen den Darstellungsformen (Text - Diagramm - Einheitsquadrat)? (repräsentationsbezogene Perspektive) 
- Wie stellen die Lernenden den Zusammenhang zwischen absoluten Werten, relativen Häufigkeiten und Wahrscheinlichkeiten her und welche sprachlichen Mittel nutzen sie dafür? (stoffdidaktische Perspektive, Schwerpunkt Stochastik)

- Inwiefern werden die Lösungsversuche im Verlauf des gemeinsamen Denkprozesses sprachlich und fachlich differenzierter? (prozessorientierte Perspektive)

- An welchen Stellen zeigt sich ein sprachlicher oder fachlicher Klärungsbedarf und inwiefern wird er bearbeitet? (Problemlösungs-Perspektive)

Die Übung verdeutlicht den Studierenden exemplarisch, was anhand eines sehr kleinen Ausschnitts aus dem Handlungsfeld Schule alles untersucht werden kann. Im vorliegenden Beispiel lassen sich etwa theoretische Anknüpfungspunkte zu den Bereichen Begriffsbildung, Darstellungsvernetzung, Lernendenaktivierung, mathematisches Begründen oder individuelle Vorstellungsentwicklung herstellen. Auf forschungsmethodischer Ebene zeigt sich, dass ein interessantes Studienprojekt nicht notwendigerweise auf große Datenmengen oder komplexe Erhebungsmethoden angewiesen ist.

Die Frage, wie Studierende bei der Entwicklung eigener Forschungsfragen zum Thema Sprachbildung im Fach Mathematik systematisch unterstützt werden können, wird in - Abschn. 2.6 thematisiert.

\subsubsection{Ausschärfung der Projektidee}

Zum Zeitpunkt des zweiten Begleitseminars, etwa sechs Wochen nach Beginn des Praxissemesters an der Schule, haben die Studierenden eine vorläufige Projektskizze eingereicht. Ziel dieser Phase des Projekts ist es, die in der Skizze formulierte Projektidee weiter auszuschärfen, indem zum einen die Passung von Forschungsfrage, geplanter Erhebungsmethode und Probandenauswahl, zum anderen die Realisierbarkeit des Projekts mit Blick auf die Voraussetzungen an der Praktikumsschule überprüft wird. Dafür eignen sich die folgenden Übungen für den Seminarkontext.

\section{- Projektskizze schärfen I: „Speed-Dating“}

Die Studierenden bilden für diese Übung einen Innen- und einen Außenkreis. Im Innenkreis befinden sich die Studierenden, die ein Studienprojekt im Fach Mathematik durchführen, im Außenkreis die Kommilitonen ohne Studienprojekt; sie haben die Funktion von kollegialen Berater*innen. ${ }^{13}$ Die Studierenden im Innenkreis stellen ihr Studienprojekt in zwei Minuten ihrem Gegenüber vor und benennen dabei ihren fachlichen Schwerpunkt, die Forschungsfrage, den theoretischen Hintergrund, die geplante Forschungsmethode, das Projektszenario sowie Aspekte der Sprachbildung. Die Zeit ist bewusst äußerst knapp kalkuliert, damit die Studierenden sich auf wesentliche Aspekte ihres Vorhabens konzentrieren müssen. Der/die kollegiale Berater*in hat dann drei Minuten Zeit für Nachfragen. Nach Ablauf der fünf Minuten rücken die Studierenden im Außenkreis zwei Plätze weiter. Insgesamt werden drei Runden durchlaufen. In einer kurzen Rückmelde-Runde berichten die Studierenden, welche Erkenntnisse sie für ihr Studienprojekt gewonnen haben, wo sie in ihrer Weiterarbeit ansetzen möchten und wo sie Unterstützung benötigen.

13 Die Aufteilung der Studierenden in die beiden Gruppen kann natürlich variiert werden; es muss nur darauf geachtet werden, dass mindestens eine Studentin oder ein Student pro Paar auch tatsächlich eine Projektskizze zu diskutieren hat. 


\section{Arbeitsauftrag Speed-Dating an die Studierenden}

Stellen Sie Ihre Skizze mit Hilfe der folgenden Stichpunkte vor:

- Forschungsfrage

- Theoriehintergrund, Literatur

- Methode

- Konkretes Projektszenario (Zeitraum, Klasse, Design ....)

- Aspekte der Sprachbildung

Jede/r sollte anschließend in der Lage sein, seine/ihre Projektidee kurz und präzise zu formulieren.

Die Methode erweist sich als sehr wirksam, weil die Studierenden ihre Projektskizze innerhalb kürzester Zeit dreimal mündlich beschreiben müssen und sie Rückmeldungen aus drei verschiedenen Perspektiven bekommen. Gerade wenn die Nachfragen sich auf ähnliche Punkte beziehen (z. B. Forschungsmethode, Erhebungsdesign, Machbarkeit), erkennen die Studierenden, wo sie bei der Konkretisierung ihrer Projektskizze ansetzen können.

Auch Studierende, die ihr Studienprojekt nicht im Fach Mathematik durchführen, profitieren von dieser Übung, weil sie z. B. forschungsmethodologische Erkenntnisse auf die Planung ihrer Studienprojekte in anderen Fächern übertragen können.

Die Impulse aus dem Speed-Dating können direkt in die Weiterarbeit einfließen.

\section{- Projektskizze schärfen II: Weiterarbeit in Arbeitsgruppen}

Die anschließende Gruppenarbeit eignet sich, wenn eine erste Projektidee vorliegt und möglichst auch schon konkrete Fragen oder Probleme identifiziert wurden. Die Studierenden bilden für die Diskussion Gruppen à ca. vier Personen (plus reihum die Dozent*innen; Dauer ca. 45 min pro Studienprojekt). Sie erhalten folgenden Arbeitsauftrag:

\section{Arbeitsauftrag an die Studierenden zur Weiterarbeit in Arbeitsgruppen, Schwerpunkt Forschungsdesign}

Stellen Sie Ihr Studienprojekt in Ihrer Arbeitsgruppe kurz vor. Benennen Sie die Forschungsfrage und ein offenes Problem. Reflektieren Sie gemeinsam die Umsetzung des Projektes:

- Ist das Projekt reichhaltig?

- Passt die Methode zur Frage?

- Sind die für die Erhebung ggf. eingesetzten Aufgaben geeignet?

- Ist die Umsetzung realistisch?

Sammeln Sie Vorschläge zur Konkretisierung des Vorhabens. Welche Entscheidungen für die weitere Arbeit am Projekt ergeben sich?

Diskussionen in der Kleingruppe sind geeignet, um die in der Projektskizze angedachte Forschungsfrage zu schärfen und die geplante Datenerhebung zu besprechen. Zu den häu- 
fig diskutierten Aspekten gehören neben der Formulierung der Forschungsfrage (hierzu - Abschn. 2.6) das Finden geeigneter Erhebungsmethoden sowie das Design von Mathematikaufgaben, die der Datenerhebung dienen.

\subsubsection{Weiterarbeit am Erhebungsdesign}

Manche Studierende sind der Auffassung, dass sie große Datenmengen erheben müssen bzw. dass es leichter sei, große Datenmengen auszuwerten. Das Studienprojekt kann und soll aber nur einen kleinen Ausschnitt aus dem Handlungsfeld Schule beleuchten, und statistisch relevante Daten können ohnehin nicht oder nur schwerlich im Rahmen eines solchen Projektes erhoben werden. Die vorliegenden Projekte sind daher überwiegend qualitativ angelegt, die Analyse der eher wenigen Daten erfolgt hingegen umso genauer. Dabei spielt die Erhebungsmethode eine wichtige Rolle. So sind im Kontext des Seminars z. B. (geschlossene) Befragungen von Schüler*innen mit einem Fragebogen weniger geeignet, weil sich daran schülerseitige Lernprozesse bestenfalls indirekt untersuchen lassen. Für den hier beschriebenen Kontext besonders geeignet erscheinen daher vor allem Untersuchungen, die sich auf mündliche oder schriftliche Schülerprodukte beziehen.

Dass eine solche Erhebung zwar gut durchdacht, aber nicht umfangreich oder aufwändig sein muss, zeigen folgende Beispiele:

- Ein Studierender hat für sein Studienprojekt den Schülerinnen und Schülern einer 8. Klasse in einer Einführungsphase den Auftrag gegeben, die Begriffe „Wahrscheinlichkeit" und ,Zufall“ sowie den Zusammenhang zwischen beiden Begriffen schriftlich zu erklären (vgl. Abschn. 2.6.3.2).

- Eine Studentin hat Schüler*innen der 7. Klasse eine eigenständige schriftliche Ergebnissicherung zum Thema Mittelwerte mit Hilfe der SMS-Methode vornehmen lassen (vgl. Abschn. 2.2.2.2).

- Eine Studentin hat zwei Achtklässlerinnen eine Blütenaufgabe ${ }^{14}$ gemeinsam bearbeiten lassen und davon eine Audioaufnahme erstellt (vgl. $>$ Abschn. 3.1.1).

Diesen Erhebungen ist gemeinsam, dass die Studierenden mit minimalem Erhebungsaufwand Daten gewinnen konnten, die für das forschende Lernen sehr ergiebig sind und aus denen sich unterrichtspraktisch relevante Schlüsse ziehen lassen.

Wenn im Fach Mathematik mündliche oder schriftliche Schülerdaten erhoben werden, ist ein entscheidender Punkt das Aufgabendesign:

- Was genau sollen die Schüler*innen tun? Welche Aufgaben sollen sie bearbeiten und wie sollen diese konstruiert sein?

- Wie können sowohl mathematisch als auch sprachlich interessante Produkte entstehen?

- Wie kann gewährleistet werden, dass die Aufgaben für die Zielgruppe methodisch, fachlich und sprachlich angemessen sind?

- Wie kann das fachliche und sprachliche Vorwissen aktiviert werden - insbesondere dann, wenn sich die Aufgaben nicht unmittelbar auf die im Unterricht regulär bearbeiteten Inhalte beziehen?

Manchmal können die Studierenden an Aufgabenentwürfe aus dem Vorbereitungsseminar zum Praxissemester anknüpfen. Meistens ist aber eine Neukonzeption notwendig, um

14 Blütenaufgaben bestehen aus mehreren, zunehmend anspruchsvolleren Teilaufgaben zum selben Kontext (Bruder und Reibold 2012). Der Einstieg erfolgt über eine einfache, geschlossene Teilaufgabe, die weiteren Teilaufgaben können dann offener gestaltet sein. 
die Bedingungen im Praxissemester (Jahrgangsstufe, im Unterricht anstehende Inhalte, fachliche und sprachliche Kenntnisse der Schüler usw.) berücksichtigen zu können.

Mit Hilfe der Impulse aus der Arbeitsgruppe in der Lehrveranstaltung legen die Studierenden ihr endgültiges Erhebungsdesign fest. Die Mitstudierenden haben also eine wichtige Unterstützungsfunktion in einer entscheidenden Phase des Studienprojekts. Ebenso wichtig ist allerdings die Unterstützung durch die Lehrenden - gerade weil die Studierenden in ihren Projekten zwei Disziplinen gemeinsam denken müssen.

\subsubsection{Datenauswertung und Interpretation}

Die gleiche Arbeitsweise kann eingesetzt werden, wenn es um die Auswertung der an den Schulen erhobenen Daten geht. Auch hier ist die Unterstützung der Studierenden untereinander, aber auch durch die Lehrenden, von großer Bedeutung. Hilfreich erweist sich insbesondere zu Beginn die gemeinsame exemplarische Analyse im Plenum. Im Anschluss daran können die Studierenden dann in Gruppen weiterarbeiten, wobei ein möglicher Arbeitsauftrag lautet:

\section{Arbeitsauftrag an die Studierenden zur Weiterarbeit in Arbeitsgruppen, Schwerpunkt Datenauswertung}

Stellen Sie Ihre Daten und den Stand der Datenauswertung kurz vor. Benennen Sie offene Fragen, die Sie diskutieren möchten, und reflektieren Sie gemeinsam entlang der folgenden Punkte:

- Sollen alle Daten berücksichtigt werden oder nur eine Auswahl? Warum?

- Wie lassen sich die Daten systematisieren (z. B. bündeln, kontrastieren)?

- Aus welchen Perspektiven kann man die Daten analysieren? Welche theoretischen Konzepte unterstützen die Analyse?

- Wie können Daten unterschiedlichen Typs aufeinander bezogen werden (z. B. Schriftprodukte von Schüler*innen, zu denen die Schüler*innen interviewt werden)?

Dabei sollte insgesamt deutlich werden, dass sich mithilfe einer systematischen Analyse entlang der im Arbeitsauftrag genannten Aspekte auch aus einem kleinen oder vermeintlich wenig interessanten Datenkorpus wichtige Einsichten gewinnen lassen, die über das Studienprojekt hinaus für die Professionalisierung relevant sind. Oft betrifft dies auch die für die Erhebung gewählten Methoden: So erweisen sich für Studierende rückblickend gerade qualitative Methoden wie z. B. mündliche Befragungen von Schülern zu ihren Lösungsansätzen oder zu ihrem Aufgabenverständnis nicht nur als besonders aufschlussreich, sondern auch als gut auf die spätere Schulpraxis übertragbar und damit als gewinnbringend für die eigene Professionalisierung ( $\downarrow$ Abschn. 3.1).

\subsection{Forschungsfragen entwickeln}

॥ Dass die Mathematik mit ihren Textaufgaben auch für Muttersprachler sprachliche Hürden bereithält, kann ja niemand abstreiten. Aber wie gehen Lehrer damit um? Wie würde ich damit umgehen? Das war sozusagen meine Motivation, und daraus habe ich dann eine Forschungsfrage entwickelt (Studierender, Praxissemester 2018). 
Das Eingrenzen und Formulieren geeigneter Forschungsfragen ist eine wesentliche Aufgabe bei der Arbeit an einem wissenschaftlichen Projekt. Das gilt auch für kleinere studentische Untersuchungen wie Studienprojekte im Praxissemester. Denn Forschungsfragen bestimmen, unter welchem Gesichtspunkt wir uns einem Phänomen oder einer Problemstellung nähern; sie haben damit Auswirkungen auf das gesamte Forschungsdesign: Wie kann die Untersuchung theoretisch fundiert werden? Welche Art von Daten und wie viele Daten sollen erhoben werden, um die Frage beantworten zu können? Welche Erhebungsmethoden kommen dafür in Frage?

Für viele Studierende stellt die exakte Formulierung von Forschungsfragen eine große Herausforderung dar. Der vorliegende Abschnitt zeigt anhand von Praxisbeispielen,

- welche Kriterien Forschungsfragen im Kontext der Verbindung von Sprache und Fach erfüllen sollten,

- wie Studierende Ansatzpunkte für Forschungsfragen finden können,

- wie vorläufige Forschungsfragen präzisiert werden können.

Hinsichtlich der hochschuldidaktischen Design-Prinzipien wird in diesem Abschnitt die Integration von Sprache und Fach daher insbesondere auf der Ebene des forschenden Lernens (DP4) thematisiert.

\subsubsection{Vorläufige Forschungsfragen weiterentwickeln}

Grundlage jeder empirischen Untersuchung ist eine klar fokussierte und wissenschaftlich fundierte Forschungsfrage, die eine realistische Projektplanung ermöglicht. Dies gilt gleichermaßen für kleinere studentische Projekte z. B. im Rahmen einer Praxisphase. Für Studierende stellt das Finden einer geeigneten Forschungsfrage oft eines der zentralen Probleme im Praxissemester dar. So formulierte eine Studentin im Rückblick ( Abschn. 3.1):

I) Für mich war eines der wichtigsten Themen im Seminar, wie man eine Forschungsfrage findet. Damit tue ich mich furchtbar schwer. (...) Bei mir haben sich super viele Forschungsfragen aufgeworfen, die aber dann alle doch nicht Hand und Fuß hatten (Studentin, Praxissemester 2019).

Die Beispiele B1 und B2 zeigen vorläufige Forschungsfragen, die Studierende zu Beginn ihres Praxissemesters im Fach Mathematik mit Schwerpunkt Sprachbildung formuliert haben:

\section{Beispiel}

B1: „Inwieweit führt ein sprachsensibler Fachunterricht zu einem besseren Verständnis von Fachbegriffen (am Beispiel von ,Median', ,Arithmetisches Mittel' und ,Robustheit')?“

B2: „Welche Schwierigkeiten haben Schüler*innen der Jgst. 8 im Umgang mit Textaufgaben?“

Aufgabe im Rahmen der Lehrveranstaltung ist es, die vorläufigen Forschungsfragen der Studierenden gemeinsam zu überprüfen und kriteriengeleitet weiterzuentwickeln. ${ }^{15}$ Da-

15 Die Beispiele B1 und B2 werden am Ende von $>$ Abschn. 2.6 wieder aufgegriffen. 
bei besteht im Kontext einer sprachbewussten Hochschullehre eine besondere Anforderung darin, dass die Verknüpfung fachlicher und sprachlicher Aspekte bereits in der Forschungsfrage angelegt sein muss.

Anhand von Beispielen wie den oben genannten kann mit den Studierenden exemplarisch durchgespielt werden, wie Forschungsfragen präziser gefasst werden können. Dafür können die in Abschn. 2.6.2 vorgestellten Kriterien herangezogen werden.

\subsubsection{Kriterien für Forschungsfragen im Bereich Sprachbildung im Fach}

Die folgenden Kriterien können dabei unterstützen, vorläufige Forschungsfragen im hier diskutierten Kontext „Sprachbildung im Fach Mathematik“ zu prüfen und zu überarbeiten; sie lassen sich auch für andere Kontexte adaptieren.

\section{- Inwiefern steht die Forschungsfrage im Zusammenhang mit Sprachbildung im Fach} Mathematik?

Das erste Kriterium ist der interdisziplinären Ausrichtung im Kontext Sprache und Fach geschuldet: Die Forschungsfrage muss an der Schnittstelle von Mathematik und Sprachbildung verortet sein. Damit ist eine erste Bedingung und zugleich Möglichkeit zur Präzisierung des studentischen Vorhabens gegeben. So könnte eine Fragestellung in der Mathematikdidaktik ohne den Schwerpunkt Sprachbildung lauten: „Wie bearbeiten Lernende der Jgst. 8 kontextgebundene Aufgaben?“ Wird ein Schwerpunkt auf Sprachbildung gesetzt, so wäre eine mögliche Variante: „Wie interagieren Lernende der Jgst. 8 bei der Bearbeitung kontextgebundener Aufgaben in Partnerarbeit?"Im zweiten Fall ist also eine Fokussierung insofern gegeben, als die mündliche Kommunikation zwischen den Lernenden ausdrücklich zum Gegenstand der Analyse wird.

\section{- Welche Theoriebezüge zu den jeweiligen Disziplinen lassen sich herstellen?}

Gegenstand von studentischen Projekten im Kontext einer Praxisphase ist die systematische Untersuchung eines kleinen Ausschnitts aus dem Handlungsfeld Schule. Dabei bilden Beobachtungen aus der Schulpraxis häufig den Ausgangspunkt für die Entwicklung einer Forschungsfrage - insbesondere, wenn die Studierenden zu diesem Zeitpunkt bereits an einer Schule tätig sind. Um das Projekt von Vornherein wissenschaftlich zu fundieren, muss bei der Konzeption der Forschungsfrage geprüft werden, wie sich das Projekt theoretisch einbetten lässt. Dafür müssen Theoriebezüge zu den relevanten Disziplinen (Mathematikdidaktik, Linguistik, Sprach-/DaZ-Didaktik) hergestellt werden.

\section{- Ist die Forschungsfrage ausreichend eingegrenzt?}

Viele vorläufige Forschungsfragen sind zu breit angelegt, etwa wenn sie auf umfassende Ansätze (z. B. ,sprachsensibler Fachunterricht“) Bezug nehmen. Um ein Projekt sinnvoll bearbeiten zu können, ist die Fokussierung auf einen ausgewählten Teilaspekt (z. B. eine bestimmte Methode des sprachsensiblen Fachunterrichts) notwendig (vgl. unten die Diskussion des Beispiels B1). 


\section{- Ist die Forschungsfrage operationalisierbar?}

Manche Forschungsfragen beziehen sich auf abstrakte Konzepte wie „Motivation“, „Effizienz“ oder „Verständnis“, deren Erforschung komplexe Forschungsdesigns erfordern würden; sie können mit den verfügbaren fachlichen, zeitlichen und personellen Ressourcen nicht bearbeitet werden. Auch in diesem Fall muss die Forschungsfrage so adaptiert werden, dass sie im Rahmen des studentischen Vorhabens beantwortet werden kann. Dies lässt sich allerdings meist nicht mit einer einfachen Eingrenzung der Forschungsfrage lösen, sondern erfordert eine deutliche Modifikation oder Neukonzeption. Um an die ursprüngliche Idee, sich mit „Motivation“, „Effizienz“ oder „Verständnis“ auseinanderzusetzen, trotzdem anzuknüpfen, kann einleitend die Frage gestellt werden: „Woran erkenne ich, dass die Lernenden motiviert sind, dass die Methode effizient ist, dass das Verständnis der Lernenden sich verbessert hat?" Auf diese Weise können beobachtbare, überprüfbare Faktoren gesammelt werden, auf deren Grundlage eine neue Forschungsfrage entstehen kann.

\section{- Ist die Forschungsfrage mit Blick auf die Professionalisierung als zukünftige Lehrkraft relevant?}

Dieses Kriterium ist vor allem für studentische Projekte im Kontext von Praxisphasen von Bedeutung, wo neben dem wissenschaftlichen Erkenntnisgewinn vor allem die eigene Professionalisierung im Vordergrund steht:

॥ Im Zentrum steht damit der persönliche Erkenntnisgewinn. So zielt die intendierte TheoriePraxis-Verknüpfung vornehmlich darauf ab, die im Rahmen der Studienprojekte gewonnenen Erfahrungen und Erkenntnisse vor dem Hintergrund der eigenen Professionsentwicklung zu reflektieren (ZLB UDE 2019, S. 3).

Die Forschungsfrage führt im gelingenden Fall zu einem Vorhaben, bei dem die Studierenden zum einen ihre analytischen und methodischen Fähigkeiten bezüglich Sprache und Sprachbildung im Fach Mathematik (und darüber hinaus) stärken und bei dem sie zum anderen Forschungsmethoden kennenlernen, die sie später im Sinne der Lehrerhandlungsforschung anwenden können (z. B. um Schwierigkeiten von Schülerinnen und Schülern systematisch nachzugehen).

\subsubsection{Entwicklung von Forschungsfragen}

In Abschn. 2.5 wurde eine Übung vorgestellt, bei der das Gespräch zweier Schülerinnen bei der Bearbeitung einer Mathematikaufgabe aus unterschiedlichen forschungstheoretischen Perspektiven „,befragt“ wurde. Diese Übung hat veranschaulicht, dass man empirische Daten aus ganz unterschiedlichen Perspektiven betrachten kann und dass sich auch an begrenztem Datenmaterial unterschiedliche Fragestellungen entwickeln lassen. Allerdings ist die Vorgehensweise in einem empirischen Projekt in der Regel umgekehrt: Es werden zunächst Forschungsfragen formuliert, erst dann erfolgt die Datenerhebung. Zwar ist es nicht nur bei studentischen Vorhaben durchaus möglich und oft notwendig, die ursprüngliche Forschungsfrage anzupassen, wenn die Daten vorliegen (z. B. indem auf einen Teilaspekt fokussiert wird). Da aber die Forschungsfrage das gesamte Forschungsdesign maßgeblich beeinflusst, sollte sie bereits zu Projektbeginn sorgfältig durchdacht sein. 


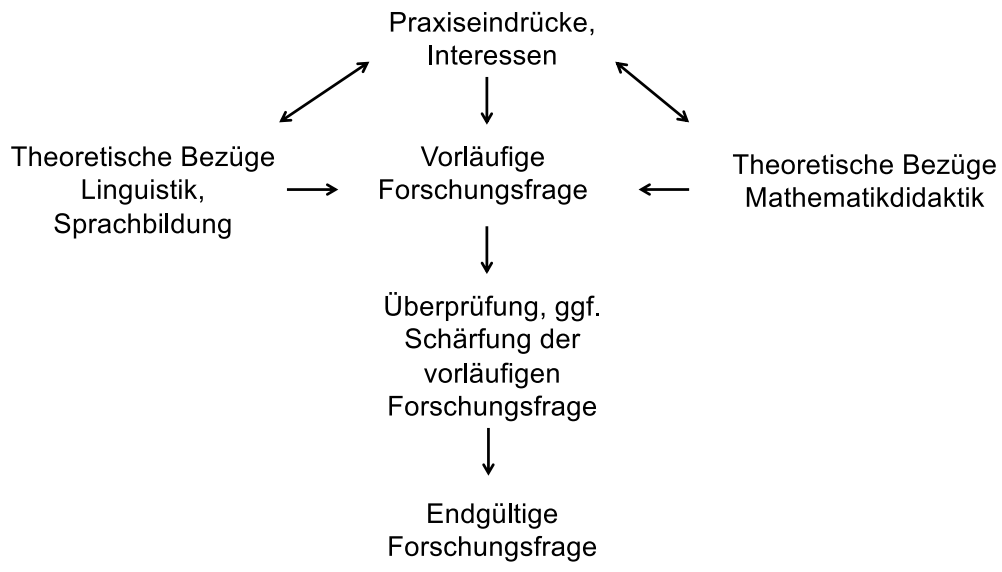

- Abb. 2.18 Entwicklung einer Forschungsfrage

Im Folgenden ( Abschn. 2.6.3.1-2.6.3.3) wird beschrieben, wie Forschungsfragen im Kontext des forschenden Lernens schrittweise entwickelt werden können. Den Ausgangspunkt bilden, wie in - Abb. 2.18 dargestellt, zum Beispiel Eindrücke aus der (Schul-)Praxis und Interessen der Studierenden. Unter Bezugnahme auf Literatur aus den Bereichen Mathematikdidaktik, Sprachbildung und Linguistik wird dann zunächst eine vorläufige Forschungsfrage formuliert. Diese wird (am besten kooperativ) überprüft und geschärft, bevor die endgültige Forschungsfrage festgelegt wird.

\subsubsection{Praxiseindrücke als Ausgangspunkt für erste Forschungsfragen}

I) Bei Aufgaben wie: „Lisa geht einkaufen, sie kauft drei Lutscher zu je achtzig Cent und zwei Flaschen Cola zu je einem Euro zehn“" wurde deutlich, dass schon einfache Präpositionen wie beispielsweise die Präposition ,je“ große Schwierigkeiten verursachen können, wenn sie übersehen werden. Aus diesem Grund habe ich dieses Projekt überhaupt begonnen. Eine Schülerin hatte dieses Wort übersehen, beziehungsweise sie wollte es übersehen, weil sie es nicht verstanden hat (Student, Praxissemester 2018).

Konkrete Probleme von Schülerinnen und Schülern, wie sie der Studierende im Umgang mit Textaufgaben beobachtet hat, stellen einen klassischen Ansatzpunkt für systematische Untersuchungen im Rahmen von Aktions-/Lehrerhandlungsforschung dar (Altrichter et al. 2018; Boeckmann et al. 2010; Klewin et al. 2017). Auch für studentische Projekte im Kontext des forschenden Lernens bietet es sich an, Erfahrungen oder Beobachtungen aus der Praxis aufzugreifen: Zum einen ist die Motivation hoch, wenn es darum geht, Lösungsansätze für eine praktische Frage zu entwickeln (oder zumindest das Problem besser zu verstehen); zum anderen sind aus der Praxis entspringende Forschungsideen mit konkreteren Vorstellungen verbunden als solche, die auf ein allgemeines Interesse (z. B. „Wie kann man die Motivation im Mathematikunterricht erhöhen?“) zurückgehen. Voraussetzung ist allerdings, dass die Studierenden das Handlungsfeld Schule bereits gut einschätzen können, sei es durch einen aktuellen Einblick in den schulischen Alltag in einer Praxisphase oder durch andere Erfahrungen mit Lernen- 
den (z. B. beim Nachhilfeunterricht, bei der Unterstützung geflüchteter Schüler*innen, bei der Hausaufgabenbetreuung im Hort usw.). Einige Beispiele:

- Ein kleines Wort wie die Präposition ,je“ kann dazu führen, dass eine Textaufgabe falsch bearbeitet wird.

- Viele Lernende in der Klasse verwechseln „bedingte Wahrscheinlichkeit“ und „Schnittwahrscheinlichkeit“.

- Die beiden neu zugewanderten Schüler können gut rechnen, sie können aber ihre Lösungswege nicht gut erklären.

- Die Merksätze im Mathebuch sind schwer verständlich.

Solche Beobachtungen können den Kern für eine dann genauer zu spezifizierende und theoretisch zu begründende Forschungsfrage bilden.

\subsubsection{Theoretische Fundierung von Studienprojekten}

॥ Es ist eine Kernfrage der Lehrerbildung, wie Wissen, das in der universitären Ausbildungsphase angehender Lehrerinnen und Lehrer erworben wird, in der Praxis zum Tragen kommt. Im Hinblick auf die in Schule vorherrschende Alltagsbelastung, bei der Handlungsmodifikationen nicht allein durch abstraktes Wissen herbeizuführen sind, bieten Praxisphasen im Studium die Möglichkeit, das an der Universität Gelernte mit der Reflexion des eigenen erfahrungsbasierten Kompetenzerwerbs in der Schule zu verknüpfen (v. Ackeren und Herzig 2016, S. 4).

Das Praxissemester und insbesondere die Studienprojekte bieten für die Studierenden eine sinnstiftende Möglichkeit, um universitäre Inhalte mit schulischer Praxis in Beziehung zu setzen. Die theoriegeleitete Untersuchung und Einordnung von Praxiseindrücken soll zur Professionalisierung beitragen und die zukünftigen Lehrkräfte befähigen, ,nicht spontan zu agieren, sondern Erfahrungen [zu] ordnen und [zu] lernen, mit künftigen und unbestimmten Situationen umgehen zu können.“ (v. Ackeren und Herzig 2016, S. 4)

Wichtige theoretische Bezugspunkte für Studienprojekte bilden zunächst die Konzepte aus der Fachdidaktik des studierten Lehramtsfachs, hier also aus der Mathematikdidaktik. Dazu gehören zum Beispiel:

- Konstruktion und Variation mathematischer Lernumgebungen

- mathematische Darstellungsformen und Darstellungsvernetzung

- individuelle Vorstellungen, Vorstellungsentwicklung und mathematische Begriffsbildung

- Phasierung mathematischer Lern- und Lehrprozesse (Kernprozesse Entdecken, Ordnen, Vertiefen)

- Nutzung digitaler Werkzeuge

- Unterscheidung von Lern-, Übungs- und Prüfungsaufgaben

- Binnendifferenzierung

Durch die gleichzeitige Berücksichtigung des Themas Sprachbildung erweitert sich die theoretische Basis um Lerninhalte aus den Bereichen Linguistik und Sprachdidaktik, etwa:

- Alltags- und Fachsprache

- Mündlichkeit und Schriftlichkeit

- linguistische Kategorien zur Beschreibung von Fachkommunikation 
- Lernersprachenanalyse; Fehleranalyse

- Ansätze für sprachbewussten Fachunterricht (z. B. Scaffolding, genredidaktische Zugänge, Methoden des sprachsensiblen Unterrichts)

- Sprachvergleich

- mehrsprachiges Handeln (z. B. Translanguaging, Code-Switching)

Nicht alle der genannten Themen können in den Vorbereitungs- und Begleitseminaren ausführlich behandelt werden; die Studierenden sollten daher auch auf ihr Wissen aus vorangegangenen oder parallel verlaufenden Veranstaltungen zurückgreifen bzw. sich selbst mit neuen thematischen Bereichen vertraut machen.

Anhand der Forschungsfrage eines Studierenden im Praxissemester 2019 lässt sich aufzeigen, wie theoretische Bezüge hergestellt werden können. Die Forschungsfrage lautete:

॥) Wie erklären Schüler*innen der Jahrgangsstufe 8 die mathematischen Fachbegriffe „Wahrscheinlichkeit“" und „Zufall“?

Der Studierende ließ die Schülerinnen und Schüler seiner 8. Klasse die Fachbegriffe „Wahrscheinlichkeit“ und „Zufall“ in einer Einführungsphase schriftlich erklären; die Begriffe waren also noch nicht eingeführt worden. Die Schüler*innen schrieben sehr kurze Texte wie die in $\bullet$ Abb. 2.19 aufgeführten.

Die meisten der hier abgedruckten Beispiele lassen auf ein alltägliches Begriffsverständnis schließen - „Wahrscheinlichkeit“ wird mit „,hoher Wahrscheinlichkeit“ gleichgesetzt (z. B. ,was schon fast Perfekt ist in \%“; ,,das was zu $75 \%$ passiert“; ,wenn etwas wahrscheinlich passiert"). Einige Schüler*innen knüpfen an konkrete Alltagserfahrungen (und die damit verbundenen Hoffnungen einer hohen Gewinnwahrscheinlichkeit) an, etwa die Fußballnachrichten oder das Würfelspiel. Ein neutraler Zugang zeigt sich demgegenüber im letzten Beispiel (,wenn etwas passieren kann aber nicht muss“). Manche Schülerprodukte (wie oben das Beispiel ,wenn z.b.s ein würfel würfelt"“) zielen wiederum eher auf den Zufallsbegriff ab.

Diese und weitere Schülertexte untersuchte der Studierende im Hinblick auf individuelle Vorstellungen unter Bezug auf verschiedene Wahrscheinlichkeitsmodelle (Krüger et al. 2015; Laakmann und Schnell 2015), die Nutzung verschiedener Darstellungsformen (Prediger et al. 2011; Wessel 2015) sowie allägliches und mathematisches Begriffsverständnis (Weinrich 1989; George 2015; Guckelsberger und Schacht 2018). Die gewählte Forschungsfrage ermöglicht also vielfältige Theoriebezüge für eine fach- und sprachintegrierte Untersuchung.

- Abb. 2.19 Schülertexte zum Begriff „Wahrscheinlichkeit“"
Wahrscheinlichkeit:

- Was fast schon Perfekt ist in \% (Fussball News)

- das was zu 75\% passiert

- Eine Wahrscheinlichkeit ist wenn etwas wahrscheinlich passiert

_ ist wenn z.b.s ein würfel würfelt und hofft das es eine 6 wird

- Wenn man was weiß aber sich nicht sicher ist.

- Wenn etwas passieren kann aber nicht muss 


\subsubsection{Mit Forschungsfragen arbeiten}

Sobald eine vorläufige Forschungsfrage vorliegt, muss diese sorgfältig geprüft und ggf. überarbeitet werden. Um dies zu veranschaulichen, greifen wir die eingangs zitierten vorläufigen Forschungsfragen B1 und B2 auf.

\section{Kommentar zur vorläufigen Forschungsfrage B1}

B1: „Inwieweit führt ein sprachsensibler Fachunterricht zu einem besseren Verständnis von Fachbegriffen (am Beispiel von ,Median“, ,Arithmetisches Mittel“ und ,Robustheit')?“" (vorläufige Forschungsfrage, Praxissemester 2018)

Die Idee der Studentin ist zwar grundsätzlich interessant und für den Kontext „Sprachbildung im Fach Mathematik" relevant; auch die Fachbegriffe, um deren Verständnis es geht, werden konkret benannt. Es stellen sich aber mindestens die folgenden Fragen:

- Welche der vielfältigen Methoden und Ansätze im Bereich des sprachsensiblen Fachunterrichts sollen angewandt werden?

- Woran wird festgemacht, ob bzw. inwieweit sich das Verständnis der Schüler*innen verbessert?

- Lässt sich „Verständnis“ überhaupt (direkt) überprüfen? ${ }^{16}$

Wichtig ist an dieser Stelle also, Klarheit über das Erkenntnisinteresse zu gewinnen und zugleich die Realisierbarkeit im Rahmen der begrenzten Möglichkeiten des Studienprojekts kritisch zu prüfen. Im vorliegenden Fall hat die Studierende ihre Forschungsfrage folgendermaßen weiterentwickelt:

B1': „Inwieweit können Schüler*innen der Jahrgangsstufe 8 den Unterschied zwischen Median und arithmetischem Mittel schriftlich erklären?“

Durch diese Veränderung wird das Vorhaben einerseits spezifischer, andererseits eröffnen sich neue theoretische Bezugspunkte. Der ursprünglich genannte Aspekt des sprachsensiblen Fachunterrichts spiegelt sich in der Frage nur noch indirekt, wurde aber inhaltlich konkretisiert: Die Schülerinnen und Schüler sollen eine Erklärung schreiben. Die Studierende knüpft damit an Empfehlungen zur Integration von Schreibaufgaben ${ }^{17}$ in den Fachunterricht an. Ebenso lassen sich mathematikdidaktische Bezüge herstellen - etwa zur Diskussion um das diagnostische Potential von Schülertexten im Fach Mathematik. ${ }^{18}$

16 Vergleichbares merken Klewin et al. (2017, S. 147 f.) für Konzepte wie „Motivation“ oder „Stimmung“ an: „Manche Forschungsfragestellungen sind der Beobachtung schwer zugänglich, z. B.: „Ist die Motivation der SchülerInnen größer beim Frontalunterricht oder bei offenen Unterrichtsformen?“ oder „Wie ist die Stimmung in Klasse X“? Motivation lässt sich nicht direkt für die Beobachtung operationalisieren, sondern ist immer schon ein Ergebnis der Interpretation beobachtbarer Variablen. Stimmung lässt sich schlecht messen."

17 Leisen (2010), Gogolin und Lange (2010), Schmölzer-Eibinger et al. (2013), Schmölzer-Eibinger und Thürmann (2015) sowie Leitidee L2.

18 Götze (2019), Sjuts (2007). 
Über die Analyse der schriftlichen Lernendenprodukte lassen sich konkrete Aussagen sowohl über sprachliche als auch über fachliche Kenntnisse der Lernenden treffen. Die so gewonnenen Einsichten könnten dann wiederum genutzt werden, um ein Konzept für fach- und sprachintegriertes Lernen zu entwickeln. Die schulpraktische Relevanz der Forschungsfrage lässt sich nicht zuletzt über die im Mathematiklehrplan aufgeführten prozessbezogenen Kompetenzen „Kommunizieren“ und „Argumentieren“ herstellen.

\section{Kommentar zur vorläufigen Forschungsfrage B2}

B2: „Welche Schwierigkeiten haben Schüler*innen der Jgst. 8 im Umgang mit Textaufgaben?"

Viele Studierende neigen dazu, in ihren vorläufigen Forschungsfragen Probleme von Lernenden in den Vordergrund zu stellen. Typische Formulierungsmuster sind dann etwa:

- „Welche Probleme treten bei X auf?“

- „Welche Fehler zeigen Schüler*innen bei X?“

- „Welche Schwierigkeiten haben Schüler*innen mit X?“

- „Inwiefern sind Hürden bei X auf Y zurückzuführen?“

In der Schulpraxis fallen die Dinge, die nicht gelingen oder nicht verstanden werden, oft besonders ins Auge. Es ist daher gut nachvollziehbar, dass viele Studierende in ihren vorläufigen Forschungsfragen und Projektideen ein „Problem“ genauer untersuchen möchten. Die Problem- oder Defizitorientierung kann jedoch den Blick darauf verstellen, über welche mathematischen und sprachlichen Kompetenzen die Lernenden bereits verfügen und woran individuell oder im Klassenverband angeknüpft werden kann. Auch ist zu befürchten, dass am Ende des Studienprojekts eine gewisse Unzufriedenheit zurückbleibt, wenn aus der Literatur bekannte Probleme bei den Lernenden zwar erneut bestätigt, nicht aber produktiv bearbeitet wurden. So könnte ein fiktives Szenario zur Bearbeitung der Forschungsfrage B2 folgendermaßen aussehen:

\section{Fiktives Szenario zur Bearbeitung der Forschungsfrage B2}

B2: „Welche Schwierigkeiten haben Schüler*innen der Jgst. 8 im Umgang mit Textaufgaben?"

Fiktives Szenario: Der Studierende lässt Lernende der Jgst. 8 eine mathematische Textaufgabe schriftlich bearbeiten. Die Schülerlösungen untersucht er unter Bezug auf Fachliteratur nach mathematischen und sprachlichen Fehlern. Es zeigt sich, dass die in der Literatur diskutierten ,sprachlichen Hürden“ von Textaufgaben sich auch in den gesammelten Daten spiegeln. 
Der Studierende hätte in diesem Szenario eine Bestätigung für bereits bekannte Problembereiche bekommen, aber keine Erkenntnisse für die Unterrichtsgestaltung oder die individuelle Förderung von Lernenden gewonnen. Das Vorhaben hätte also vergleichsweise wenig zu seiner Professionalisierung beigetragen.

Es sollte daher grundsätzlich eine stärker phänomenbezogene Sichtweise eingenommen werden, bei der z. B. Lernendenbeiträge theoriegeleitet untersucht und verstehend nachvollzogen werden. Eine neutral formulierte Forschungsfrage unterstützt eine solche Vorgehensweise und lenkt den Blick auf konkrete Optionen für das unterrichtliche Handeln (z. B. Aufgabenvariation; Wissensspeicher statt Merksätzen; Darstellungen als Unterstützung).

Der Studierende hat seine vorläufige Forschungsfrage B2 überarbeitet; die endgültige Forschungsfrage B2' lautet:

B2': „Inwiefern weichen die Mathematikleistungen von Schüler*innen der Jgst. 8 bei Textaufgaben voneinander ab, wenn die sprachlichen Anforderungen größer und kleiner werden?“

Das Projekt wurde dann folgendermaßen umgesetzt:

\section{Verlauf des Studienprojekts zur Forschungsfrage B2'}

Im Studienprojekt bezieht der Studierende sich auf wissenschaftliche Erkenntnisse a) zur Lösungshäufigkeit von numerischen Aufgaben im Unterschied zu Textaufgaben sowie b) zu sprachlichen Hürden in Textaufgaben. Er konstruiert vier Aufgaben in jeweils drei Varianten: Variante 1 ist eine rein numerische Aufgabe, Variante 2 ist eine durchschnittlich komplex formulierte Textaufgabe, Variante 3 ist eine gezielt sprachlich entlastete Textaufgabe. Er lässt die Schüler*innen die drei Aufgabenvarianten bearbeiten; ergänzend führt er einige Schülerinterviews dazu durch. Die Ergebnisse geben einerseits Aufschluss darüber, was den Schüler*innen bei der Aufgabenbearbeitung hilft, andererseits, wo eine gezielte (fach-)sprachliche Unterstützung notwendig ist (z. B. Präpositionen im Fachkontext).

Die Feststellung, dass bestimmte sprachliche Eigenschaften von Textaufgaben die Mathematikleistungen beeinflussen, ist im tatsächlich durchgeführten Projekt also nicht mehr Ziel, sondern Voraussetzung für die Untersuchung. Im Projekt werden wissenschaftliche Erkenntnisse aufgegriffen und produktiv für eine bewusste Aufgabenvariation genutzt. Darüber hinaus werden einzelne Lernende genauer zu ihren Aufgabenbearbeitungen befragt. Das Studienprojekt trägt also aus fach- und sprachdidaktischer als auch aus forschungsmethodischer Sicht zur Professionalisierung bei. 


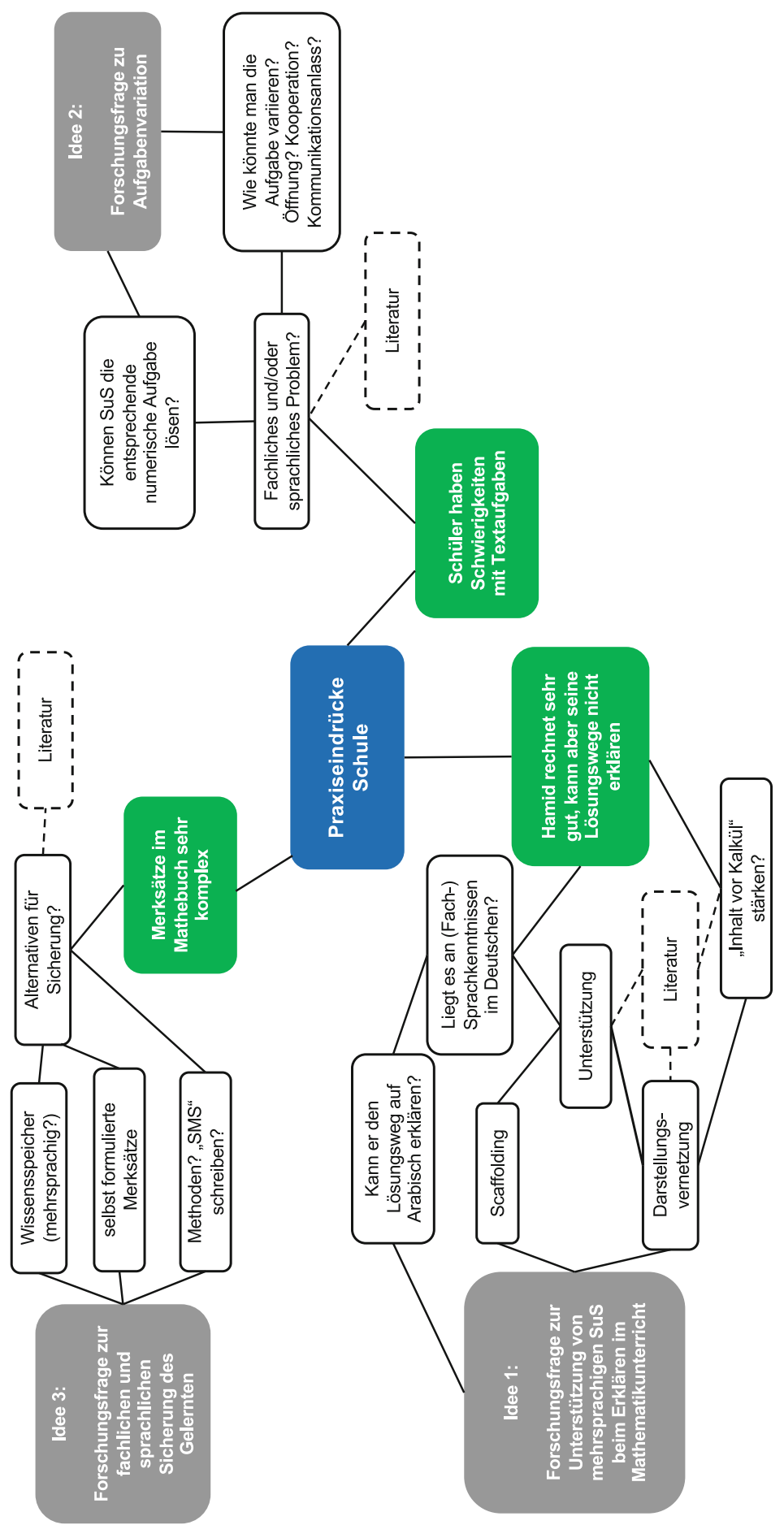

- Abb. 2.20 Mindmap zur Entwicklung einer Forschungsfrage 
Abschließend sei eine Brainstorming-Übung vorgeschlagen, die Studierende bei der Findung und Weiterentwicklung von Forschungsfragen im Kontext einer Praxisphase unterstützt:

\section{Arbeitsauftrag Brainstorming an die Studierenden}

1) Beginnen Sie eine Mindmap mit Beobachtungen, die in Verbindung mit dem Thema „Sprachbildung im Mathematikunterricht“ stehen. Greifen Sie dabei auf Ihre Notizen zu eigenem Unterricht oder zu Unterrichtsbeobachtungen zurück. Zum Beispiel: „Viele Schüler verstehen die Textaufgaben nicht.“

2) Wählen Sie dann zwei Beobachtungen aus und entwickeln Sie sie systematisch weiter:

- Welche Fragen oder Hypothesen haben Sie zu den Beobachtungen?

- Welche Verbindungen zu theoretischen Konzepten aus dem Studium sehen Sie?

- Wo können Sie eine erste Spezifizierung vornehmen (z. B. Fokus auf eine Zielgruppe, einen Lerninhalt, eine bestimmte Fachtextsorte ...)?

- Fügen Sie erste Literaturverweise ein.

- Machen Sie sich Notizen für weitere Recherchen.

3) Entwickeln Sie Ihre Mindmap im Austausch mit Kommiliton*innen weiter.

- Abb. 2.20 zeigt eine exemplarische Mindmap, wie sie im Rahmen von Lehrveranstaltungen entstehen kann. Den Ausgangspunkt bilden Praxiseindrücke, die die Studierenden an der Schule sammeln. Diese werden zum einen um Fragen und Hypothesen erweitert, zum anderen werden Bezüge zu Inhalten aus universitären Lehrveranstaltungen im Bereich Mathematikdidaktik und Sprachbildung hergestellt.

Die zunächst individuell erstellte Mindmap wird im Austausch mit den Mitstudierenden sukzessive ergänzt und ausdifferenziert. Sie bildet zudem eine gute Grundlage für Beratungsgespräche, weil sich nachvollziehen lässt, welche Zusammenhänge die Studierenden herstellen.

Open Access Dieses Kapitel wird unter der Creative Commons Namensnennung 4.0 International Lizenz (http://creativecommons.org/licenses/by/4.0/deed.de) veröffentlicht, welche die Nutzung, Vervielfältigung, Bearbeitung, Verbreitung und Wiedergabe in jeglichem Medium und Format erlaubt, sofern Sie den/die ursprünglichen Autor(en) und die Quelle ordnungsgemäß nennen, einen Link zur Creative Commons Lizenz beifügen und angeben, ob Änderungen vorgenommen wurden.

Die in diesem Kapitel enthaltenen Bilder und sonstiges Drittmaterial unterliegen ebenfalls der genannten Creative Commons Lizenz, sofern sich aus der Abbildungslegende nichts anderes ergibt. Sofern das betreffende Material nicht unter der genannten Creative Commons Lizenz steht und die betreffende Handlung nicht nach gesetzlichen Vorschriften erlaubt ist, ist für die oben aufgeführten Weiterverwendungen des Materials die Einwilligung des jeweiligen Rechteinhabers einzuholen. 